\title{
Pion-pion scattering amplitude
}

\author{
J. R. Peláez \\ Departamento de Física Teórica, II (Métodos Matemáticos), Facultad de Ciencias Físicas, Universidad Complutense de Madrid, \\ E-28040, Madrid, Spain \\ F. J. Ynduráin \\ Departamento de Física Teórica, C-XI Universidad Autónoma de Madrid, Canto Blanco, E-28049, Madrid, Spain
}

(Received 3 December 2004; published 18 April 2005)

\begin{abstract}
We obtain reliable $\pi \pi$ scattering amplitudes consistent with experimental data, both at low and high energies, and fulfilling appropriate analyticity properties. We do this by first fitting experimental low energy $\left(s^{1 / 2} \leq 1.42 \mathrm{GeV}\right)$ phase shifts and inelasticities with expressions that incorporate analyticity and unitarity. In particular, for the $\mathrm{S}$ wave with isospin 0 , we discuss in detail several sets of experimental data. This provides low energy partial wave amplitudes that summarize the known experimental information. Then, we impose Regge behavior as follows from factorization and experimental data for the imaginary parts of the scattering amplitudes at higher energy, and check fulfillment of dispersion relations up to $0.925 \mathrm{GeV}$. This allows us to improve our fits. The ensuing $\pi \pi$ scattering amplitudes are then shown to verify dispersion relations up to $1.42 \mathrm{GeV}$, as well as $s-t-u$ crossing sum rules and other consistency conditions. The improved parametrizations therefore provide a reliable representation of pion-pion amplitudes with which one can test chiral perturbation theory calculations, pionium decays, or use as input for $C P$-violating $K$ decays. In this respect, we find $\left[a_{0}^{(0)}-a_{0}^{(2)}\right]^{2}=(0.077 \pm 0.008) M_{\pi}^{-2}$ and $\delta_{0}^{(0)}\left(m_{K}^{2}\right)-\delta_{0}^{(2)}\left(m_{K}^{2}\right)=52.9 \pm 1.6^{\circ}$.
\end{abstract}

DOI: 10.1103/PhysRevD.71.074016

PACS numbers: 13.75.Lb, 11.55.Jy, 11.80.Et, 12.39.Fe

\section{INTRODUCTION}

A precise and unbiased knowledge of the $\pi \pi$ scattering amplitude has become increasingly important in recent years. This is so, in particular, because $\pi \pi$ scattering is one of the few places where one has more observables than unknown constants in a chiral perturbation theory (ch.p.t.) analysis to one loop, so it provides a test of ch.p.t. in this approximation, as well as a window to higher order terms. Beside this, an accurate determination of the $S$ wave scattering lengths, and of the phase shifts, provides essential information for three subjects under intensive experimental investigation at present, viz., light scalar spectroscopy, pionic atom decays, and $C P$ violation in the kaonic system.

In two recent papers, Ananthanarayan, Colangelo, Gasser, and Leutwyler [1] and Colangelo, Gasser, and Leutwyler [2] have used experimental information, analyticity and unitarity (in the form of the Roy equations [3]) and, in the second paper, also chiral perturbation theory, to construct a precise $\pi \pi$ scattering amplitude at low energy, $s^{1 / 2} \leq 0.8 \mathrm{GeV}$. Unfortunately, however, the analysis of Refs. [1,2] presents some weak points. First of all, the input scattering amplitude at high energy $\left(s^{1 / 2} \gtrsim 1.5 \mathrm{GeV}\right)$ which these authors use is incompatible with experimental data [4] on $\pi \pi$ cross sections and also contradicts known properties of standard Regge theory; see a detailed analysis in Refs. [5-7] and Appendix B here. Moreover, the errors these authors take for some of their experimental input data are optimistic, as shown in Ref. [7]. As a consequence of all this the $\pi \pi$ amplitudes of Ref. [2] do not satisfy well a number of consistency tests, as we show in Sec. VII here (see also Refs. [6,7] for more details).

Some of the shortcomings of the articles in Refs. [1,2], notably incorrect Regge behavior, are also reproduced in the papers of Descotes et al., and Kamiński, Leśniak, and Loiseau [8], who also base their analysis in the Roy equations but, since the errors given by these authors are substantially larger than those of Ref. [2], their effects are now less pronounced. Therefore, we still need to find reliable pion-pion scattering amplitudes compatible with physical data both at high and low energy, as well as to verify to what extent such amplitudes agree with ch.p.t.

In the present paper we address ourselves to the first question; that is to say, we try to find what experiment implies for the $\pi \pi$ amplitudes. To avoid biases, we will start by performing fits to experimental data on phase shifts and inelasticities, incorporating only the highly safe requirements of analyticity and unitarity, in the low energy region $s^{1 / 2} \leq 1.42 \mathrm{GeV}$. In particular, for the S0 wave below $0.95 \mathrm{GeV}$, where the experimental situation is confused, we perform a global fit, as well as individual fits to various sets of data. These fits are described in Sec. II and, in the following sections, we investigate to which extent the ensuing scattering amplitudes are consistent, in particular, with high energy information. To do so, we assume Regge behavior, as given in Ref. [5] (slightly improved; see Appendix B), above $1.42 \mathrm{GeV}$ : using this we check in Sec. III fulfillment of forward dispersion relations, for the three independent $\pi \pi$ scattering amplitudes. This, in particular, permits us to discriminate among the various 
sets of phase shifts for the S0 wave, leaving only a few solutions which are consistent with dispersion relations (and, as it turns out, very similar one to the other, as discussed in Sec. IV).

When dealing with different data sets one has to weigh not only the data on a single experiment but one has to take into account the reliability of the experiments themselves. So we have done for many waves, where some clearly faulty experimental data have only been considered to conservatively enlarge the uncertainties. Concerning the most controversial S0 wave, we have used the very reliable data coming from $K_{l 4}$ and $K \rightarrow \pi \pi$ decays; to this we add the results from other experimental analyses of $\pi \pi$ scattering available in the literature, either separately or combined in a global fit. We then use forward dispersion relations to test consistency of the several sets of data.

The present study should therefore be considered, in particular, as a guideline to the consistency (especially with forward dispersion relations) of the various data sets.

Next, we use these dispersion relations to improve the central values of the parameters of the fits given in Sec. II. The result of such analysis (Sec. IV) is that one can get a precise description for all waves, consistent with forward dispersion relations up to $s^{1 / 2} \sim 0.95 \mathrm{GeV}$ and a bit less so $\left(\lesssim 1.5 \sigma\right.$ level) in the whole energy range, $2 M_{\pi} \leq s^{1 / 2} \leq$ $1.42 \mathrm{GeV}$, and even below threshold, down to $s^{1 / 2}=$ $\sqrt{2} M_{\pi}$. The greater uncertainties affect the S0 wave for $s^{1 / 2}>0.95 \mathrm{GeV}$, a not unexpected feature, and, to a lesser extent, the $\mathrm{P}$ wave above $1.15 \mathrm{GeV}$.

In Sec. $\mathrm{V}$ we verify that the scattering amplitudes we have obtained, which were shown to satisfy $s-u$ crossing (by checking the dispersion relations), also verify $s-t$ crossing, in that they satisfy two typical crossing sum rules. In Sec. VI we use the scattering amplitudes we have determined and the method of the Froissart-Gribov representation to calculate a number of low energy parameters for P, D and some higher waves which, in particular, provides further consistency tests. We also evaluate, in Sec. VII, the important quantities $\left[a_{0}^{(0)}-a_{0}^{(2)}\right]^{2}$ and $\delta_{0}^{(0)}\left(m_{K}^{2}\right)-\delta_{0}^{(2)}\left(m_{K}^{2}\right)$ for which we find

$$
\begin{aligned}
{\left[a_{0}^{(0)}-a_{0}^{(2)}\right]^{2} } & =(0.077 \pm 0.008) M_{\pi}^{-2}, \\
\delta_{0}^{(0)}\left(m_{K}^{2}\right)-\delta_{0}^{(2)}\left(m_{K}^{2}\right) & =52.9 \pm 1.6^{\circ} .
\end{aligned}
$$

Also in Sec. VII we compare our results with those obtained by other authors using Roy equations and ch.p.t. However, in the present paper we will not address ourselves to the question of the chiral perturbation theory analysis of our $\pi \pi$ amplitudes.

Our paper is finished in Sec. VIII with a brief summary, as well as with a few appendixes. In Appendix A, we collect the formulas obtained with our best fits. In Appendix B we give a brief discussion of the Regge formulas used; in particular, we present an improved evaluation of the parameters for rho exchange. Appendix $\mathrm{C}$ is devoted to a discussion of the shortcomings of experimental phase shift analyses above $\sim 1.4 \mathrm{GeV}$, which justifies our preference for using Regge formulas in this energy region.

We end this introduction with a few words on notation and normalization conventions. We will here denote amplitudes with a fixed value of isospin, say $I$, in channel $s$, simply by $F^{(I)}, f_{l}^{(I)}$; we will specify the channel, $F^{\left(I_{s}\right)}$, when there is danger of confusion. For amplitudes with fixed isospin in channel $t$, we write explicitly $F^{\left(I_{t}\right)}$.

For scattering amplitudes with well-defined isospin in channel $s, I_{s}$, we write

$$
\begin{aligned}
F^{\left(I_{s}\right)}(s, t) & =2 \times \sum_{l=\text { even }}(2 l+1) P_{l}(\cos \theta) f_{l}^{\left(I_{s}\right)}(s), \quad I_{s}=\text { even, } \\
F^{\left(I_{s}\right)}(s, t) & =2 \times \sum_{l=\text { odd }}(2 l+1) P_{l}(\cos \theta) f_{l}^{\left(I_{s}\right)}(s), \quad I_{s}=\text { odd }, \\
f_{l}^{(I)}(s) & =\frac{2 s^{1 / 2}}{\pi k} \hat{f}_{l}^{(I)}, \quad \hat{f}_{l}^{(I)}=\sin \delta_{l}^{(I)}(s) e^{i \delta_{l}^{(I)}(s)} .
\end{aligned}
$$

The last formula is only valid when only the elastic channel is open. When inelastic channels open this equation is no more valid, but one can still write

$$
\hat{f}_{l}(s)=\left[\frac{\eta_{l} e^{2 i \delta_{l}}-1}{2 i}\right] .
$$

The factor 2 in the first formulas in (1.1a) is due to Bose statistics. Because of this, even waves only exist with isospin $I=0,2$ and odd waves must necessarily have isospin $I=1$. For this reason, we will often omit the isospin index for odd waves, writing e.g. $f_{1}, f_{3}$ instead of $f_{1}^{(1)}, f_{3}^{(1)}$. Another convenient simplification that we use here is to denote the $\pi \pi$ partial waves by S0, S2, P, D0, D2, $F$, etc., in self-explanatory notation.

The quantity $\eta_{l}$, called the inelasticity parameter for wave $l$, is positive and smaller than or equal to unity. The elastic and inelastic cross sections, for a given wave, are given in terms of $\delta_{l}$ and $\eta_{l}$ by

$$
\sigma_{l}^{\mathrm{el}}=\frac{1}{2}\left\{\frac{1+\eta_{l}^{2}}{2}-\eta \cos 2 \delta_{l}\right\}, \quad \sigma_{l}^{\mathrm{inel}}=\frac{1-\eta_{l}^{2}}{4} ;
$$

$\sigma_{l}^{\mathrm{el}}, \sigma_{l}^{\text {inel }}$ are defined so that, for collision of particles $A, B$ (assumed distinguishable),

$$
\sigma_{\text {tot }}=\frac{4 \pi^{2}}{\lambda^{1 / 2}\left(s, m_{A}, m_{B}\right)} \frac{2 s^{1 / 2}}{\pi k} \sum_{l}(2 l+1)\left[\sigma_{l}^{\mathrm{el}}+\sigma_{l}^{\mathrm{inel}}\right] .
$$

When neglecting isospin violations (which we do unless explicitly stated otherwise) we will take the convention of approximating the pion mass by $M_{\pi}=m_{\pi^{ \pm}} \simeq$ 139.57 MeV. We also define scattering lengths, $a_{l}^{(I)}$, and 
effective range parameters, $b_{l}^{(I)}$, by

$$
\begin{gathered}
\frac{\pi}{4 M_{\pi} k^{2 l}} \operatorname{Re} f_{l}(s) \underset{k \rightarrow 0}{\sim} a_{l}^{(I)}+b_{l}^{(I)} k^{2}+\cdots, \\
k=\sqrt{s / 4-M_{\pi}^{2}} .
\end{gathered}
$$

\section{THE $\pi \pi$ SCATTERING AMPLITUDES AT LOW ENERGY $\left(s^{1 / 2} \leq 1.42 \mathrm{GeV}\right)$}

We present in this section parametrizations of the $\mathrm{S}, \mathrm{P}$, $\mathrm{D}, \mathrm{F}$, and $\mathrm{G}$ waves in $\pi \pi$ scattering obtained fitting experimental data at low energy, $s^{1 / 2} \leq 1.42 \mathrm{GeV}$, which will provide a representation of the experimental $\pi \pi$ scattering amplitudes in this energy range: this may be considered as an energy-dependent phase shift analysis. Above $1.42 \mathrm{GeV}$, we will use the Regge expressions obtained in Ref. [5], which we reproduce and discuss in Appendix B in the present paper. In following sections we will verify to which extent the scattering amplitudes that one finds in this way are consistent with dispersion relations or crossing symmetry, within the errors given.

Before entering into the actual fits, a few words are due on the choice of the energy $1.42 \mathrm{GeV}$ as limit between the regions where we use phase shift analyses and Regge representations. Experimental phase shift analyses exist up to $\sim 2 \mathrm{GeV}$. However, as is known, phase shift analyses become ambiguous as soon as inelastic processes become important. As we will discuss in some detail in Appendix C, the existing experimental phase shift analyses become suspect for energies above $\sim 1.4 \mathrm{GeV}$ : in particular, they produce cross sections that deviate from experimentally measured total cross sections.

As for the higher energy range, we have shown in Ref. [5] that a Regge description of the imaginary part of the $\pi \pi$ scattering amplitudes agrees well with $\pi \pi$ cross section data (and, through factorization, also with $\pi N$ and $N N$ data) for kinetic energies above $\sim 1 \mathrm{GeV}$; for $\pi \pi$ scattering, down to $s^{1 / 2} \simeq 1.4$, or even to $1.3 \mathrm{GeV}$, depending on the process. We have chosen the limiting energy to be $1.42 \mathrm{GeV}$ as a reasonable compromise; but one could have taken lower limits, or slightly higher ones, as well. In fact, it is not possible to go below $1.4 \mathrm{GeV}$ with a Regge description for the $I_{t}=1$ amplitude or for $\pi^{0} \pi^{0}$ scattering, since both contain the isospin zero amplitudes, which show two resonances just below that region [the $f_{2}(1270)$ and the $\left.f_{0}(1370)\right]$. But it is possible to choose a lower junction point between the phase shift analyses and the Regge formulas for $\pi^{0} \pi^{+}$scattering. The influence of this is negligible for $\pi^{0} \pi^{+}$, as we will show in Sec. III A 2, provided one stays in the range $1.32 \mathrm{GeV} \leq s^{1 / 2} \leq$ $1.42 \mathrm{GeV}$, with the larger values favored.

We now turn to the parametrizations. In writing them we will use the requirements of analyticity and elastic unitarity. Extra information has been added to help stabilize the fits in those channels where the low energy data are scarce; in particular, information on Adler zeros or scattering lengths. For the latter we impose values obtained from their Froissart-Gribov representation, but with enlarged error bars.

The method used to take into account unitarity and analyticity will be the standard one of the effective range formalism, supplemented by a conformal expansion. To be precise, for a given partial wave $f_{l}^{(I)}(s)$ we write, for any value (complex or real) of $s$,

$$
\begin{aligned}
f_{l}^{(I)}(s) & =\frac{2 s^{1 / 2}}{\pi k} \frac{1}{2 s^{1 / 2} k^{-2 l-1} \Phi_{l}^{(I)}(s)-i} \\
& =\frac{k^{2 l}}{\pi} \frac{1}{\Phi_{l}^{(I)}(s)-i k^{2 l+1} / 2 s^{1 / 2}} .
\end{aligned}
$$

The effective range function $\Phi_{l}^{(I)}(s)$ is real on the segment $0 \leq s \leq s_{0}$, but it will be complex above $s_{0}$, and also for $s \leq 0$. Here $s_{0}$ is the energy squared above which inelastic processes are nonnegligible. Using only the requirements of causality and conservation of probability, it can be shown that $\Phi_{l}^{(I)}(s)$ is analytic in the complex $s$ plane, cut from $-\infty$ to 0 and from $s_{0}$ to $+\infty$; see Fig. 1 .

We can profit from these analytical properties as follows. We map the cut plane into the unit disk (as in Fig. 1) by means of the conformal transformation

$$
w(s)=\frac{\sqrt{s}-\sqrt{s_{0}-s}}{\sqrt{s}+\sqrt{s_{0}-s}} .
$$

The properties of analyticity and elastic unitarity of $f_{l}^{(I)}(s)$ are then strictly equivalent to uniform convergence (in the variable $w$ ) in the disk $|w|<1$ of the series

$$
\Phi_{l}^{(I)}(s)=\sum_{n=0}^{\infty} B_{n} w(s)^{n} .
$$

This series thus converges, in the variable $s$, uniformly in the interior of the whole cut plane; in general very quickly. It will turn out that we will need only two or three terms in the expansions for the partial wave amplitudes.
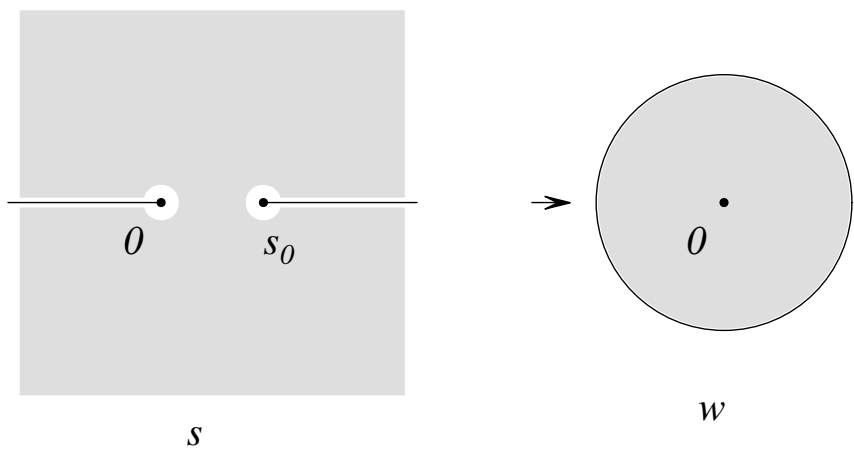

W

FIG. 1. The mapping $s \rightarrow w$. 
Before starting with the actual fits, we here say a few words on the values of $s_{0}$ (i.e., the energy at which we consider inelasticity not negligible) that we will take for the various waves. For the $\mathrm{S} 0$ wave, the $\bar{K} K$ channel is strongly coupled, so here $s_{0}=4 m_{K}^{2}$. For other waves the $\bar{K} K$ channel is weakly coupled. In the cases where we have sufficiently precise data (that is, for the $\mathrm{P}$ wave and the low energy $\mathrm{S} 2$ wave), we will take $s_{0}^{1 / 2}=1.05 \mathrm{GeV}$ which is, approximately, the $2 \pi \rho$ threshold.

For the D0 wave, and for the S2 wave at intermediate energy, $1.05 \mathrm{GeV} \lesssim s^{1 / 2} \lesssim 1.4 \mathrm{GeV}$, inelasticity is detected. It is small (but not negligible) while, unfortunately, the data are not precise enough to perform a fully consistent analysis. We then follow a different strategy: we fit the experimental phase shifts using formulas that neglect inelasticity below $1.45 \mathrm{GeV}$ (which is, approximately, the $2 \rho$ threshold). The experimental inelasticity between 1 and $1.42 \mathrm{GeV}$ is then added by hand.

Finally, for the D2, F waves, for which experiments detect no inelasticity below $1.42 \mathrm{GeV}, s_{0}$ is taken equal to $1.45 \mathrm{GeV}$.

\section{A. The $P$ wave}

\section{Parametrization of the $P$ wave below $1 \mathrm{GeV}$}

We will consider first the $\mathrm{P}$ wave for $\pi \pi$ scattering below $1 \mathrm{GeV}$, to exemplify the methods, because it is the one for which more precise results are obtained. We start thus considering the region of energies where the inelasticity is below the $2 \%$ level; say, $s_{0}^{1 / 2} \leq 1.05 \mathrm{GeV}^{2}$.

We then expand $\Phi_{1}(s)$ in powers of $w$, and, reexpressing $w$ in terms of $s$, the expansion will be convergent over all the cut $s$ plane. Actually, and because we know that the $\mathrm{P}$ wave resonates at $s=M_{\rho}^{2}$, it is more convenient to expand not $\Phi_{1}(s)$ itself, but $\psi(s)$ given by

$$
\Phi_{1}(s)=\left(s-M_{\rho}^{2}\right) \psi(s) / 4
$$

so we write

$$
\psi(s)=\left\{B_{0}+B_{1} w+\cdots\right\} .
$$

In terms of $\Phi_{1}(s)$ we thus find the expression for the cotangent of the phase shift, keeping two terms in the expansion,

$$
\begin{aligned}
\cot \delta_{1}(s) & =\frac{s^{1 / 2}}{2 k^{3}}\left(M_{\rho}^{2}-s\right)\left\{B_{0}+B_{1} \frac{\sqrt{s}-\sqrt{s_{0}-s}}{\sqrt{s}+\sqrt{s_{0}-s}}\right\} ; \\
s_{0}^{1 / 2} & =1.05 \mathrm{GeV} .
\end{aligned}
$$

$M_{\rho}, B_{0}, B_{1}$ are free parameters to be fitted to experiment. In terms of $\Phi_{1}, \psi$ we have, for the rho width, and the scattering length, $a_{1}$,

$$
\begin{aligned}
& \Gamma_{\rho}=\frac{2 k_{\rho}^{3}}{M_{\rho}^{2} \psi\left(M_{\rho}^{2}\right)}, \quad k_{\rho}=\frac{1}{2} \sqrt{M_{\rho}^{2}-4 M_{\pi}^{2}}, \\
& a_{1}=\frac{-1}{4 M_{\pi} \Phi_{1}\left(4 M_{\pi}^{2}\right)}=\frac{1}{M_{\pi}\left(M_{\rho}^{2}-4 M_{\pi}^{2}\right) \psi\left(4 M_{\pi}^{2}\right)} .
\end{aligned}
$$

The values $B_{0}=$ const, $B_{i \geq 1}=0$ would correspond to a perfect Breit-Wigner. Actually, it is known that the $\rho$ deviates from a pure Breit-Wigner and for a precision parametrization two terms, $B_{0}$ and $B_{1}$, have to be kept in (2.3). Note that the parametrization holds not only on the physical region $4 M_{\pi}^{2} \leq s \leq s_{0}$, but on the unphysical region $0 \leq s \leq 4 M_{\pi}^{2}$ and also over the whole region of the complex $s$ plane with $s \neq 0$. The parametrization given now is the one that has less biases, in the sense that no model has been used: we have imposed only the highly safe requirements of analyticity and unitarity, which follow from causality and conservation of probability.

The best values for our parameters are actually obtained not from fits to $\pi \pi$ scattering data, but from fits to the pion form factor [9]. This is obtained from $e \pi$ scattering, from $e^{+} e^{-} \rightarrow \pi^{+} \pi^{-}$and from $\tau \rightarrow \nu \pi \pi$ decay, where we have a large number of precise data, and pions are on their mass shells. Including systematic experimental errors in the fits, and including in the fit also the constraint $a_{1}=(38 \pm 3) \times$ $10^{-3} M_{\pi}^{-3}$ for the scattering length, we have

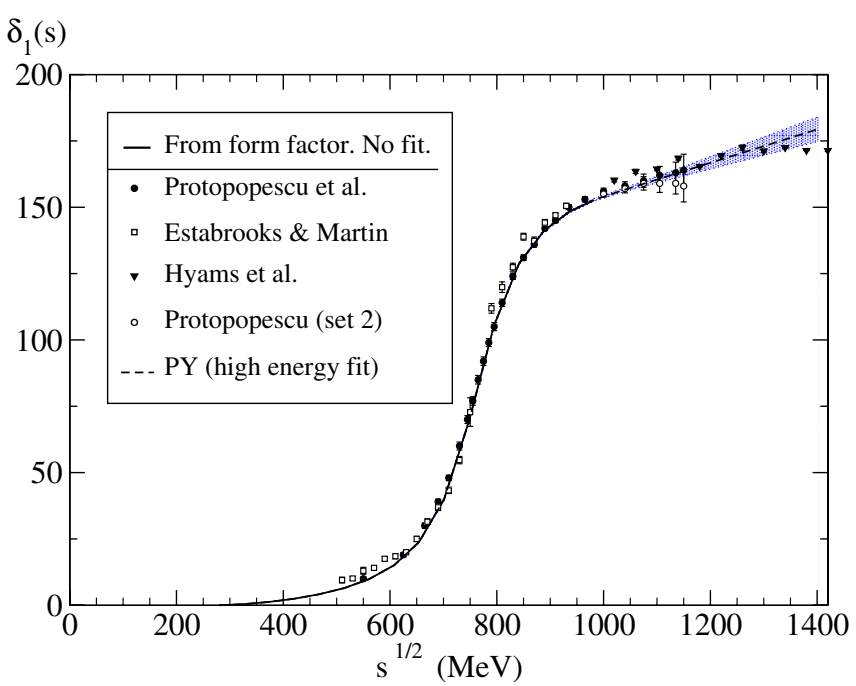

FIG. 2 (color online). The phase shifts of solution 1 from Protopopescu et al. [10], Hyams et al. [11(a)], and Estabrooks and Martin [11(a)] compared with the prediction with the parameters (2.6) (solid line below $1 \mathrm{GeV}$ ). We emphasize that this is not a fit to these experimental data, but is obtained from the pion form factor [9]. The error here is like the thickness of the line. Above $1 \mathrm{GeV}$, the dotted line and error (PY) are as follows from the fit (2.7). 


$$
\begin{gathered}
\text { from } \pi^{+} \pi^{-}: B_{0}=1.074 \pm 0.006, \quad B_{1}=0.13 \pm 0.04 ; \quad M_{\rho}=774.0 \pm 0.4 \mathrm{MeV} \\
\text { from } \pi^{+} \pi^{0}: B_{0}=1.064 \pm 0.006, \quad B_{1}=0.13 \pm 0.04 ; \quad M_{\rho}=773.1 \pm 0.6 \mathrm{MeV}
\end{gathered}
$$

The fit is excellent; one has $\chi^{2} /$ d.o.f. $=245 /(244-13)$, and the slight excess over unity is due to the well-known incompatibility of OPAL and other data for $\tau$ decay at low invariant mass.

The corresponding values for the rho width, and $\mathrm{P}$ wave scattering length and effective range parameter, are

$$
\begin{aligned}
& a_{1}=(37.8 \pm 0.8) \times 10^{-3} M_{\pi}^{-3}, \quad b_{1}=(4.74 \pm 0.09) \times 10^{-3} M_{\pi}^{-5}, \quad \Gamma_{\rho^{0}}=146.0 \pm 0.8 ; \quad \text { from } \pi^{+} \pi^{-} \\
& a_{1}=(37.8 \pm 0.8) \times 10^{-3} M_{\pi}^{-3}, \quad b_{1}=(4.78 \pm 0.09) \times 10^{-3} M_{\pi}^{-5}, \quad \Gamma_{\rho^{+}}=147.7 \pm 0.7 ; \quad \text { from } \pi^{+} \pi^{0} .
\end{aligned}
$$

Although the values of the experimental $\pi \pi$ phase shifts were not included in the fit, the phase shifts that (2.5a) implies are in very good agreement with them, as shown in Fig. 2.

If neglecting violations of isospin invariance, one would expect numbers equal to the average of both sets of parameters in (2.5a). If we also increase the error, so as to take into account that due to isospin breaking, by adding linearly half the difference between both determinations (2.5a), we find

$$
\begin{gathered}
B_{0}=1.069 \pm 0.011, \quad B_{1}=0.13 \pm 0.05, \\
M_{\rho}=773.6 \pm 0.9, \quad a_{1}=(37.6 \pm 1.1) \times 10^{-3} M_{\pi}^{-3}, \\
b_{1}=(4.73 \pm 0.26) \times 10^{-3} M_{\pi}^{-5} ;
\end{gathered}
$$

the corresponding parametrization we take to be valid up to $s^{1 / 2}=1 \mathrm{GeV}$.

\section{The $P$ wave for $1 \mathrm{GeV} \leq s^{1 / 2} \leq 1.42 \mathrm{GeV}$}

In the range $1 \mathrm{GeV} \leq s^{1 / 2} \leq 1.42 \mathrm{GeV}$ one is sufficiently far away from thresholds to neglect their influence (the coupling to $\bar{K} K$ is negligible). A purely empirical parametrization that agrees with the data in Protopopescu et al. [10] and Hyams et al. [11(a)] up to $1.42 \mathrm{GeV}$, within errors, is obtained with a linear fit to the phase and inelasticity:

$$
\begin{aligned}
& \delta_{1}(s)=\lambda_{0}+\lambda_{1}(\sqrt{s / \hat{s}}-1), \quad \eta_{1}(s)=1-\epsilon(\sqrt{s / \hat{s}}-1) ; \\
& \epsilon=0.30 \pm 0.15, \quad \lambda_{0}=2.69 \pm 0.01, \quad \lambda_{1}=1.1 \pm 0.2 .
\end{aligned}
$$

Here $\hat{s}=1 \mathrm{GeV}^{2}$. The value of $\lambda_{0}$ ensures the agreement of the phase with the value given in the previous subsection at $s=\hat{s} \equiv 1 \mathrm{GeV}^{2}$. This fit is good (Fig. 2).

It should be remarked, however, that there are other solutions for the $\mathrm{P}$ wave above $1.15 \mathrm{GeV}$. In the first analysis of Hyams et al. [11(a)], that we have followed here, a resonance occurs with a mass $\sim 1.6 \mathrm{GeV}$, and its effect is only felt above $\sim 1.4 \mathrm{GeV}$; but in the second analysis by the same group, Hyams et al. [11(b)] a broad, highly inelastic resonance (actually, more a spike than a resonance) with a mass $\sim 1.35 \mathrm{GeV}$ appears, or does not appear, depending on the solution chosen. Finally, the Particle Data Group (based mostly on evidence from $e^{+} e^{-}$annihilation and $\tau$ decay) report a resonance at $1.45 \mathrm{GeV}$ : one has to admit that the situation for the $\mathrm{P}$ wave above $1.15 \mathrm{GeV}$ is not clear. We will return to this later.

\section{B. The $S$ waves}

\section{Parametrization of the $S$ wave for $I=2$}

We consider three sets of experimental data. The first corresponds to solution A in the paper by Hoogland et al. [12], who use the reaction $\pi^{+} p \rightarrow \pi^{+} \pi^{+} n$. We will not consider the so-called solution $\mathrm{B}$ in this paper; while it produces results similar to the other, its errors are clearly underestimated. The second set corresponds to the work of Losty et al., [12] who analyze instead $\pi^{-} p \rightarrow \pi^{-} \pi^{-} \Delta$.

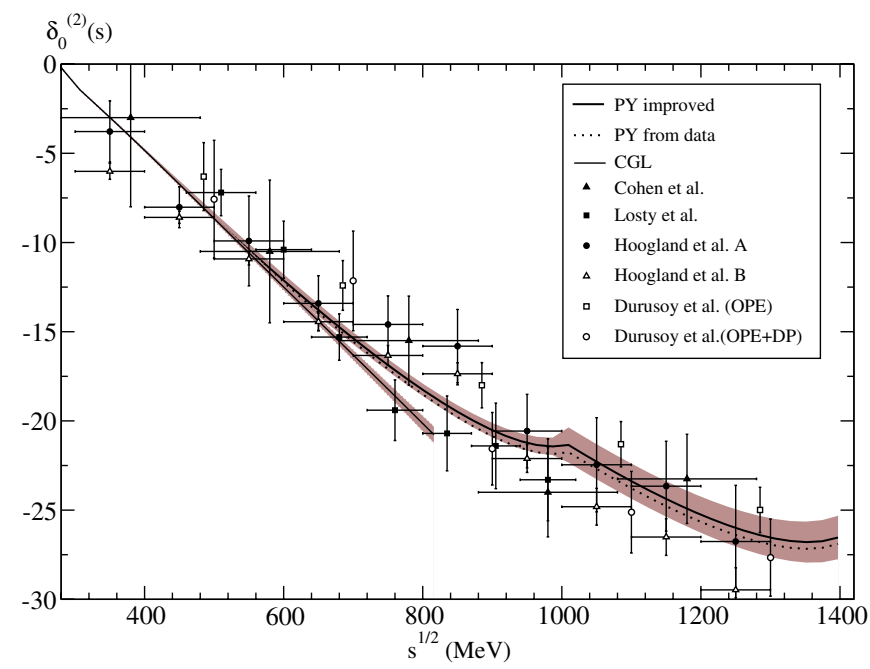

FIG. 3 (color online). Continuous line: The $I=2, S$-wave phase shifts, and error bands, corresponding to (2.7), (2.8a), (2.8b), and (2.9), denoted by PY. Dotted line: the fit from Ref. [6]. The experimental points are from Losty et al. (black squares), Hoogland et al. [12] solution A (black dots), and Cohen et al. [4] (black triangles). We have also included data from Durusoy et al. and from solution B of Hoogland et al., although they were not used in the fits. The dashed line, which lies below our fit, is the S2 phase of Colangelo, Gasser, and Leutwyler [2] with error band attached. 
The third set are the data of Cohen et al. [4], who consider $\pi^{-} n \rightarrow \pi^{-} \pi^{-} p$. These three sets represent a substantial improvement over other determinations; since they produce two like charge pions, only isospin 2 contributes, and one gets rid of the large S0 wave contamination. However, they still present the problem that one does not have scattering of real pions.

For isospin 2, there is no low energy resonance, but $f_{0}^{(2)}(s)$ presents the feature that a zero is expected (and, indeed, confirmed by the fits) in the region $0<s<4 M_{\pi}^{2}$. This zero of $f_{0}^{(2)}(s)$ is related to the so-called Adler zeros and, to lowest order in chiral perturbation theory, occurs at $s=2 z_{2}^{2}$ with $z_{2}=M_{\pi}$. We note that, unlike the corresponding zero for the S0 wave, $2 z_{2}^{2}$ is inside the region where the conformal expansion is expected to converge.

In Ref. [6], we neglected inelasticity below 1.45 , and fitted all experimental data, for $s^{1 / 2} \leq 1380 \mathrm{MeV}$. A more precise determination (and, above all, with more realistic error estimates) is obtained if we realize that inelasticity is detected experimentally above the $2 \pi \rho$ threshold, $\hat{s}^{1 / 2}=$ $1.05 \mathrm{GeV}$, so we should fit separately the low and high energy regions (Fig. 3).

In the low energy region, we fix $z_{2}=M_{\pi}$ and fit only the low energy data, $s^{1 / 2}<1.0 \mathrm{GeV}$; later, in Sec. IV, we will allow $z_{2}$ to vary. We write

$$
\begin{gathered}
\cot \delta_{0}^{(2)}(s)=\frac{s^{1 / 2}}{2 k} \frac{M_{\pi}^{2}}{s-2 z_{2}^{2}}\left\{B_{0}+B_{1} \frac{\sqrt{s}-\sqrt{\hat{s}-s}}{\sqrt{s}+\sqrt{\hat{s}-s}}\right\}, \\
z_{2} \equiv M_{\pi} ; \quad \hat{s}^{1 / 2}=1.05 \mathrm{GeV}
\end{gathered}
$$

Then we get $\chi^{2} /$ d.o.f. $=13.0 /(25-2)$ and

$$
\begin{gathered}
B_{0}=-80.4 \pm 2.8, \quad B_{1}=-73.6 \pm 12.6 \\
a_{0}^{(2)}=(-0.052 \pm 0.012) M_{\pi}^{-1} \\
b_{0}^{(2)}=(-0.085 \pm 0.011) M_{\pi}^{-3}
\end{gathered}
$$

For the high energy region we neglect the inelasticity below $1.45 \mathrm{GeV}$, and then add inelasticity by hand. We consider two extreme possibilities: fitting the whole range, or fitting only high energy data $\left(s^{1 / 2} \geq 0.91 \mathrm{GeV}\right)$, requiring agreement of the central value with the low energy determination at $s^{1 / 2}=1 \mathrm{GeV}$. We accept as the best result an average of the two, and thus have

$$
\begin{gathered}
\cot \delta_{0}^{(2)}(s)=\frac{s^{1 / 2}}{2 k} \frac{M_{\pi}^{2}}{s-2 M_{\pi}^{2}}\left\{B_{0}+B_{1} \frac{\sqrt{s}-\sqrt{s_{0}-s}}{\sqrt{s}+\sqrt{s_{0}-s}}\right\} \\
s_{0}^{1 / 2}=1.45 \mathrm{GeV} ; \quad B_{0}=-123 \pm 6, \quad B_{1}=-118 \pm 14,
\end{gathered}
$$

and we have enlarged the errors to cover both extreme cases. We will not consider varying the position of the Adler zero for this high energy piece.

The inelasticity may be described by the empirical fit

$$
\eta_{0}^{(2)}(s)= \begin{cases}1-\epsilon(1-\hat{s} / s)^{3 / 2}, & \epsilon=0.18 \pm 0.12 ; s>\hat{s}=(1.05 \mathrm{GeV})^{2} \\ 1, & s<\hat{s}=(1.05 \mathrm{GeV})^{2}\end{cases}
$$

These formulas are expected to hold from $s^{1 / 2}=1.0 \mathrm{GeV}$ up to $1.42 \mathrm{GeV}$. As shown in Fig. 3, both determinations, (2.8a), (2.8b), and (2.9) and that in Ref. [6] are almost overlapping (their error bands actually overlap).

\section{Parametrization of the $S$ wave for $I=0$ below $0.95 \mathrm{GeV}$ (global fit)}

The $\mathrm{S}$ wave with isospin zero is difficult to deal with. Here we have a very broad enhancement, variously denoted as $\epsilon, \sigma, f_{0}$, around $s^{1 / 2}=\mu_{0} \sim 800 \mathrm{MeV}$. We will not discuss here whether this enhancement is a bona fide resonance; we merely remark that in all experimental phase shift analyses $\delta_{0}^{(0)}(s)$ crosses $90^{\circ}$ somewhere between 600 and $900 \mathrm{MeV}$; we define $\mu_{0}$ as the energy at which the phase equals $90^{\circ}$. Moreover, we expect a zero of $f_{0}^{(0)}(s)$ (Adler zero), hence a pole of the effective range function $\Phi_{0}^{(0)}(s)$, for $s=\frac{1}{2} z_{0}^{2}$ with $\frac{1}{2} z_{0}^{2}$ in the region $0<s<4 M_{\pi}^{2}$. Chiral perturbation theory suggests that $z_{0} \simeq M_{\pi}$.
We can distinguish two energy regions: below $s_{0}^{1 / 2}=$ $2 m_{K}$ we are under the $\bar{K} K$ threshold. Between $s_{0}^{1 / 2}$ and $s^{1 / 2} \sim 1.2$ there is a non-negligible coupling between the $\bar{K} K$ and $\pi \pi$ channels and the analysis becomes very unstable, because there is little information on the process $\pi \pi \rightarrow \bar{K} K$ and even less on $\bar{K} K \rightarrow \bar{K} K$. We will present later an empirical fit in the region of energies around and above $1 \mathrm{GeV}$, and we will now concentrate in the low energy region.

For the theoretical formulas we impose the Adler zero at $s=\frac{1}{2} M_{\pi}^{2}$ (no attempt is made to vary this for the moment; see Sec. IV), and a zero of $\cot \delta_{0}^{(0)}(s)$ at $s=\mu_{0}^{2}, \mu_{0}$ a free parameter. Then we map the $s$ plane, cut along the left hand cut $(s \leq 0)$ and the $\bar{K} K$ cut, writing

$$
\cot \delta_{0}^{(0)}(s)=\frac{s^{1 / 2}}{2 k} \frac{M_{\pi}^{2}}{s-\frac{1}{2} z_{0}^{2}} \frac{\mu_{0}^{2}-s}{\mu_{0}^{2}}\left\{B_{0}+B_{1} w(s)+\cdots\right\},
$$

and 


$$
w(s)=\frac{\sqrt{s}-\sqrt{s_{0}-s}}{\sqrt{s}+\sqrt{s_{0}-s}}, \quad s_{0}=4 m_{K}^{2}
$$

(we have taken $m_{K}=0.496 \mathrm{GeV}$ ).

This parametrization does not represent fully the coupling of the $\bar{K} K$ channel and we will thus only take it to be valid up to $s^{1 / 2}=0.95 \mathrm{GeV}$.

On the experimental side the situation is still a bit confused, although it has cleared up substantially in recent years thanks to the experimental information on $K_{l 4}$ and $K_{2 \pi}$ decays. The information we have on this S0 wave is of three kinds: from phase shift analysis in collisions [10,11(a)] $\pi p \rightarrow \pi \pi N, \Delta$; from the decay [13] $K_{l 4}$; and from $K_{2 \pi}$ decays. The last gives the value of the combination $\delta_{0}^{(0)}-\delta_{0}^{(2)}$ at $s^{1 / 2}=m_{K}$; the decay $K_{l 4}$ gives $\delta_{0}^{(0)}-\delta_{1}$ at low energies, $s^{1 / 2} \lesssim 380 \mathrm{MeV}$. If using recent $K_{2 \pi}$ information [14] combined with older determinations, and with the $I=2$ phase obtained in the previous subsection, one finds the phase

$$
\delta_{0}^{(0)}\left(m_{K}^{2}\right)=43.3 \pm 3^{\circ}
$$

We will here include in the fit the low energy data from $K_{l 4}$ decay $^{1}$ shown in Fig. 4, and we impose the value of $\delta_{0}^{(0)}\left(m_{K}^{2}\right)$ from $K_{2 \pi}$ given in (2.11). The main virtue of these $K$ decay data is that they refer to pions on their mass shell; but, unfortunately, this leaves too much room at the upper energy range, $s^{1 / 2} \gtrsim 0.6 \mathrm{GeV}$. If we fit only $K$ decay data we can only use one-parameter $B_{0}$ in the conformal expansion: if including another parameter, spurious minima would appear. We get a good fit, albeit with rather large errors:

$$
\begin{gathered}
\cot \delta_{0}^{(0)}(s)=\frac{s^{1 / 2}}{2 k} \frac{M_{\pi}^{2}}{s-\frac{1}{2} z_{0}^{2}} \frac{\mu_{0}^{2}-s}{\mu_{0}^{2}} B_{0}, \quad z_{0} \equiv M_{\pi} ; \\
B_{0}=18.5 \pm 1.7, \quad \mu_{0}=766 \pm 95 \mathrm{MeV} \\
\frac{\chi^{2}}{\text { d.o.f. }}=\frac{5.7}{12-2} . \quad a_{0}^{(0)}=(0.22 \pm 0.02) \times M_{\pi}^{-1} ; \\
\delta_{0}^{(0)}\left(m_{K}\right)=43 \pm 5^{\circ} .
\end{gathered}
$$

To improve on this we have to add further data (and one more parameter $B_{1}$ ). To do so, we can follow two different procedures. We can add to the $K_{e 4}, K_{2 \pi}$ data various sets of experimental phase shifts, fitting each set individually; this we will do in Sec. III. Or we can follow what we consider to be the best procedure: we combine in the fit data from

\footnotetext{
${ }^{1}$ As a technical point, we mention that we have increased by $50 \%$ the error in the point at highest energy, $s^{1 / 2}=381.4 \mathrm{MeV}$, from the $K_{e 4}$ compilation of Pislak et al., because this experimental value represents an average over a long energy range that extends to the edge of phase space.
}

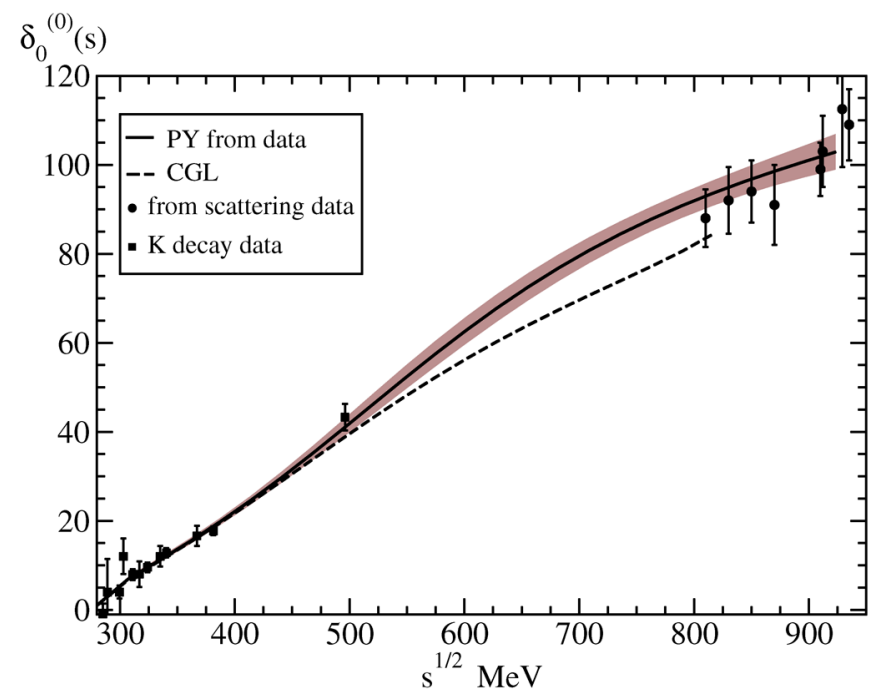

FIG. 4 (color online). The $I=0, S$-wave phase shifts and error bands corresponding to Eq. (2.14a) and (2.14b) (PY, continuous line). Also shown (black dots) are the points from $K_{l 4}$ and $K_{2 \pi}$ decays, and only the high energy data included in the fits, as given in Eqs. (2.13a)-(2.13c). The dashed line is the solution of Colangelo et al. [2]

various experiments at energies above $0.8 \mathrm{GeV}$, which we will call a global fit. The reason to choose only data above $0.8 \mathrm{GeV}$ is that, in the region between $0.81 \mathrm{GeV}$ and $0.97 \mathrm{GeV}$, the more relevant experimental results have overlapping error bars, something that does not happen at other energies (see Fig. 5). By combining several sets we may expect to average out systematic errors, at least to some extent.

At high energy we thus include the following sets of data: first of all, the values

$$
\begin{aligned}
& \delta_{0}^{(0)}\left(0.870^{2} \mathrm{GeV}^{2}\right)=91 \pm 9^{\circ} \\
& \delta_{0}^{(0)}\left(0.910^{2} \mathrm{GeV}^{2}\right)=99 \pm 6^{\circ} \\
& \delta_{0}^{(0)}\left(0.935^{2} \mathrm{GeV}^{2}\right)=109 \pm 8^{\circ} \\
& \delta_{0}^{(0)}\left(0.965^{2} \mathrm{GeV}^{2}\right)=134 \pm 14^{\circ}
\end{aligned}
$$

These points are taken from solution 1 of Protopopescu et al. [10] (both with and without modified moments), with the error increased by the difference between this and solution 3 data in the same reference. We will also include in the fit the data, at similar energies, of Grayer et al. [11(a)]

$$
\begin{aligned}
& \delta_{0}^{(0)}\left(0.912^{2} \mathrm{GeV}^{2}\right)=103 \pm 8^{\circ} \\
& \delta_{0}^{(0)}\left(0.929^{2} \mathrm{GeV}^{\circ}\right)=112.5 \pm 13^{\circ} \\
& \delta_{0}^{(0)}\left(0.952^{2} \mathrm{GeV}^{2}\right)=126 \pm 16^{\circ} \\
& \delta_{0}^{(0)}\left(0.970^{2} \mathrm{GeV}^{2}\right)=141 \pm 18^{\circ}
\end{aligned}
$$

The central values are obtained averaging the solutions 


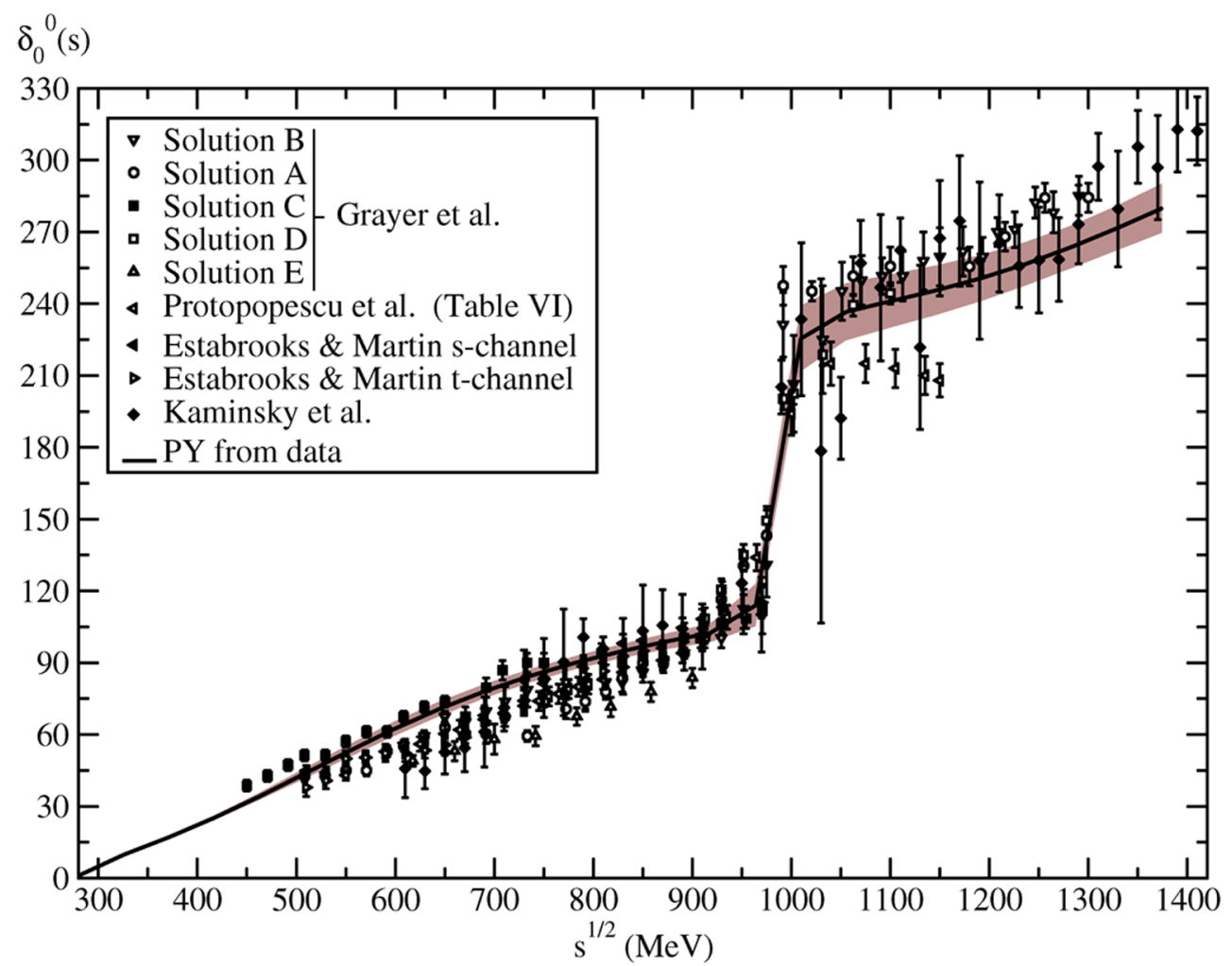

FIG. 5 (color online). The $I=0, S$-wave phase shifts and error band in the whole energy range as given by our fits (PY), Eqs. (2.14a), (2.14b), (2.15a), (2.15b), and (2.15b'). The experimental points from $K_{l 4}$ and $K_{2 \pi}$ decays are not shown.

given by Grayer et al., except ${ }^{2}$ solution E, and the error is calculated adding quadratically the statistical error of the highest point, the statistical error of the lowest point (for each energy) and the difference between the central value and the farthest point.

Finally, we add three points between 0.8 and $0.9 \mathrm{GeV}$ obtained averaging the $s$-channel solution of Estabrooks and Martin and solution 1 of Protopopescu et al., which represent two extremes. The error is obtained adding the difference between these two in quadrature to the largest statistical error. In this way we obtain the numbers,

$$
\begin{aligned}
& \delta_{0}^{(0)}\left(0.810^{2} \mathrm{GeV}^{2}\right)=88 \pm 6^{\circ} ; \\
& \delta_{0}^{(0)}\left(0.830^{2} \mathrm{GeV}^{2}\right)=92 \pm 7^{\circ} \\
& \delta_{0}^{(0)}\left(0.850^{2} \mathrm{GeV}^{2}\right)=94 \pm 6^{\circ} .
\end{aligned}
$$

In spite of the generous errors taken, it should be noted that these data could still contain systematic errors, beyond those taken into account in (2.13a)-(2.13c), which may contaminate the results of the fit at the higher energies. In Sec. IV we will use dispersion relations to improve the parametrization of this S0 wave.

\footnotetext{
${ }^{2}$ Solution $\mathrm{E}$ is incompatible with all other data sets in the region above $\sim 0.8 \mathrm{GeV}$; we will discuss this in more detail below.
}

We now have enough constraints to include two parameters $B_{i}$ in the expansion of $\cot \delta_{0}^{(0)}$. We find

$$
\begin{gathered}
\cot \delta_{0}^{(0)}(s)=\frac{s^{1 / 2}}{2 k} \frac{M_{\pi}^{2}}{s-\frac{1}{2} z_{0}^{2}} \frac{\mu_{0}^{2}-s}{\mu_{0}^{2}}\left\{B_{0}+B_{1} \frac{\sqrt{s}-\sqrt{s_{0}-s}}{\sqrt{s}+\sqrt{s_{0}-s}}\right\} \\
z_{0} \equiv M_{\pi} ; \quad B_{0}=21.04, \quad B_{1}=6.62 \\
\mu_{0}=782 \pm 24 \mathrm{MeV} ; \quad \chi^{2} / \text { d.o.f. }=15.7 /(19-3) . \\
a_{0}^{(0)}=(0.230 \pm 0.010) M_{\pi}^{-1}, \\
b_{0}^{(0)}=(0.268 \pm 0.011) M_{\pi}^{-3} ; \\
\delta_{0}^{(0)}\left(m_{K}\right)=41.0^{\circ} \pm 2.1^{\circ} ;
\end{gathered}
$$

this fit (shown in Fig. 4) we take to be valid for $s^{1 / 2} \leq$ $0.95 \mathrm{GeV}$. The errors of the $B_{i}$ are strongly correlated; uncorrelated errors are obtained if replacing the $B_{i}$ by the parameters $x, y$ with

$$
\begin{aligned}
& B_{0}=y-x ; \quad B_{1}=6.62-2.59 x ; \\
& y=21.04 \pm 0.70, \quad x=0 \pm 2.6 .
\end{aligned}
$$

\section{Parametrization of the $S$ wave for $I=0$ below $0.95 \mathrm{GeV}$ (individual fits)}

In this subsection we summarize, in Table I, the results of fits to data from $K_{l 4}$ and $K_{2 \pi}$ decays including also, individually, data from various sets of phase shift analyses. 
TABLE I. PY, Eqs. (2.14a) and (2.14b): our global fit, Sec. II B 2. The rest are fits to either $K$ decay data alone, or combining these with various $\pi \pi$ scattering data sets. Grayer A, B, C, E: the solutions in the paper of Grayer et al. [11(a)] (solution D only concerns data above $1 \mathrm{GeV}$ ). EM: the solutions of Estabrooks and Martin [11(a)]. Kaminski: the papers of Kamiński et al. [11(c)] Protopopescu VI, XII, and VIII: the corresponding solutions in Ref. [10]. Solutions A, B and C of Grayer et al., as well as both solutions EM and Kamiński et al. are from energy-independent analyses. The rest are from energy-dependent evaluations. (a) We do not give errors here as they are strongly correlated; cf. Eq. (2.14b).

\begin{tabular}{|c|c|c|c|c|}
\hline & $B_{0}$ & $B_{1}$ & $\mu_{0}(\mathrm{MeV})$ & $M_{\pi} \times a_{0}^{(0)}$ \\
\hline PY, Eqs. (2.14a) and (2.14b) & $21.04^{\mathrm{a}}$ & $6.62^{\mathrm{a}}$ & $782 \pm 24$ & $0.230 \pm 0.010$ \\
\hline$K$ decay only & $18.5 \pm 1.7$ & $\equiv 0$ & $766 \pm 95$ & $0.218 \pm 0.021$ \\
\hline$K$ decay data + Grayer, $\mathrm{B}$ & $22.7 \pm 1.6$ & $12.3 \pm 3.7$ & $858 \pm 15$ & $0.246 \pm 0.042$ \\
\hline$K$ decay data + Grayer, $\mathrm{C}$ & $16.8 \pm 0.85$ & $-0.34 \pm 2.34$ & $787 \pm 9$ & $0.236 \pm 0.023$ \\
\hline$K$ decay data + Grayer, $\mathrm{E}$ & $21.5 \pm 3.6$ & $12.5 \pm 7.6$ & $1084 \pm 110$ & $0.26 \pm 0.05$ \\
\hline$K$ decay data + Kaminski & $27.5 \pm 3.0$ & $21.5 \pm 7.4$ & $789 \pm 18$ & $0.25 \pm 0.10$ \\
\hline$K$ decay data + Grayer, $\mathrm{A}$ & $28.1 \pm 1.1$ & $26.4 \pm 2.8$ & $866 \pm 6$ & $0.29 \pm 0.04$ \\
\hline$K$ decay data + EM, $s$ channel & $29.8 \pm 1.3$ & $25.1 \pm 3.3$ & $811 \pm 7$ & $0.27 \pm 0.05$ \\
\hline$K$ decay data $+\mathrm{EM}, t$ channel & $29.3 \pm 1.4$ & $26.9 \pm 3.4$ & $829 \pm 6$ & $0.27 \pm 0.05$ \\
\hline$K$ decay data + Protopopsecu VI & $27.0 \pm 1.7$ & $22.0 \pm 4.1$ & $855 \pm 10$ & $0.26 \pm 0.05$ \\
\hline$K$ decay data + Protopopsecu XII & $25.5 \pm 1.7$ & $18.5 \pm 4.1$ & $866 \pm 14$ & $0.25 \pm 0.05$ \\
\hline$K$ decay data + Protopopsecu VIII & $27.1 \pm 2.3$ & $23.8 \pm 5.0$ & $913 \pm 18$ & $0.27 \pm 0.07$ \\
\hline
\end{tabular}

${ }^{\mathrm{a}}$ We do not give errors here as they are strongly correlated; cf. Eq. (2.14b).

The method of fit is like that used in the previous subsection; in particular, we have fixed the Adler zero at $z_{0}=$ $M_{\pi}$ in all these fits.

A few comments are in order. First of all, we note that the solution E of Grayer et al., as well as what one finds with only $K$ decay data, have very large errors. Moreover, solution $E$ of Grayer et al. only contains eight points which clearly lie below all other experimental data; see Fig. 5. Second, it is seen that the values of the parameters in the first five fits (which, as will be shown in Sec. IV, are the more reliable ones) cluster around our solution, labeled PY, Eqs. (2.14a) and (2.14b) in Table I. This is as should be expected, since our global solution sums up information from various experimental sets. Third, unlike our global solution, which includes systematic errors, the errors given in Table I for the other fits are only the statistical ones. Finally, we remark that all fits other than the first six have parameters that differ a lot from those obtained fitting only $K$ decay data. Since this last fit is already very good, this makes the lower six fits in Table I suspect. Indeed, and as we will see, they lead to scattering amplitudes that do not satisfy well dispersion relations.

\section{The $I=0 \mathrm{~S}$ wave between $950 \mathrm{MeV}$ and $1420 \mathrm{MeV}$}

The description of pion-pion scattering above the $\bar{K} K$ threshold would imply a full two-channel formalism. To determine the three independent components of the effective range matrix $\Phi, \Phi_{11}, \Phi_{22}$, and $\Phi_{12}$, one requires measurement of three cross sections. Failing this, one gets an indeterminate set, which is reflected very clearly in the wide variations of the effective range matrix parameters in the energy-dependent fits of Protopopescu et al. [10] and Hyams et al. [11(a)].
The raw data themselves are also incompatible; Protopopescu et al. find a phase shift that flattens above $s^{1 / 2} \simeq 1.04 \mathrm{GeV}$, while that of Hyams et al. or Grayer et al. continues to grow. This incompatibility is less marked if we choose the solution with modified higher moments by Protopopescu et al. (Table XIII there). The inelasticities are compatible among the various determinations from $\pi \pi$ scattering, including that of Hyams et al., [11(b)] and Kamiński et al., [11(c)] although the errors of Protopopescu et al. appear to be much underestimated. They are, however, systematically, well above what one finds [11(d)] from $\pi \pi \rightarrow \bar{K} K$ scattering: see Fig. 6 .

We will here give a purely empirical fit, using the $\pi \pi$ data. We write

$$
\begin{gathered}
\cot \delta_{0}^{(0)}(s)=c_{0} \frac{\left(s-M_{s}^{2}\right)\left(M_{f}^{2}-s\right)}{M_{f}^{2} s^{1 / 2}} \frac{\left|k_{2}\right|}{k_{2}^{2}}, \\
k_{2}=\frac{\sqrt{s-4 m_{K}^{2}}}{2} \\
\eta_{0}^{(0)}=1-\left(\epsilon_{1} \frac{k_{2}}{s^{1 / 2}}+\epsilon_{2} \frac{k_{2}^{2}}{s}\right) \frac{M^{\prime 2}-s}{s} ; \\
M^{\prime}=1.5 \mathrm{GeV} \text { (fixed). }
\end{gathered}
$$

The fit to the inelasticity gives

$$
\begin{gathered}
\epsilon_{1}=6.4 \pm 0.5, \quad \epsilon_{2}=-16.8 \pm 1.6 ; \\
\chi^{2} /(\text { d.o.f. })=0.7 .
\end{gathered}
$$

This result is driven by the data of Protopopescu et al., whose accuracy is, unfortunately, much overestimated: see Fig. 6. 


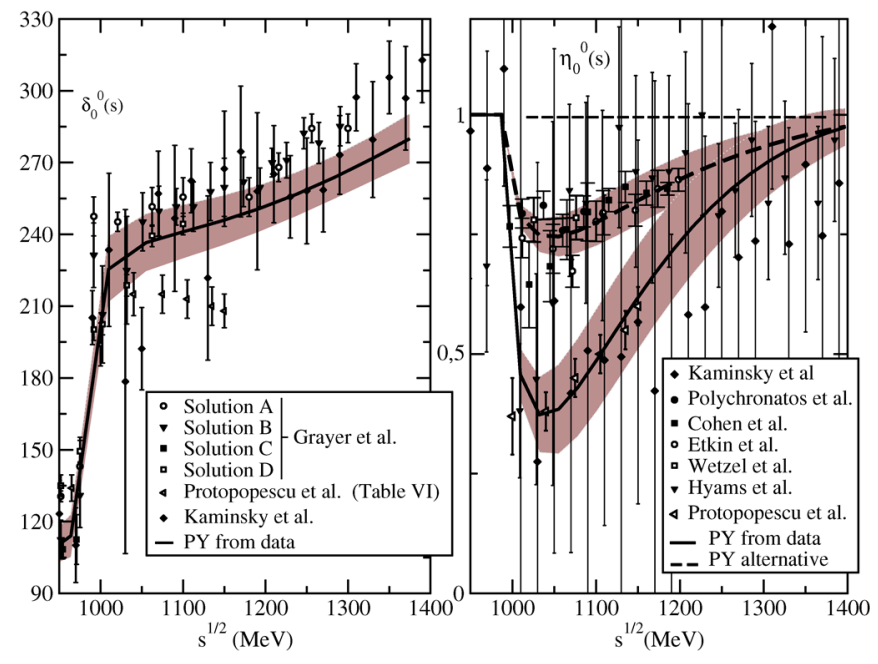

FIG. 6 (color online). Fit to the $I=0, S$-wave inelasticity and phase shift between 950 and $1400 \mathrm{MeV}$. Data from Refs. [10,11]. The difference between the determinations of $\eta_{0}^{(0)}$ from $\pi \pi \rightarrow$ $\pi \pi$ (PY from data) and from $\pi \pi \rightarrow \bar{K} K$ (PY alternative) is apparent here.

If, instead of fitting $\eta_{0}^{(0)}$ to the $\pi \pi$ data of Protopopescu et al. and Hyams et al. we had fitted the data [11(d)] from $\pi \pi \rightarrow \bar{K} K$ scattering (shown in Fig. 6), we would have found values for the $\epsilon_{i}$ much smaller than what was given in $(2.15 b)$ :

$\epsilon_{1}=2.4 \pm 0.2, \quad \epsilon_{2}=-5.5 \pm 0.8 ; \quad \chi^{2} /($ d.o.f. $)=1.3$.

We have checked that the influence of using (2.15b) or $\left(2.15 b^{\prime}\right)$ on the dispersion relations and other evaluations of integrals, to be considered later, is minute, for energies below $0.95 \mathrm{GeV}$. This is because the inelasticity affects little the imaginary part of the partial wave (on the average). Above $1 \mathrm{GeV}$, if we took the $\epsilon_{i}$ following from $\pi \pi \rightarrow K \bar{K}$, Eq. $\left(2.15 \mathrm{~b}^{\prime}\right)$, the dispersion relations would be slightly better fulfilled; see Sec. IVA, at the end. In spite of this, we stick to (2.15b). Taking $\eta_{0}^{(0)}$ from one set of experiments and $\delta_{0}^{(0)}$ from another (incompatible with the first) would be an inconsistent procedure.

To fit $\delta_{0}^{(0)}$ we also require it to agree with the low energy determination we found in Sec. II B 2 at $s^{1 / 2}=0.95 \mathrm{GeV}$. If we include the data of Protopopescu et al. in the fit we find a poor fit with $\chi^{2} /$ d.o.f. $=39 /(14-2)$ and the parameters

$$
\begin{gathered}
c_{0}=1.72 \pm 0.08, \quad M_{s}=920 \mathrm{MeV} \\
M_{f}=1340 \mathrm{MeV} .
\end{gathered}
$$

The error in $c_{0}$, corresponding to $3 \sigma$, is purely nominal. If we keep $M_{s}$ fixed and remove the data of Protopopescu et al., we get a good $\chi^{2} /$ d.o.f., and now

$$
c_{0}=0.79 \pm 0.25, \quad M_{f}=1270 \mathrm{MeV} .
$$

If we want to be compatible with the data of Hyams et al., we must increase the errors. We then take the numbers

$$
\begin{gathered}
c_{0}=1.3 \pm 0.5, \quad M_{f}=1320 \pm 50 \mathrm{MeV}, \\
M_{s}=920 \mathrm{MeV} \text { (fixed) } .
\end{gathered}
$$

The fit is shown in Fig. 6.

We emphasize again that these are purely empirical fits and, moreover, they are fits to data which certainly have uncertainties well beyond their nominal errors, as given in given in (2.15b) and (2.16); something that is obvious for $\eta_{0}^{(0)}$ from Fig. 6. It follows that relations such as dispersion relations in which the $\mathrm{S} 0$ wave plays an important role will be unreliable for energies near and, especially, above $\bar{K} K$ threshold (below these energies, however, both (2.15b) and $\left(2.15 b^{\prime}\right)$ give very similar results). In fact, we will check that mismatches occur when $s^{1 / 2}>0.95 \mathrm{GeV}$. A sound description of the S0 wave for $s^{1 / 2}>0.95 \mathrm{GeV}$ in $\pi \pi$ scattering would require more refined parametrizations and, above all, use of more information than just $\pi \pi$ experimental data, and lies beyond the scope of the present paper.

\section{The D waves}

The D waves cannot be described with the same accuracy as the S, P waves. The data are scanty, and have huge errors. That one can get reasonable fits at all is due to the fact that one can use low energy information from sum rules; specifically, we will impose in the fits the values of the scattering lengths that follow from the Froissart-Gribov representation. Note that this is not circular reasoning, and it only introduces a small correlation: the Froissart-Gribov representation for the D0, D2, F waves depends mostly on the S0, S2 and P waves, and very little on the D0, D2, F waves themselves.

\section{Parametrization of the $I=2 D$ wave}

For isospin equal 2 we would only expect important inelasticity when the channel $\pi \pi \rightarrow \rho \rho$ opens up, so we will take the value $s_{0}=1.45^{2} \mathrm{GeV}^{2} \sim 4 M_{\rho}^{2}$ for the energy at which elasticity is not negligible.

But life is complicated: a pole term is necessary to get an acceptable fit down to low energy since we expect $\delta_{2}^{(2)}$ to change sign near threshold. The experimental measurements (Losty et al.; Hoogland et al. [12]) give negative and small values for the phase above some $500 \mathrm{MeV}$, while chiral perturbation calculations and the Froissart-Gribov representation indicate a positive scattering length [6]. If we want a parametrization that applies down to threshold, we must incorporate this zero of the phase shift. What is more, the clear inflection seen in data around $1 \mathrm{GeV}$ implies that we will have to go to third order in the conformal expansion. So we write 
$\delta_{2}^{(2)}(\mathrm{s})$

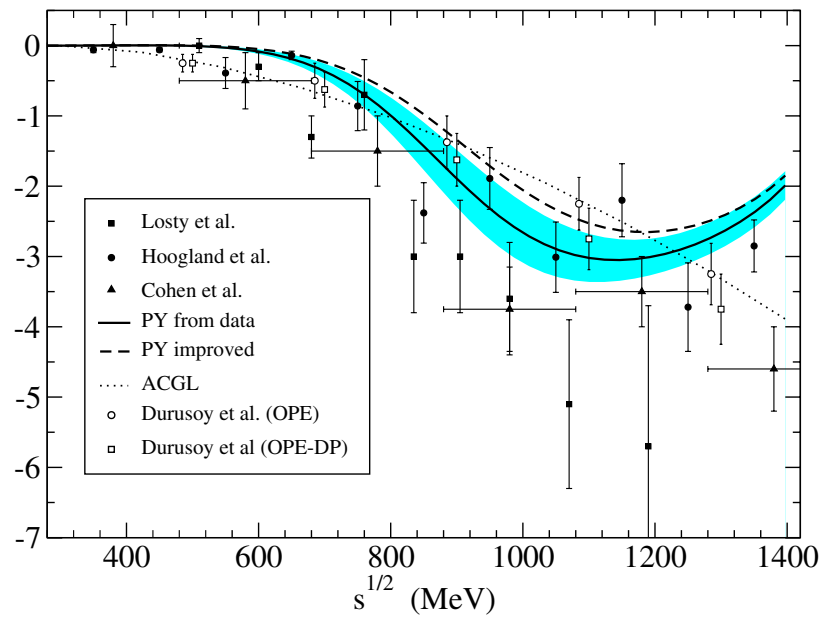

FIG. 7 (color online). Fits to the $I=2, D$-wave phase shift. Continuous line (PY) with Eq. (2.18). Dashed line: PY, after improving with dispersion relations (Sec. IV). Dotted line: the fit used in Refs. [1,2] (ACGL, CGL). Also shown are the data points of Losty et al., from solution A of Hoogland et al. [12], from Cohen et al. [4], and Durusoy et al. [12], the last not included in the fit.

$$
\begin{aligned}
\cot \delta_{2}^{(2)}(s)= & \frac{s^{1 / 2}}{2 k^{5}}\left\{B_{0}+B_{1} w(s)+B_{2} w(s)^{2}\right\} \\
& \times \frac{M_{\pi}^{4} s}{4\left(M_{\pi}^{2}+\Delta^{2}\right)-s}
\end{aligned}
$$

with $\Delta$ a free parameter fixing the zero and

$$
w(s)=\frac{\sqrt{s}-\sqrt{s_{0}-\bar{s}}}{\sqrt{s}+\sqrt{s_{0}-s}}, \quad s_{0}^{1 / 2}=1450 \mathrm{MeV} .
$$

Since the data we have on this wave are not accurate (cf. Fig. 7) we have to include extra information. To be precise, we include in the fit the value of the scattering length that follows from the Froissart-Gribov representation,

$$
a_{2}^{(2)}=(2.78 \pm 0.37) \times 10^{-4} M_{\pi}^{-5},
$$

but not that of the effective range parameter,

$$
b_{2}^{(2)}=(-3.89 \pm 0.28) \times 10^{-4} M_{\pi}^{-7}
$$

(see below, Sec. VI).

We get a mediocre fit, $\chi^{2} /$ d.o.f. $=71 /(25-3)$, and the values of the parameters are

$$
\begin{array}{ll}
B_{0}=(2.4 \pm 0.3) \times 10^{3}, & B_{1}=(7.8 \pm 0.8) \times 10^{3}, \\
B_{2}=(23.7 \pm 3.8) \times 10^{3}, & \Delta=196 \pm 20 \mathrm{MeV} .
\end{array}
$$

We have rescaled the errors by the square root of the $\chi^{2} /$ d.o.f.

The fit, which may be found in Fig. 7, returns reasonable numbers for the scattering length and for the effective range parameter, $b_{2}^{(2)}$ :

$$
\begin{aligned}
& a_{2}^{(2)}=(2.5 \pm 0.9) \times 10^{-4} M_{\pi}^{-5} \\
& b_{2}^{(2)}=(-2.7 \pm 0.8) \times 10^{-4} M_{\pi}^{-7}
\end{aligned}
$$

Although the twist of $\delta_{2}^{(2)}(s)$ at $s^{1 / 2} \sim 1.05 \mathrm{GeV}$ is probably connected to the opening of the $2 \pi \rho$ channel, we neglect the inelasticity of the D2 wave, since it is not detected experimentally. This, together with the incompatibility of the various sets of experimental data and the poor convergence of the conformal series, indicates that the solution given by (2.17a) and (2.17b) is, very likely, somewhat displaced with respect to the "true" D2 wave at the higher energy range (say for $s^{1 / 2} \gtrsim 0.7 \mathrm{GeV}$ ). In fact, the values of the parameters will be improved in Sec. IV with the help of dispersion relations; the D2 phase shift one finds by so doing is slightly displaced with respect to that following from (2.17a) and (2.17b), as shown in Fig. 7.

\section{Parametrization of the $I=0 D$ wave}

The D wave with isospin 0 in $\pi \pi$ scattering presents two resonances below $1.7 \mathrm{GeV}$ : the $f_{2}(1270)$ and the $f_{2}(1525)$, that we will denote, respectively, by $f_{2}, f_{2}^{\prime}$. Experimentally, $\Gamma_{f_{2}}=185 \pm 4 \mathrm{GeV}$ and $\Gamma_{f_{2}^{\prime}}=76 \pm 10 \mathrm{GeV}$. The first, $f_{2}$, couples mostly to $\pi \pi$, with small couplings to $\bar{K} K(4.6 \pm 0.5 \%), 4 \pi(10 \pm 3 \%)$, and $\eta \eta$. The second couples mostly to $\bar{K} K$, with a small coupling to $\eta \eta$ and $2 \pi$, respectively, $10 \pm 3 \%$ and $0.8 \pm 0.2 \%$. This means that the channels $\pi \pi$ and $\bar{K} K$ are essentially decoupled and, to a $15 \%$ accuracy, we may neglect inelasticity up to $s \simeq$ $1.45^{2} \mathrm{GeV}^{2}$.

There are not many experimental data on the D wave which, at accessible energies, is small. So, the compilation of $\delta_{2}^{(0)}$ phase shifts of Protopopescu et al. [10] gives significant numbers for $\delta_{2}^{(0)}$ only in the range $810 \mathrm{MeV} \leq$ $s^{1 / 2} \leq 1150 \mathrm{MeV}$. In view of this, it is impossible to get accurately the D-wave scattering lengths, or indeed any other low energy parameter, from this information: so, we will include information on $a_{2}^{(0)}$ to help stabilize the fits.

We take the data of Protopopescu et al. [10] and consider the so-called "solution 1," with the two possibilities given in their Tables VI and XIII (with modified higher moments), in the range mentioned before, $s^{1 / 2}=0.810 \mathrm{GeV}$ to $1.150 \mathrm{GeV}$. The problem with these data points is that they are certainly biased, as indeed they are quite incompatible with those of other experiments. We can stabilize the fits by fitting also the points ${ }^{3}$ of Estabrooks and Martin, [11(a)] and imposing the value of the width of the $f_{2}$ resonance, with the condition $\Gamma_{f_{2}}=185 \pm 10 \mathrm{MeV}$, as well as the value of the scattering length. We write

${ }^{3}$ For these data we arbitrarily take a common error of $10 \%$. 


$$
\cot \delta_{2}^{(0)}(s)=\frac{s^{1 / 2}}{2 k^{5}}\left(M_{f_{2}}^{2}-s\right) M_{\pi}^{2}\left\{B_{0}+B_{1} w(s)\right\}
$$

and

$$
\begin{gathered}
w(s)=\frac{\sqrt{s}-\sqrt{s_{0}-s}}{\sqrt{s}+\sqrt{s_{0}-s}}, \quad s_{0}^{1 / 2}=1450 \mathrm{MeV} ; \\
M_{f_{2}}=1275.4 \mathrm{MeV} .
\end{gathered}
$$

We find

$$
B_{0}=23.6 \pm 0.7, \quad B_{1}=24.7 \pm 1.0 .
$$

The fit is not good in that we get $\chi^{2} /$ d.o.f. $=300 /(52-$ 2). However, it improves substantially if we exclude the data of Protopopescu et al., which are strongly biased (as is clearly seen in Fig. 8); the central values, however, vary very little. We have included in the errors in (2.19b) half the difference between the two possibilities (with or without the data of Protopopescu et al.)

The fit returns the values

$$
\begin{aligned}
& a_{2}^{(0)}=(18.0 \pm 2.8) \times 10^{-4} M_{\pi}^{-5}, \\
& b_{2}^{(0)}=(-8.1 \pm 3.1) \times 10^{-4} M_{\pi}^{-7} \\
& \Gamma_{f_{2}}=190 \pm 8 \mathrm{MeV} .
\end{aligned}
$$

The value of $b_{2}^{(0)}$ agrees within $1.3 \sigma$ with the more precise result obtained with the Froissart-Gribov representation (see below), that gives

$$
\begin{aligned}
& a_{2}^{(0)}=(18.70 \pm 0.41) \times 10^{-4} M_{\pi}^{-5}, \\
& b_{2}^{(0)}=(-4.16 \pm 0.30) \times 10^{-4} M_{\pi}^{-7} .
\end{aligned}
$$

$\delta_{2}^{(0)}(\mathrm{s})$

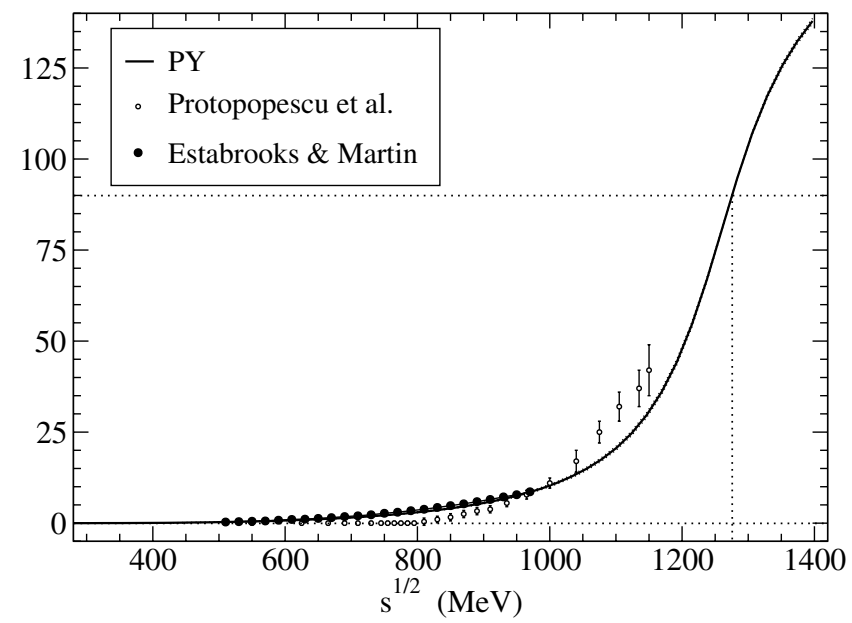

FIG. 8. Fit to the $I=0, D$-wave phase shift. Also shown are the data points from solution 1 of Protopopescu et al. (open circles) and data of Estabrooks and Martin (black dots). The dotted lines mark the $f_{2}(1270)$ resonance, whose location and width were included in the fit.
This agreement is remarkable, taking into account that the D-wave $b_{2}$ calculations are less reliable since they are the ones with a relatively large contribution of the derivative of the $I=2 t$-channel amplitude, which is very uncertain (see Ref. [6]).

We then take into account the inelasticity by writing

$$
\eta_{2}^{(0)}(s)= \begin{cases}1, & s<4 m_{K}^{2}, \\ 1-\epsilon \frac{k_{2}(s)}{k_{2}\left(M_{f_{2}}^{2}\right)}, & \epsilon=0.262 \pm 0.030 ; \quad s>4 m_{K}^{2} ;\end{cases}
$$

$k_{2}=\sqrt{s / 4-m_{K}^{2}}$. We have fixed the coefficient $\epsilon$ fitting the inelasticities of Protopopescu et al. [10], and the experimental inelasticity of the $f_{2}$; the overall $\chi^{2} /$ d.o.f. of this fit is $\sim 1.8$.

In principle, by including $b_{2}^{(0)}$ in our fit the nominal uncertainties in the "fit to data" parameters could be decreased. However, we have not done so, since, as explained before and in Ref. [6], the D-wave $b_{2}$ 's are less reliable than the $a_{2}$ 's (used as input with very large errors) and, therefore, the apparent improvement would be made at the cost of reliability.

\section{The $\mathbf{F}, \mathbf{G}$ waves}

The contribution of the $F$ wave to our sum rules and dispersion relations is very small, but it is interesting to evaluate it (we have included its contribution to all relations) to check, precisely, the convergence of the partial wave series. The contribution of the G0, G2 waves is completely negligible, for the quantities we calculate in our paper, but we describe fits to these for completeness.

\section{The $F$ wave}

The experimental situation for the $\mathrm{F}$ wave is somewhat confused. According to Protopopescu et al. [10], it starts negative (but compatible with zero at the $2 \sigma$ level) and becomes positive around $s^{1 / 2}=1 \mathrm{GeV}$. Hyams et al. [11(a)] report a positive $\delta_{3}(s)$ when it differs from zero (above $s^{1 / 2}=1 \mathrm{GeV}$ ). In both cases no inelasticity is detected up to $s^{1 / 2} \sim 1.4 \mathrm{GeV}$.

The corresponding scattering length may be calculated with the help of the Froissart-Gribov representation and one finds

$$
a_{3}=(6.3 \pm 0.4) \times 10^{-5} M_{\pi}^{-7} .
$$

It could in principle be possible that $\delta_{3}(s)$ changes sign twice, once near threshold and once near $s^{1 / 2}=1 \mathrm{GeV}$. However, we disregard this possibility and write, simply, 


$$
\begin{aligned}
\cot \delta_{3}(s) & =\frac{s^{1 / 2} M_{\pi}^{6}}{2 k^{7}}\left\{B_{0}+B_{1} w(s)\right\}, \\
w(s) & =\frac{\sqrt{s}-\sqrt{s_{0}-s}}{\sqrt{s}+\sqrt{s_{0}-s}}
\end{aligned}
$$

with $s_{0}^{1 / 2}=1.45 \mathrm{GeV}$, and impose the value of $a_{3}$.

It is to be understood that this parametrization provides only an empirical representation of the available data, and that it may not be reliable except at very low energies, where it is dominated by the scattering length, and for $s^{1 / 2} \geq 1 \mathrm{GeV}$. We fit data of Protopopescu et al. [10] for energies above $1 \mathrm{GeV}$, and data of Hyams et al. [11(b)] We have estimated the errors of this last set (not given in the paper) as the distance from the average value to the extreme values in the different solutions given. We find

$$
\begin{gathered}
\frac{\chi^{2}}{\text { d.o.f. }} \simeq \frac{7.7}{14-2}, \quad B_{0}=(1.09 \pm 0.03) \times 10^{5}, \\
B_{1}=(1.41 \pm 0.04) \times 10^{5} .
\end{gathered}
$$

The errors have been increased by including as an error the variation that affects the central values when using only one of the two sets of data. We do not include separately the effects of the $\rho_{3}(1690)$, since its tail is incorporated in the fitted data.

\section{The $G$ waves}

The experimental information on the $G$ waves is very scarce. For the wave G2, we have two nonzero values for $\delta_{4}^{(2)}$ from Cohen et al. [4] and four significant ones from Losty et al. [12]; they are somewhat incompatible. We then fit the data separately, with a one-parameter formula; we write

$$
\cot \delta_{4}^{(2)}(s)=\frac{s^{1 / 2} M_{\pi}^{8}}{2 k^{9}} B .
$$

If we fit the data of Losty et al. we find $B=(-0.56 \pm$ $0.09) \times 10^{6}$, while from Cohen et al. we get $B=$ $(-10.2 \pm 3.0) \times 10^{6}$. Fitting both sets together we find $B=-9.1 \times 10^{6}$, and a very poor $\chi^{2} /$ d.o.f. $=32 /(6-$ 1). Enlarging the resulting error to cover $6 \sigma$, we obtain our best result,

$$
\cot \delta_{4}^{(2)}(s)=\frac{s^{1 / 2} M_{\pi}^{8}}{2 k^{9}} B, \quad B=(-9.1 \pm 3.3) \times 10^{6} .
$$

This formula can only be considered as valid only for a limited range, $0.8 \leq s^{1 / 2} \leq 1.5 \mathrm{GeV}$. In fact, from the Froissart-Gribov representation it follows that the G2 scattering length is positive. One has $a_{4}^{(2)}=(4.5 \pm 0.2) \times$ $10^{-6} M_{\pi}^{-9}$, while (2.24) would give a negative value.

For the G0 wave, the situation is similar. However, we know of the existence of a very inelastic resonance with mass around $2 \mathrm{GeV}$. An effective value for the imaginary part of the corresponding partial wave may be found in Appendix A.

\section{FORWARD DISPERSION RELATIONS}

We expect that the scattering amplitudes that follow from the phase shifts (and inelasticities) at low energy $\left(s^{1 / 2} \leq 1.42 \mathrm{GeV}\right)$, and the Regge expressions at high energy, will satisfy dispersion relations since they fit well the experimental data and are therefore good approximations to the physical scattering amplitudes. In the present section we will check that this is the case, at low energies $\left(s^{1 / 2} \lesssim 0.95 \mathrm{GeV}\right)$, for three independent scattering amplitudes (that form a complete set), which we will conveniently take the following $t$-symmetric or antisymmetric combinations: $\pi^{0} \pi^{0} \rightarrow \pi^{0} \pi^{0}, \pi^{0} \pi^{+} \rightarrow \pi^{0} \pi^{+}$, and the amplitude $I_{t}=1$, corresponding to isospin unity in the $t$ channel. The reason for choosing these amplitudes is that the amplitudes for $\pi^{0} \pi^{0}$ and $\pi^{0} \pi^{+}$depend only on two isospin states, and have positivity properties: their imaginary parts are sums of positive terms. Because of this, the errors are much reduced for them. This is easily verified if we compare the errors in the dispersion relations for $\pi^{0} \pi^{0}$ and $\pi^{0} \pi^{+}$with those for the amplitude with $I_{t}=1$, which depends on three isospin states and has no positivity properties (see below, in Fig. 15).

Here we will not cover the full energy range or try to improve the parameters by requiring fulfillment of the dispersion relations, something that we leave for Sec. IV. We will start discussing the global fit in Sec. II B 2; the results using the individual fits for $\mathrm{S} 0$, as in Sec. II B 3, will be discussed later, in Sec. III C.

In our analysis one should take into account that, for the amplitudes that contain the SO wave, the uncertainties for it above $0.95 \mathrm{GeV}$ induce large errors in the dispersive integrals, and the agreement between dispersive integrals and real parts of the scattering amplitudes evaluated directly becomes affected. This is particularly true for the $\pi^{0} \pi^{0}$ amplitude, dominated by the S0 contribution, where the mismatch becomes important above $\sim 0.7 \mathrm{GeV}$. For the $\pi^{0} \pi^{+}$amplitude, however, since it is not affected by the S0 problem, the fulfillment of the dispersion relation is good, within reasonably small errors, up to the very region where Regge behavior takes over, $s^{1 / 2} \simeq 1.42 \mathrm{GeV}$.

This is a good place to comment on the importance of the contribution of the Regge region (i.e., from energy above $1.42 \mathrm{GeV}$ ) to the various dispersive integrals. Of course, this depends on each dispersion relation. As an indication, we mention that for the unsubtracted dispersion relation (3.7) at threshold, the contribution of the Regge region $(s \geq 1.42 \mathrm{GeV})$ is $9 \%$. This may go up to $23 \%$ around $800 \mathrm{MeV}$. For the subtracted dispersion relation (3.1a) the Regge contribution is of $10 \%$ at $s^{1 / 2}=0.5 \mathrm{GeV}$ and $20 \%$ at $s^{1 / 2}=0.8 \mathrm{GeV}$. It should, however, be noted that, since the estimated uncertainties in the Regge expres- 
sions [5] is $\lesssim 15 \%$, the influence of the uncertainties in our Regge formulas is below the $4 \%$ level up to $\sim 0.8 \mathrm{GeV}$.

A final general comment is that here, as well as for the sum rules that we will discuss in Sec. V and the FroissartGribov calculations (Sec. VI), we only include the contributions of waves up to and including the $F$ wave. We have checked, in a few typical cases, that the contributions of the G0, G2 waves are completely negligible.

\section{A. Even amplitude dispersion relations (with the global fit) \\ 1. $\pi^{0} \pi^{0}$ scattering}

We consider here the forward dispersion relation for $\pi^{0} \pi^{0}$ scattering, subtracted at threshold, $4 M_{\pi}^{2}$. We write, with $F_{00}(s)$ the forward $\pi^{0} \pi^{0}$ amplitude,

$$
\operatorname{Re} F_{00}(s)-F_{00}\left(4 M_{\pi}^{2}\right)=\frac{s\left(s-4 M_{\pi}^{2}\right)}{\pi} \text { P.P. } \int_{4 M_{\pi}^{2}}^{\infty} d s^{\prime} \frac{\left(2 s^{\prime}-4 M_{\pi}^{2}\right) \operatorname{Im} F_{00}\left(s^{\prime}\right)}{s^{\prime}\left(s^{\prime}-s\right)\left(s^{\prime}-4 M_{\pi}^{2}\right)\left(s^{\prime}+s-4 M_{\pi}^{2}\right)} .
$$

In particular, for $s=2 M_{\pi}^{2}$, which will be important when we later discuss the Adler zeros (Sec. IV), we have

$$
F_{00}\left(4 M_{\pi}^{2}\right)=F_{00}\left(2 M_{\pi}^{2}\right)+D_{00}, \quad D_{00}=\frac{8 M_{\pi}^{4}}{\pi} \int_{4 M_{\pi}^{2}}^{\infty} d s \frac{\operatorname{Im} F_{00}(s)}{s\left(s-2 M_{\pi}^{2}\right)\left(s-4 M_{\pi}^{2}\right)} .
$$

We first check the sum rule (3.1b). We take for $F_{00}\left(4 M_{\pi}^{2}\right), F_{00}\left(2 M_{\pi}^{2}\right)$ the values that follow from our fits to experimental data of Sec. II, which provide a representation of partial waves valid below threshold (provided $s>0$ ), and evaluate the dispersive integral with the parametrizations we obtained also in Sec. II. We find fulfillment to less than $1 \sigma$ :

$$
D_{00}=(43 \pm 3) \times 10^{-3}
$$

and

$$
F_{00}\left(4 M_{\pi}^{2}, 0\right)-F_{00}\left(2 M_{\pi}^{2}, 0\right)=(33 \pm 22) \times 10^{-3}
$$

For the difference (which should vanish if the dispersion relation was exactly fulfilled, and which takes into account correlations) we get

$$
F_{00}\left(4 M_{\pi}^{2}, 0\right)-F_{00}\left(2 M_{\pi}^{2}, 0\right)-D_{00}=(-10 \pm 23) \times 10^{-3} .
$$

We can also verify the dispersion relation (3.1a). The result is shown in Fig. 9, where a certain mismatch is observed in some regions. As we will see below, the matching is better for the other dispersion relations because of the smaller weight of the S0 wave there.

$$
\text { 2. } \pi^{0} \pi^{+} \text {scattering }
$$

We have, with $F_{0+}(s)$ the forward $\pi^{0} \pi^{+}$amplitude,

$$
\operatorname{Re} F_{0+}(s)-F_{0+}\left(4 M_{\pi}^{2}\right)=\frac{s\left(s-4 M_{\pi}^{2}\right)}{\pi} \text { P.P. } \int_{4 M_{\pi}^{2}}^{\infty} d s^{\prime} \frac{\left(2 s^{\prime}-4 M_{\pi}^{2}\right) \operatorname{Im} F_{0+}\left(s^{\prime}\right)}{s^{\prime}\left(s^{\prime}-s\right)\left(s^{\prime}-4 M_{\pi}^{2}\right)\left(s^{\prime}+s-4 M_{\pi}^{2}\right)}
$$

In particular, at the point $s=2 M_{\pi}^{2}$, this becomes

$$
F_{0+}\left(4 M_{\pi}^{2}\right)=F_{0+}\left(2 M_{\pi}^{2}\right)+D_{0+}, \quad D_{0+}=\frac{8 M_{\pi}^{4}}{\pi} \int_{8 M_{\pi}^{2}}^{\infty} d s \frac{\operatorname{Im} F_{0+}(s)}{s\left(s-2 M_{\pi}^{2}\right)\left(s-4 M_{\pi}^{2}\right)} .
$$

The calculation is now more precise because $D_{0+}$ is dominated by the $\mathrm{P}$ wave, very well known. We find, for the dispersive evaluation,

$$
D_{0+}=(12 \pm 1) \times 10^{-3} \text {. }
$$

On the other hand, using directly the explicit parametrizations for the partial wave amplitudes we found in Sec. II one has

$$
F_{0+}\left(4 M_{\pi}^{2}, 0\right)-F_{0+}\left(2 M_{\pi}^{2}, 0\right)=(6 \pm 16) \times 10^{-3} .
$$




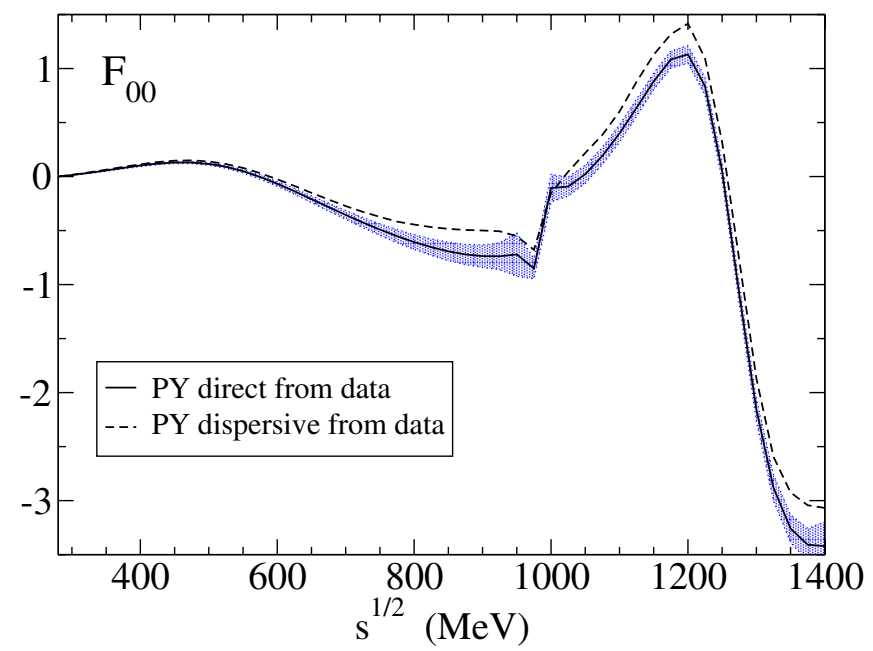

FIG. 9 (color online). The combination $\operatorname{Re} F_{00}(s)-F_{00}\left(4 M_{\pi}^{2}\right)$ (continuous line) and the dispersive integral (broken line).

For the difference,

$F_{0+}\left(4 M_{\pi}^{2}, 0\right)-F_{0+}\left(2 M_{\pi}^{2}, 0\right)-D_{0+}=(-6 \pm 17) \times 10^{-3}$

i.e., perfect agreement.

This is a good place to remark that the agreement of the values of $F_{0+}\left(2 M_{\pi}^{2}, 0\right)$ and $F_{00}\left(2 M_{\pi}^{2}, 0\right)$ obtained with our parametrizations, and those found evaluating dispersion relations [Eqs. (3.2c) and (3.4c)] provides a nontrivial test of the validity of our parametrizations even in regions below threshold, well beyond the region were we fitted data.

The fulfillment of the dispersion relation (3.3a) is shown in Fig. 10 for $s^{1 / 2}$ below $1.4 \mathrm{GeV}$. The agreement is now good in the whole range; the average $\chi^{2} /$ d.o.f. for $s^{1 / 2} \lesssim$

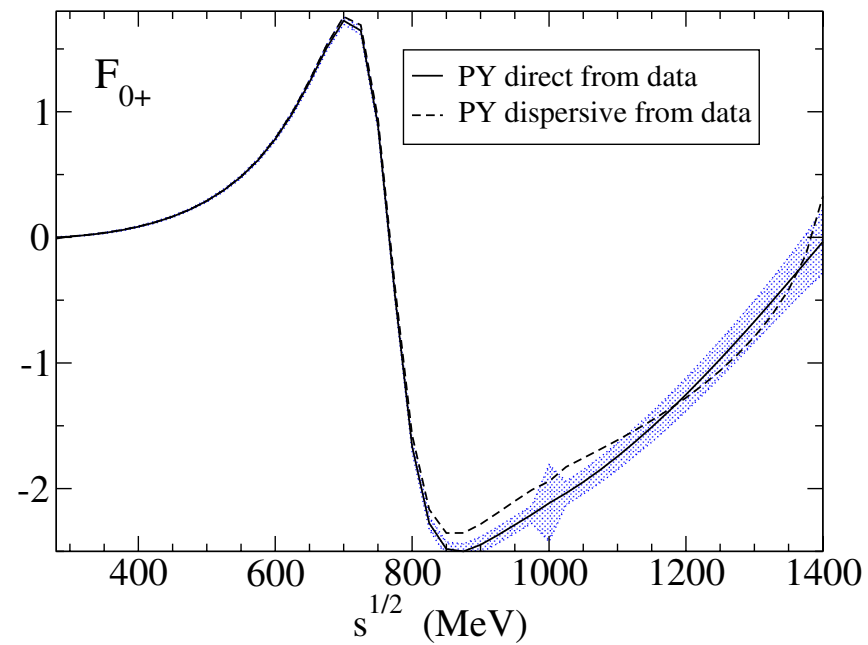

FIG. 10 (color online). The combination $\operatorname{Re} F_{0+}(s)-$ $F_{0+}\left(4 M_{\pi}^{2}\right)$ (continuous line) and the dispersive integral (broken line).
$0.925 \mathrm{GeV}$ is of 1.7. The fact that the fulfillment of the dispersion relation reaches the energy where the Regge formulas start being valid, $s^{1 / 2} \sim 1.4 \mathrm{GeV}$, is yet another test of the consistency of the Regge analysis with the low energy data.

To test the dependence of our results on the point at which we effect the junction between the phase shift analyses and the Regge formulation, we have repeated the calculation of the forward $\pi^{0} \pi^{+}$dispersion relation performing this junction at $1.32 \mathrm{GeV}$, instead of doing so at $1.42 \mathrm{GeV}$. The fulfillment of the dispersion relation improves slightly (below the percent level) at low energy, $s^{1 / 2}<0.75 \mathrm{GeV}$; while it deteriorates a bit more for $s^{1 / 2} \geq$ $0.75 \mathrm{GeV}$. The net results are practically unchanged, with the choice $s^{1 / 2}=1.42 \mathrm{GeV}$ for the junction slightly favored. We will use this number $(1.42 \mathrm{GeV})$ henceforth.

\section{B. The odd amplitude $F^{\left(I_{t}=1\right)}$ dispersion relation and Olsson sum rule (global fit)}

We consider first a forward dispersion relation for the amplitude $F^{\left(I_{t}=1\right)}$ with isospin 1 in the $t$ channel, evaluated at threshold. This is known at times as the (first) Olsson sum rule. Expressing $F^{\left(I_{t}=1\right)}\left(4 M_{\pi}^{2}, 0\right)$ in terms of the scattering lengths, this reads

$$
\begin{aligned}
2 a_{0}^{(0)}-5 a_{0}^{(2)} & =D_{\mathrm{Ol}} \\
D_{\mathrm{Ol}} & \equiv 3 M_{\pi} \int_{4 M_{\pi}^{2}}^{\infty} d s \frac{\operatorname{Im} F^{\left(I_{t}=1\right)}(s, 0)}{s\left(s-4 M_{\pi}^{2}\right)} .
\end{aligned}
$$

In terms of isospin in the $s$ channel,

$$
\begin{aligned}
F^{\left(I_{t}=1\right)}(s, t)= & \frac{1}{3} F^{\left(I_{s}=0\right)}(s, t)+\frac{1}{2} F^{\left(I_{s}=1\right)}(s, t) \\
& -\frac{5}{6} F^{\left(I_{s}=2\right)}(s, t) .
\end{aligned}
$$

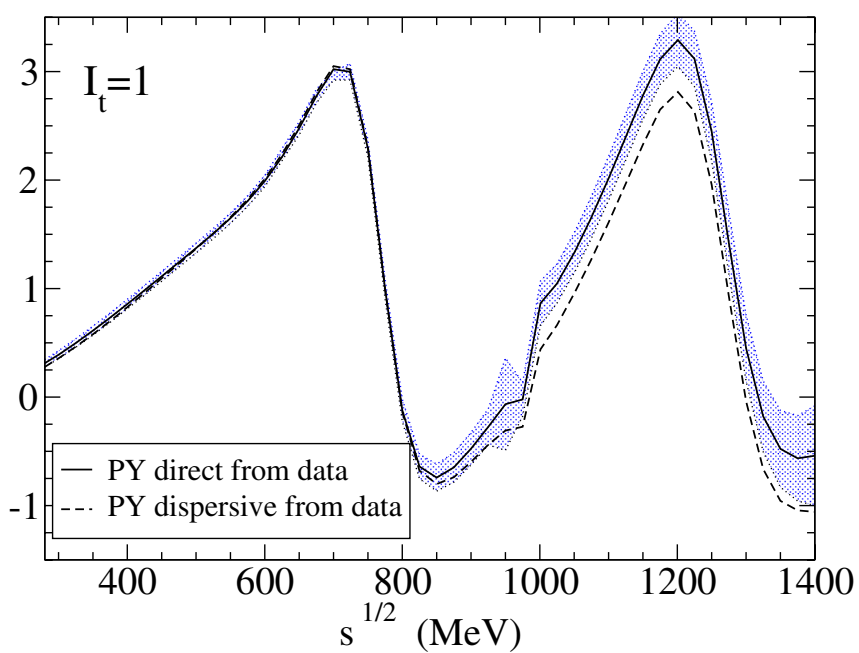

FIG. 11 (color online). The amplitude $\operatorname{Re} F^{I_{t}=1}(s, 0)$ (continuous line) and the dispersive integral (broken line). 
TABLE II. PY, Eqs. (2.14a) and (2.14b): our global fit of Sec. II B 2. The next rows show the fits to K decay [13] alone or combined with $\pi \pi$ scattering data. Grayer A, B, C, E: the solutions in the paper of Grayer et al. [11(a)] EM: the solutions of Estabrooks and Martin [11(a)]. Kaminski refers to the papers of Kamiński et al. [11(c)] Protopopescu VI, XII, and VIII: the corresponding solutions in Ref. [10].

\begin{tabular}{|c|c|c|c|c|c|}
\hline & $B_{0}$ & $B_{1}$ & $\mu_{0}(\mathrm{MeV})$ & $\frac{\chi^{2}}{\text { d.o.f. }}\left(I_{t}=1\right)$ & $\frac{\chi^{2}}{\text { d.o.f. }}\left(\pi^{0} \pi^{0}\right)$ \\
\hline PY, Eqs. (2.14a) and (2.14b) & $21.04^{\mathrm{a}}$ & $6.62^{\mathrm{a}}$ & $782 \pm 24$ & 0.3 & 3.5 \\
\hline$K$ decay only & $18.5 \pm 1.7$ & $\equiv 0$ & $766 \pm 95$ & 0.2 & 1.8 \\
\hline$K$ decay data + Grayer, $\mathrm{B}$ & $22.7 \pm 1.6$ & $12.3 \pm 3.7$ & $858 \pm 15$ & 1.0 & 2.7 \\
\hline$K$ decay data + Grayer, $\mathrm{C}$ & $16.8 \pm 0.85$ & $-0.34 \pm 2.34$ & $787 \pm 9$ & 0.4 & 1.0 \\
\hline$K$ decay data + Grayer, $\mathrm{E}$ & $21.5 \pm 3.6$ & $12.5 \pm 7.6$ & $1084 \pm 110$ & 2.1 & 0.5 \\
\hline$K$ decay data + Kaminski & $27.5 \pm 3.0$ & $21.5 \pm 7.4$ & $789 \pm 18$ & 0.3 & 5.0 \\
\hline$K$ decay data + Grayer, $\mathrm{A}$ & $28.1 \pm 1.1$ & $26.4 \pm 2.8$ & $866 \pm 6$ & 2.0 & 7.9 \\
\hline$K$ decay data + EM, $s$ channel & $29.8 \pm 1.3$ & $25.1 \pm 3.3$ & $811 \pm 7$ & 1.0 & 9.1 \\
\hline$K$ decay data $+\mathrm{EM}, t$ channel & $29.3 \pm 1.4$ & $26.9 \pm 3.4$ & $829 \pm 6$ & 1.2 & 10.1 \\
\hline$K$ decay data + Protopopsecu, VI & $27.0 \pm 1.7$ & $22.0 \pm 4.1$ & $855 \pm 10$ & 1.2 & 5.8 \\
\hline$K$ decay data + Protopopsecu, XII & $25.5 \pm 1.7$ & $18.5 \pm 4.1$ & $866 \pm 14$ & 1.2 & 6.3 \\
\hline$K$ decay data + Protopopsecu, VIII & $27.1 \pm 2.3$ & $23.8 \pm 5.0$ & $913 \pm 18$ & 1.8 & 4.2 \\
\hline
\end{tabular}

${ }^{\mathrm{a}}$ Errors as in Eq. (2.14b).

Substituting in the right hand side above the scattering amplitudes we have just determined up to $1.42 \mathrm{GeV}$, and the Regge expression for rho exchange of Appendix B at higher energies, we find,

$$
D_{\mathrm{Ol}}=0.647 \pm 0.021 \text {. }
$$

(Here, and in all the numbers for scattering lengths and effective ranges, we will take the pion mass $M_{\pi}$ as unity). This is to be compared with what we find from the values of the $a_{0}^{(I)}$ we found in the fits of Sec. II,

$$
2 a_{0}^{(0)}-5 a_{0}^{(2)}=0.719 \pm 0.072 .
$$

For the difference,

$$
2 a_{0}^{(0)}-5 a_{0}^{(2)}-D_{\mathrm{Ol}}=0.073 \pm 0.077,
$$

thus vanishing within errors.

One can also evaluate the corresponding dispersion relation,

$$
\begin{aligned}
\operatorname{Re} F^{\left(I_{t}=1\right)}(s, 0)= & \frac{2 s-4 M_{\pi}^{2}}{\pi} \text { P.P. } \\
& \times \int_{4 M_{\pi}^{2}}^{\infty} d s^{\prime} \frac{\operatorname{Im} F^{\left(I_{t}=1\right)}\left(s^{\prime}, 0\right)}{\left(s^{\prime}-s\right)\left(s^{\prime}+s-4 M_{\pi}^{2}\right)},
\end{aligned}
$$

calculating $\operatorname{Re} F^{\left(I_{t}=1\right)}(s, 0)$ at all values of $s$, either directly using the fits of Sec. II, or from the dispersive integral. The agreement, as shown in Fig. 11, is very good below $1 \mathrm{GeV}$ and reasonably good above this.

\section{Dispersion relations using the individual fits to $\mathbf{S O}$ wave data}

In this subsection we present the results of checking the forward dispersion relations using the individual fits for the S0 wave that we performed in Sec. II B 3. The methods are identical to those used for the solution with the global fit for this wave in the previous subsections, so we will skip details and give only the results, summarized in Table II. Here we give the average $\chi^{4} \chi^{2}$ d.o.f. for the dispersion relations for the amplitudes that contain the S0 wave: $I_{t}=$ 1 and $\pi^{0} \pi^{0}$. The values of the parameters $B_{i}, \mu_{0}$ are, of course, as in Table I, but we repeat them here for ease of reference.

We have separated in Table II the fits which produce a total $\chi^{2} /$ d.o.f. of less than 6 , from those that give a number larger than or equal to 6 when running the corresponding amplitudes through dispersion relations. We may consider that the second set is disfavored by this test. Also, we may repeat some of the comments made in Sec. II B 3 with regard to solution $\mathrm{E}$ of Grayer et al., and the evaluation using $K$ decay data alone: their errors are very large, due of course to the small number of points they fit, so that their fulfillment of dispersion relations is less meaningful than what looks at first sight.

\section{IMPROVING THE PARAMETERS WITH THE HELP OF DISPERSION RELATIONS}

In this section we will show how one can improve the results for the fits to the individual waves that we found in Sec. II: the fact that the dispersion relations are fulfilled with reasonable accuracy at low energy, and that at the $\lesssim 3 \sigma$ level they still hold to higher energies, suggests that we may improve the values of the parameters we have found with our fits to data requiring also better fulfillment of such dispersion relations. This will provide us with a parametrization of the various waves with central values

\footnotetext{
${ }^{4}$ That is to say, the sum of the $\chi^{2}$ of each point, spaced at intervals of $25 \mathrm{MeV}$, divided by the number of points minus the number of free parameters.
} 
more compatible with analyticity and $s-u$ crossing. This method is an alternative to that of the Roy equations to which it is, in principle, inferior in that we do not include $s-t$ crossing (although we check it a posteriori); but it is clearly superior in that we do not need as input the values of the scattering amplitude for $|t|$ up to $30 M_{\pi}^{2}$, where the various Regge fits existing in the literature disagree strongly one with another (see Appendix B) and also in that, with dispersion relations, we can test all energies, whereas the Roy equations are only valid for $s^{1 / 2}<$ $\sqrt{60} M_{\pi} \sim 1.1 \mathrm{GeV}$ and, in practice, only applied up to $0.8 \mathrm{GeV}$.

\section{A. Improved parameters for the global fit of Sec. II B 2}

We will consider the displacement of the central values of the parameters, requiring fulfillment, within errors, of all three dispersion relations for $\sqrt{2} M_{\pi} \leq s^{1 / 2} \leq 0.925 \mathrm{GeV}$ (note that we even go below threshold), starting with the global solution in Eqs. (2.14a) and (2.14b). We do not fit higher energies because we feel that the errors in the input for some waves is too poorly known to give a reliable test there. Specifically, the $\mathrm{P}$ wave in the region $1.15 \mathrm{GeV} \lesssim$ $s^{1 / 2} \lesssim 1.5 \mathrm{GeV}$ is not at all determined by experiment; depending on the fit, a resonance appears, or does not appear, in that region: its mass varies between 1.25 and $1.6 \mathrm{GeV}$. Something similar occurs for the S0 wave above $0.95 \mathrm{GeV}$. Thus, it may well be that the parametrizations we use (which, for example, assume no $\mathrm{P}$ wave resonance below $1.5 \mathrm{GeV}$ ) are biased. Finally, our treatment of the inelasticity of the D0, D2 waves was, of necessity, incomplete. This could likely be the explanation of the slight mismatch of dispersion relations above $1 \mathrm{GeV}$, when the corresponding contributions begin to become important; especially for the real parts of the scattering amplitudes.
Because of this uncertainty with the $\mathrm{P}$ wave above $1.15 \mathrm{GeV}$, and the $\mathrm{S} 0$ wave above $0.95 \mathrm{GeV}$, which goes beyond their nominal errors, and because they are of small importance at low energy, we have not varied the parameters that describe these waves here. We have then minimized the sum of $\chi^{2}$ 's obtained from the variation of the parameters of the waves, within their errors, as obtained from data, plus the average $\chi^{2}$ of the dispersion relations (that we call " $\chi^{2} /$ d.o.f."). This average is obtained evaluating each dispersion relation at intervals of $25 \mathrm{MeV}$ in $s^{1 / 2}$, from threshold up to $s^{1 / 2}=0.925 \mathrm{MeV}$, dividing this by the total number of points. For the dispersion relations for $\pi^{0} \pi^{+}$and $\pi^{0} \pi^{0}$ scattering, we also include in the fit the relations (3.1b) and (3.3b), which are important in fixing the location of the Adler zeros for the S0, S2 waves.

According to this, we allow variation of the parameters of the S0 wave up to $\bar{K} K$ threshold (including the location of the Adler zero, $\left.z_{0}\right)$; the parameters of the $\mathrm{P}$ wave up to $s^{1 / 2}=1.0 \mathrm{GeV}$; and the parameters of S2, D0, D2, and $\mathrm{F}$ waves for all $s^{1 / 2} \leq 1.42 \mathrm{GeV}$. For $\mathrm{S} 2$ we also leave free $z_{2}$. We find that the total variation of the parameters has an average $\chi^{2}$ of 0.38 , showing the remarkable stability of our fits. The only parameters that have varied by $\sim 1 \sigma$ or a bit more are some of the parameters for the S0 and D2 waves. For both, this hardly affects the low energy shape, but alters them a little at medium and higher energies (for D2, see Fig. 7). Given the low quality of experimental data in the two cases, this feature should not be surprising.

As stated above, in the present subsection we take as starting point the $\mathrm{S} 0$ wave we obtained with our global fit in Sec. II B 2. The new central values of the parameters, and the scattering length and effective range parameters (both in units of $M_{\pi}$ ) are listed below.

$$
\begin{aligned}
\mathrm{S} 0 ; s^{1 / 2} \leq 2 m_{K}: B_{0} & =17.4 \pm 0.5 ; B_{1}=4.3 \pm 1.4 ; \mu_{0}=790 \pm 21 \mathrm{MeV} ; z_{0}=195 \mathrm{MeV}[\mathrm{fixed}] \\
a_{0}^{(0)} & =0.230 \pm 0.015 ; b_{0}^{(0)}=0.312 \pm 0.014 . \\
\mathrm{S} 2 ; s^{1 / 2} \leq 1.0: B_{0} & =-80.8 \pm 1.7 ; B_{1}=-77 \pm 5 ; z_{2}=147 \mathrm{MeV}[\mathrm{fixed}] \\
a_{0}^{(2)} & =-0.0480 \pm 0.0046 ; b_{0}^{(2)}=-0.090 \pm 0.006 \\
\mathrm{~S} 2 ; 1.0 \leq s^{1 / 2} \leq 1.42: B_{0} & =-125 \pm 6 ; B_{1}=-119 \pm 14 ; \epsilon=0.17 \pm 0.12 . \\
\mathrm{P} ; s^{1 / 2} \leq 1.05: B_{0} & =1.064 \pm 0.11 ; B_{1}=0.170 \pm 0.040 ; M_{\rho}=773.6 \pm 0.9 \mathrm{MeV} ; \\
a_{1} & =(38.7 \pm 1.0) \times 10^{-3} ; b_{1}=(4.55 \pm 0.21) \times 10^{-3} \\
\mathrm{D} 0 ; s^{1 / 2} \leq 1.42: B_{0} & =23.5 \pm 0.7 ; B_{1}=24.8 \pm 1.0 ; \epsilon=0.262 \pm 0.030 ; \\
a_{2}^{(0)} & =(18.4 \pm 3.0) \times 10^{-4} ; b_{2}^{(0)}=(-8.6 \pm 3.4) \times 10^{-4} \\
\mathrm{D} 2 ; s^{1 / 2} \leq 1.42: B_{0} & =(2.9 \pm 0.2) \times 10^{3} ; B_{1}=(7.3 \pm 0.8) \times 10^{3} ; B_{2}=(25.4 \pm 3.6) \times 10^{3} ; \\
\Delta & =212 \pm 19 \mathrm{MeV} ; a_{2}^{(2)}=(2.4 \pm 0.7) \times 10^{-4} ; b_{2}^{(2)}=(-2.5 \pm 0.6) \times 10^{-4} \\
\mathrm{~F} ; s^{1 / 2} \leq 1.42: B_{0} & =(1.09 \pm 0.03) \times 10^{5} ; B_{1}=(1.41 \pm 0.04) \times 10^{5} ; a_{3}=(7.0 \pm 0.8) \times 10^{-5} \\
{\left[s^{1 / 2}\right.} & \left.\leq 0.925 \mathrm{GeV} ; \text { total average } \chi^{2} / \text { d.o.f. }=0.80\right] .
\end{aligned}
$$


We note that the central values and errors for all the waves, with the exception of the S0 and D2 waves (and a little the S2 wave), are almost unchanged (in some cases, they are unchanged within our precision). The results for the S0, S2, and D2 waves are shown in Figs. 7, 13, and 14.

This brings us to the matter of the errors. In general, one cannot improve much the errors we found in Sec. II by imposing the dispersion relations since, to begin with, they are reasonably well satisfied by our original parametrizations; and, indeed, the errors obtained fitting also the dispersion relations are almost identical to the ones we found in Sec. II. Any improvement would thus be purely nominal and would be marred by the strong correlations among the parameters of the various waves that would be introduced.

The exceptions are, as stated, the S0, S2, and D2 waves. For the first two when including the fulfillment of the dispersion relations in the fits, we have first left the Adler zeros, located at $\frac{1}{2} z_{0}^{2}$ and $2 z_{2}^{2}$, free. We find

$$
z_{0}=195 \pm 21 \mathrm{MeV}, \quad z_{2}=147 \pm 7 \mathrm{MeV} .
$$

Unfortunately, the parameters are now strongly correlated so the small gain obtained would be offset by the complications of dealing with many correlated errors. ${ }^{5}$ Because of this we have fixed the Adler zeros at their central values as given in (4.1b) when evaluating the errors for the other parameters. Then the errors are almost uncorrelated.

For the D2 wave we accept the new errors because its parameters vary by more than $1 \sigma$ from those of Sec. II. Given the poor quality of experimental data, obvious from a look at Fig. 7, we feel justified in trusting more the central values and errors buttressed by fulfillment of dispersion relations.

We consider (4.1a) and (4.1b) to provide the best central values (and some improved errors) for the parameters shown there. The various dispersion relations are fulfilled, up to $s^{1 / 2}=0.925 \mathrm{GeV}$, with an average $\chi^{2}$ of 0.66 (for $\pi^{0} \pi^{0}$ ), of 1.62 for the $\pi^{0} \pi^{+}$dispersion relation, and of 0.40 for the $I_{t}=1$ case. The consistency of our $\pi \pi$ scattering amplitudes that this shows is remarkable, and may be seen depicted graphically for the dispersion relations in Fig. 12, where we show the fulfillment of the dispersion relation up to $s^{1 / 2}=1.42 \mathrm{GeV}$. Mismatch occurs to more than one unity of $\chi^{2} /$ d.o.f. due to the artificial joining of our low and high energy fits to data, to the incomplete treatment of the inelasticity of the D0, D2 waves and, above all, to the uncertainties of the $\mathrm{P}$ and, especially, the S0 wave between 1 and $1.42 \mathrm{GeV}$.

In fact, this mismatch is small; if we take the improved values of the parameters as given in (4.1a), and recalculate

\footnotetext{
${ }^{5}$ There is another reason for not attaching errors to $z_{0}$; the Adler zero is located at $\frac{1}{2} z_{0}^{2} \sim 0.01 \mathrm{GeV}^{2}$, so near the left hand cut that our conformal expansion cannot be considered to be convergent there.
}
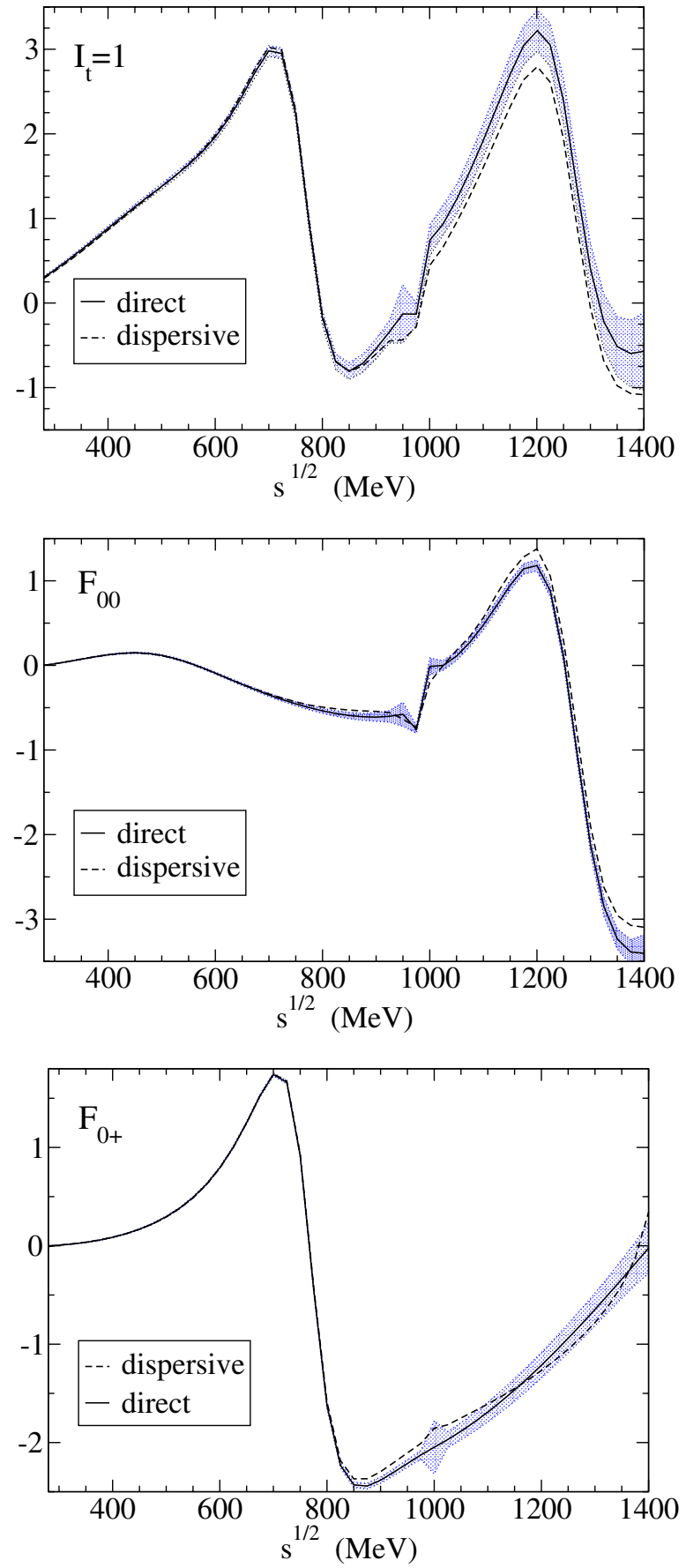

FIG. 12 (color online). Fulfillment of dispersion relations, with the central parameters in (4.1a). The error bands are also shown.

the various dispersion relations up to $s^{1 / 2}=1.42 \mathrm{GeV}$, we find that for $\pi^{0} \pi^{0}$ scattering the dispersion relation is fulfilled with an average $\chi^{2}$ of 1.85 , while for $\pi^{0} \pi^{+}$ scattering we find an average $\chi^{2}$ of 1.57 and the $I_{t}=1$ dispersion relation is fulfilled to an average $\chi^{2}$ of 1.16 . However, the last two numbers become smaller than unity if only we increase the error of the slope of the $\mathrm{P}$ wave between 1 and $1.4 \mathrm{GeV}$ by a factor of 2, i.e., if we take $\lambda_{1}=1.1 \pm 0.4$ in Eq. (2.7). 
Likewise, if we replaced the S0 inelasticity in $(2.15 \mathrm{~b})$ by that in $\left(2.15 b^{\prime}\right)$, the average $\chi^{2}$ for $\pi^{0} \pi^{0}$ would improve to 1.35 , while the average $\chi^{2}$ for the $I_{t}=1$ amplitude would become slightly worse, 1.47. Doubtlessly, the incompatibility of phase and inelasticity for the S0 wave above $1 \mathrm{GeV}$ precludes a perfect fit. We plan, in a coming paper, to use the results here to find consistent values for the phase shift and inelasticity for the S0 and $\mathrm{P}$ waves between 1 and $1.42 \mathrm{GeV}$, by requiring consistency below the $1 \sigma$ level of the dispersion relations in that energy range.

The agreement of dispersion relations up to $1.42 \mathrm{GeV}$ is the more remarkable in that the dispersion relations above $0.925 \mathrm{GeV}$ have not been used to improve any wave.

For the sum rules (3.2a)-(3.2c) and (3.4a)-(3.4c) we now find

$$
\begin{aligned}
& 2 a_{0}^{(0)}-5 a_{0}^{(2)}-D_{\mathrm{Ol}}=(25 \pm 32) \times 10^{-3}, \\
& F_{00}\left(4 M_{\pi}^{2}, 0\right)-F_{00}\left(2 M_{\pi}^{2}, 0\right)-D_{00}=(-15 \pm 9) \times 10^{-3}, \\
& F_{0+}\left(4 M_{\pi}^{2}, 0\right)-F_{0+}\left(2 M_{\pi}^{2}, 0\right)-D_{0+}=(3 \pm 7) \times 10^{-3} .
\end{aligned}
$$

\section{B. Improved parameters for the individual fits of Sec. II B 3}

We now refine the parameters of the fits to data, but taking as starting point the numbers obtained from the individual fits to the various data sets as described in Sec. II B 3. Apart from this, the procedure is identical to that used in Sec. IVA; in particular, we also fit the relations (3.1b) and (3.3b). The results of the evaluations are presented in Table III, where we include the solution (4.1a) and (4.1b), and also what we find if requiring the fit to only $K$ decay data for the S0, as given in Eq. (2.12).

The following comments are in order. First of all, we have the remarkable convergence of the first three solutions in Table III; and even the last three solutions approach our evaluation, PY, Eqs. (4.1a) and (4.1b). This convergence is not limited to the S0 wave: the parameters for all waves other than the S0 agree, within $\lesssim 1 \sigma$, for all solutions in Table III, with the numbers given for the PY solution in Eqs. (4.1a) and (4.1b). For this reason we do not give in detail, for each individual fit, the improved solutions for waves other than the S0 wave. Of course, the values of the $\chi^{2}$, given in Table III have been evaluated with the corresponding improved solutions for all waves.

Second, the fulfillment of dispersion relations is similar for all solutions in Table III. However, the solutions based on Grayer B, Grayer E, and that based on the data of Kamiński et al., fail to satisfy the sum rule (3.1b) by a large amount even though they have large errors; see the last column in Table III. We should, therefore, consider the three solutions PY, that based on $K$ decay data only, and the solution based on Grayer $\mathrm{C}$, to be clearly favored by this consistency test. This is satisfactory in that the solution $\mathrm{C}$ was obtained by Grayer et al. [11] from solution B by including absorption corrections.

Third, we may consider the solution based on $K$ decay data only, and with a single parameter $B_{0}$, to be in the nature of a first approximation, and the introduction of the parameter $B_{1}$ as producing the more accurate solutions denoted by Grayer $C$ and PY. In this respect, the coincidence of the common parameters of the first three solutions in Table III within $\lesssim 2 \sigma$ is satisfactory. And, indeed, this coincidence is even more pronounced. In Fig. 13 we show our starting, global fit from Eqs. (2.14a) and (2.14b) together with the improved solution PY, Eqs. (4.1a) and (4.1b) and the improved solution corresponding to Grayer C: they are all three contained inside the error band of PY, Eqs. (4.1a) and (4.1b), with which the solution Grayer C practically overlaps. We also show, in Fig. 14, the S2 wave, before and after improvement.

Finally, and in spite of the virtual coincidence between the two improved solutions Grayer C and PY, Eqs. (4.1a) and (4.1b), we consider the last to be preferred: it incorporates data from various experiments and is thus less likely to be biased by systematic errors. Because of all this, we feel confident in considering our solution in Eqs. (4.1a) and (4.1b) to be fully validated, and the method we have used to be well tested. We will then henceforth accept and work

TABLE III. PY, Eqs. (4.1a) and (4.1b): our global fit, improved with forward dispersion relations, as explained in Sec. IVA. Grayer A, B, C, E means that we take, as experimental low energy data for the S0 wave, the solutions in Grayer et al. [11(a)] fitted as shown in Table I. Kaminski means we have used the data of Kamiński et al. [11(c)] In these fits we have improved the parameters requiring fulfillment of dispersion relations. Although errors are given for the Adler zero, we have fixed it at its central value when evaluating

\begin{tabular}{|c|c|c|c|c|c|c|c|c|}
\hline Improved fits: & $B_{0}$ & $B_{1}$ & $\mu_{0}(\mathrm{MeV})$ & $z_{0}(\mathrm{MeV})$ & $\frac{\chi^{2}\left(I_{t}=1\right)}{\text { d.o.f. }}$ & $\frac{\chi^{2}\left(\pi^{0} \pi^{0}\right)}{\text { d.o.f. }}$ & $\frac{\chi^{2}\left(\pi^{0} \pi^{+}\right)}{\text {d.o.f. }}$ & $(3.1 \mathrm{~b})$ \\
\hline PY, Eqs. (4.1a) and (4.1b) & $17.4 \pm 0.5$ & $4.3 \pm 1.4$ & $790 \pm 21$ & $198 \pm 21$ & 0.40 & 0.66 & 1.62 & $1.6 \sigma$ \\
\hline$K$ decay only & $16.4 \pm 0.9$ & $\equiv 0$ & $809 \pm 53$ & $182 \pm 34$ & 0.30 & 0.29 & 1.77 & $1.5 \sigma$ \\
\hline$K$ decay data + Grayer, $\mathrm{C}$ & $16.2 \pm 0.7$ & $0.5 \pm 1.8$ & $788 \pm 9$ & $184 \pm 39$ & 0.37 & 0.32 & 1.74 & $1.5 \sigma$ \\
\hline$K$ decay data + Grayer, $\mathrm{B}$ & $20.7 \pm 1.0$ & $11.6 \pm 2.6$ & $861 \pm 14$ & $233 \pm 30$ & 0.37 & 0.83 & 1.6 & $4.0 \sigma$ \\
\hline$K$ decay data + Grayer, $\mathrm{E}$ & $20.2 \pm 2.2$ & $8.4 \pm 5.2$ & $982 \pm 95$ & $272 \pm 50$ & 0.60 & 0.09 & 1.4 & $6.0 \sigma$ \\
\hline$K$ decay data + Kaminski & $20.8 \pm 1.4$ & $13.6 \pm 3.7$ & $798 \pm 17$ & $245 \pm 39$ & 0.43 & 1.08 & 1.36 & $4.5 \sigma$ \\
\hline
\end{tabular}
other errors. We have included the fulfillment of the sum rule (3.1b) in the last column, but not (3.3b) and (3.5), which are verified within $1 \sigma$ by all solutions. 


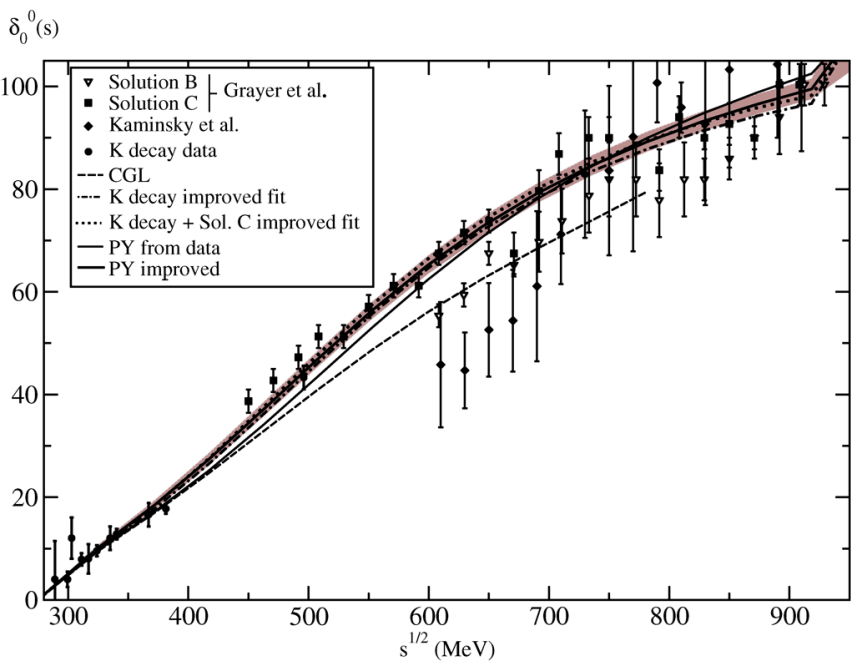

FIG. 13 (color online). The S0 phase shift corresponding to Eqs. (4.1a) and (4.1b) (PY, thick continuous line and error band), the unimproved solution of Eqs. (2.14a) and (2.14b) (thin continuous line), and the improved solutions " $K$ decay only" and "Grayer C" of Table III (difficult to see as they fall almost on top of PY). The solution CGL [2] (lowest discontinuous line) is also shown.

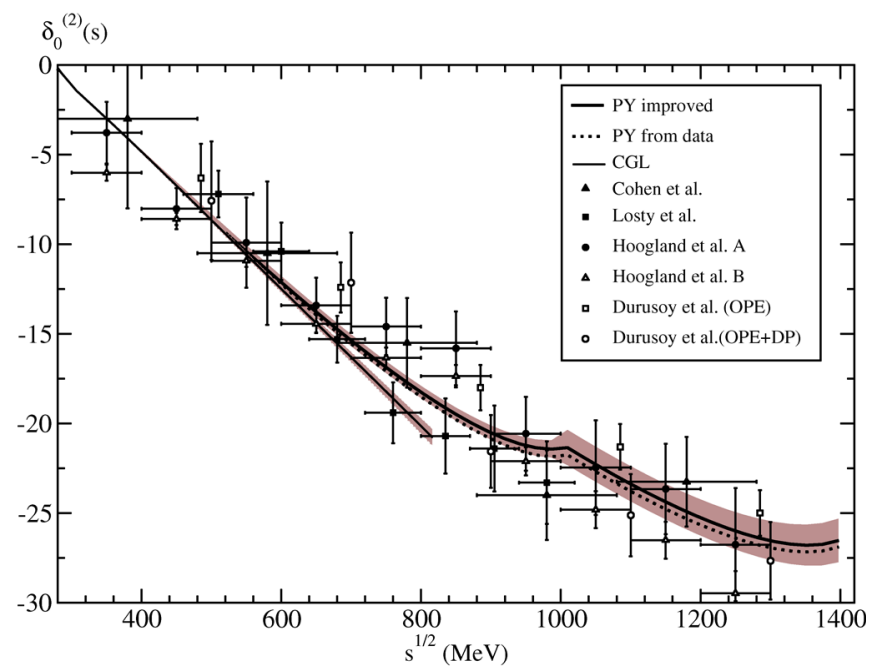

FIG. 14 (color online). Phase shift for the S2 wave, Eqs. (4.1a) and (4.1b) (PY, thick continuous line, and error band), our unimproved fit, Eq. (2.8a) and (2.8b) (dotted line) and the solution CGL, [2] and error band (dashed line). We also show the data from Durusoy et al. [12] and solution B in Hoogland et al. [12], not included in the fit.

with the solution given in Eqs. (4.1a) and (4.1b) for the improved S0 wave below $0.95 \mathrm{GeV}$.

\section{CONSISTENCY TESTS OF $s-t-u$ CROSSING: TWO SUM RULES}

In this section we discuss two sum rules that follow from crossing symmetry; in the next section we will consider Froissart-Gribov sum rules, which can also be viewed as checks of $s-t-u$ crossing. The sum rules we discuss now relate high $\left(s^{1 / 2} \geq 1.42 \mathrm{GeV}\right)$ and low energy, with the low energy given by the $\mathrm{P}, \mathrm{D}, \mathrm{F}$ waves in the region $s^{1 / 2} \leq 1.42 \mathrm{GeV}$ and the high energy is dominated by, respectively, the rho and Pomeron Regge trajectories. The interest in checking them is that they were given by the authors in Refs. [1,2] (following Pennington [15]) as the reason for the incorrect Regge parameters they used. Contrarily to the assertions in these references, however, we will here check, once again, that there is perfect consistency provided one uses standard Regge behavior for energies above $1.42 \mathrm{GeV}$, and accurate representations of the experimental partial waves below $1.42 \mathrm{GeV}$, such as the ones found here in Secs. II and IV and collected in Appendix A. We should perhaps remark here that the contributions of the S0, S2 waves cancel in both sum rules, hence we do not even need to worry about which solution to use for the S0 wave.

The first sum rule is obtained by profiting from the threshold behavior to write an unsubtracted forward dispersion relation for the quantity $F^{\left(I_{s}=1\right)}(s, 0) /\left(s-4 M_{\pi}^{2}\right)$. One gets

$$
\begin{aligned}
\frac{6 M_{\pi}}{\pi} a_{1}= & \frac{1}{\pi} \int_{M_{\pi}^{2}}^{\infty} d s \frac{\operatorname{Im} F^{\left(I_{s}=1\right)}(s, 0)}{\left(s-4 M_{\pi}^{2}\right)^{2}} \\
& +\frac{1}{\pi} \sum_{I} C_{1 I}^{(s u)} \int_{M_{\pi}^{2}}^{\infty} d s \frac{\operatorname{Im} F^{(I)}(s, 0)}{s^{2}},
\end{aligned}
$$

which is known at times as the (second) Olsson sum rule; see e.g. the textbook of Martin et al. [16]. The index $I$ refers to isospin in the $s$ channel and $C_{1 I}^{(s u)}$ are the $s-u$ crossing matrix elements. Canceling $a_{1}$ with the FroissartGribov expression for this quantity (cf. Sec. VI), and substituting the $C_{1 I}^{(s u)}$, we find the result

$$
\begin{aligned}
I \equiv & \int_{M_{\pi}^{2}}^{\infty} d s \frac{\operatorname{Im} F^{\left(I_{t}=1\right)}\left(s, 4 M_{\pi}^{2}\right)-\operatorname{Im} F^{\left(I_{t}=1\right)}(s, 0)}{s^{2}} \\
& -\int_{M_{\pi}^{2}}^{\infty} d s \frac{8 M_{\pi}^{2}\left[s-2 M_{\pi}^{2}\right]}{s^{2}\left(s-4 M_{\pi}^{2}\right)^{2}} \operatorname{Im} F^{\left(I_{s}=1\right)}(s, 0)=0 .
\end{aligned}
$$

The contributions of the $S$ waves cancel in (5.2), so only the $\mathrm{P}, \mathrm{D}, \mathrm{F}$, and $\mathrm{G}$ waves contribute. At high energy the integrals are dominated by rho exchange. We take the improved central values of the parameters for the different waves as given in Eqs. (4.1a) and (4.1b). In units of the pion mass we get

$$
I=(-0.12 \pm 1.27) \times 10^{-5},
$$

that is to say, perfect consistency.

The second sum rule we discuss is that given in Eqs. (B.6), (B.7) of Ref. [1]. It reads, 


$$
\begin{aligned}
J & \equiv \int_{4 M_{\pi}^{2}}^{\infty} d s\left\{\frac{4 \operatorname{Im} F^{\prime(0)}(s, 0)-10 \operatorname{Im} F^{\prime(2)}(s, 0)}{s^{2}\left(s-4 M_{\pi}^{2}\right)^{2}}\right. \\
& \left.-6\left(3 s-4 M_{\pi}^{2}\right) \frac{\operatorname{Im} F^{\prime(1)}(s, 0)-\operatorname{Im} F^{(1)}(s, 0)}{s^{2}\left(s-4 M_{\pi}^{2}\right)^{3}}\right\}=0 .
\end{aligned}
$$

Here $F^{\prime(I)}(s, t)=\partial F^{(I)}(s, t) / \partial \cos \theta$, and the upper indices refer to isospin in the $s$ channel. We get, again with the improved parameters and with $M_{\pi}=1$,

$$
J=(-0.2 \pm 4.2) \times 10^{-3} .
$$

These are not the only crossing sum rules that our $\pi \pi$ amplitude verifies; the coincidence of the values for the parameters $a_{1}, b_{1}, b_{2}^{(I)}$ obtained from direct fits to data in Sec. II with those from the Froissart-Gribov projection, that involves simultaneously $s, u$ and $t$ crossing, are highly nontrivial ones. We will see this in next section.

\section{LOW ENERGY PARAMETERS FOR WAVES WITH $l \geq 1$ FROM THE FROISSART-GRIBOV PROJECTION, AND A NEW SUM RULE FOR $b_{1}$}

\section{A. The Froissart-Gribov representation for $a_{1}, b_{1}$}

The quantities $a_{1}, b_{1}$ may be evaluated in terms of the $I_{t}=1$ amplitude using the Froissart-Gribov representation (for more details on the Froissart-Gribov representation, see Refs. [6,17] and work quoted there). For, e.g. $a_{l}$ with $l=$ odd, we have

$$
2 a_{l}^{(I=1)}=\frac{\sqrt{\pi} \Gamma(l+1)}{4 M_{\pi} \Gamma(l+3 / 2)} \int_{4 M_{\pi}^{2}}^{\infty} d s \frac{\operatorname{Im} F^{\left(I_{t}=1\right)}\left(s, 4 M_{\pi}^{2}\right)}{s^{l+1}} .
$$

We will use the parametrizations in (4.1a) and (4.1b) for the imaginary parts of the scattering amplitudes in all the Froissart-Gribov integrals, and the Regge expressions in Appendix B here at high energy. We find the following results, in units of $M_{\pi}$ :

$$
\begin{array}{ccc}
\text { F.-G. } & \text { Eqs. (4.1a, 4.1b) } \\
10^{3} \times a_{1}: & 37.7 \pm 1.3 & 38.7 \pm 1.0 .
\end{array}
$$

We here compare the result obtained with (6.1), denoted by F.-G., and the value for $a_{1}$ which we found in our improved fits, as given in Eq. (4.1a). Because the two determinations are essentially independent, we can compose the errors to get a precise and reliable value for $a_{1}$ :

$$
a_{1}=(38.4 \pm 0.8) \times 10^{-3} M_{\pi}^{-3} \text {. }
$$

For the quantity $b_{1}$ we have, with the same conventions as before,

$$
\begin{array}{ccc} 
& \text { F.-G. } & \text { Eqs. (4.1a, 4.1b) } \\
10^{3} \times b_{1}: & 4.69 \pm 0.98 & 4.55 \pm 0.21
\end{array}
$$

The Froissart-Gribov integral here is dominated by the rho Regge pole, as the low energy contributions cancel almost completely. The agreement between these two determina- tions of $b_{1}$ is, therefore, a highly nontrivial test of the consistency of the high and low energy parts of our pionpion scattering amplitude, unfortunately not very precise because of the large error in the Froissart-Gribov determination. We will discuss this further at the end of Appendix B.

The $\mathrm{F}$ wave scattering length may be similarly evaluated; we find

$$
a_{3}=(6.3 \pm 0.4) \times 10^{-5} M_{\pi}^{-7} .
$$

\section{B. The Froissart-Gribov projection for even amplitudes: the $a_{2}^{(I)}, b_{2}^{(I)}$ parameters}

We first calculate the two combinations of scattering lengths $\quad a(0+)=\frac{2}{3}\left[a_{2}^{(0)}-a_{2}^{(2)}\right] \quad$ and $\quad a(00)=\frac{2}{3} \times$ $\left[a_{2}^{(0)}+2 a_{2}^{(2)}\right]$. They correspond to the $s$-channel amplitudes

$$
\begin{aligned}
F_{\pi^{0} \pi^{+}} & =\frac{1}{2} F^{\left(I_{s}=1\right)}+\frac{1}{2} F^{\left(I_{s}=2\right)} \\
F_{\pi^{0} \pi^{0}} & =\frac{1}{3} F^{\left(I_{s}=0\right)}+\frac{2}{3} F^{\left(I_{s}=2\right)}
\end{aligned}
$$

for which (as mentioned before) errors are minimized.

The dominant high energy part in the Froissart-Gribov representation is given now by the Pomeranchuk trajectory and its importance is smaller than previously because the integrals converge faster. We find, in units of $M_{\pi}$,

$$
\begin{aligned}
& a(0+)=(10.61 \pm 0.14) \times 10^{-4} M_{\pi}^{-5} \\
& a(00)=(16.17 \pm 0.75) \times 10^{-4} M_{\pi}^{-5} .
\end{aligned}
$$

For the effective range parameters,

$$
\begin{gathered}
b(0+)=(-0.183 \pm 0.061) \times 10^{-4} M_{\pi}^{-7} ; \\
b(00)=(-7.96 \pm 0.57) \times 10^{-4} M_{\pi}^{-7} .
\end{gathered}
$$

\section{A new sum rule for $b_{1}$}

The Froissart-Gribov representation for the effective range $b_{1}$ depends strongly on the Regge parameters for rho exchange, and is affected by large errors. Here we will present a sum rule that, contrarily, depends almost entirely on the low energy scattering amplitudes and is much more precise. It is obtained in a way similar to that used for the first crossing sum rule in Sec. V. We now write a dispersion relation for the quantity

$$
\frac{\partial}{\partial s}\left(\frac{F^{\left(I_{s}=1\right)}(s, 0)}{s-4 M_{\pi}^{2}}\right)
$$

which we evaluate at threshold. Taking into account that

$$
\frac{\partial}{\partial s}\left(\frac{F^{\left(I_{s}=1\right)}(s, 0)}{s-4 M_{\pi}^{2}}\right)_{s=4 M_{\pi}^{2}}=\frac{3 M_{\pi}}{2 \pi} b_{1},
$$

we obtain a fastly convergent relation for $b_{1}$ : 


$$
\begin{aligned}
M_{\pi} b_{1}= & \frac{2}{3} \int_{4 M_{\pi}^{2}}^{\infty} d s\left\{\frac{1}{3}\left[\frac{1}{\left(s-4 M_{\pi}^{2}\right)^{3}}-\frac{1}{s^{3}}\right] \operatorname{Im} F^{\left(I_{t}=0\right)}(s, 0)\right. \\
& +\frac{1}{2}\left[\frac{1}{\left(s-4 M_{\pi}^{2}\right)^{3}}+\frac{1}{s^{3}}\right] \operatorname{Im} F^{\left(I_{t}=1\right)}(s, 0) \\
& \left.-\frac{5}{6}\left[\frac{1}{\left(s-4 M_{\pi}^{2}\right)^{3}}-\frac{1}{s^{3}}\right] \operatorname{Im} F^{\left(I_{t}=2\right)}(s, 0)\right\}
\end{aligned}
$$

Most of the contribution to $b_{1}$ comes from the S0 and $\mathrm{P}$ waves at low energy, while all other contributions (in particular, the Regge contributions) are substantially smaller than $10^{-3}$. Adding all pieces we find

$$
b_{1}=(4.99 \pm 0.21) \times 10^{-3} M_{\pi}^{-5},
$$

a value reasonably compatible with what we found in (4.1a) and (4.1b), $b_{1}=(4.55 \pm 0.21) \times 10^{-3} M_{\pi}^{-5}$ (because of correlations, the distance is actually below $1 \sigma$ ). We can combine both and find a precise estimate,

$$
b_{1}=(4.75 \pm 0.16) \times 10^{-3} M_{\pi}^{-5} .
$$

\section{COMPARISON WITH THE LOW ENERGY PARAMETERS OF CGL, DFGS, AND KLL}

We present in Table IV a global comparison of the low energy parameters as given here, that we denote by PY, as well as recent evaluations, that use the Roy equations, by Colangelo, Gasser, and Leutwyler [2] (CGL), by Descotes et al. [8], that we denote by DFGS, and by Kamiński, Leśniak, and Loiseau [8], denoted by KLL. Besides scattering lengths and effective range parameters, we give the quantities $\left[a_{0}^{(0)}-a_{0}^{(2)}\right]^{2}$ and $\delta_{0}^{(0)}\left(m_{K}^{2}\right)-\delta_{0}^{(2)}\left(m_{K}^{2}\right)$, relevant, respectively, for pionic atom decays and $C P$ violating kaon decays.

The DFGS solution is compatible, within its errors, both with CGL and PY. As to the KLL solution, its values for $a_{0}^{(I)}, b_{0}^{(I)}$ are compatible with those of DFGS (for the first), somewhat less so with the numbers of PY and with what CGL find. The central value for $a_{1}$ of KLL is too high, although it is compatible within its errors with other determinations. The value of $b_{1}$, however, is $3 \sigma$ below the next lowest one in the Table IV. The reason could be that Kamiński, Leśniak, and Loiseau use some approximate calculation techniques, such as effective, separable potentials and Padé approximants.

The calculation of CGL [2] is different from the others, since CGL impose ch.p.t. to two loops. However, CGL use Roy equations, which require phenomenological input for energies above $0.8 \mathrm{GeV}$. As stated in the Introduction (and discussed in more detail in Refs. [6,7]) some of this input is not accurate, which may cause biases in the final results. In this respect, we find that the predictions of CGL for the scattering lengths $a_{0}^{(I)}, a_{1}$ agree very well with what we find from experiment. This means that our results do not challenge the standard chiral counting of ch.p.t. However, the solution given by CGL deviates from our results for quantities like $b_{1}, a_{2}^{(I)}, b_{2}^{(I)}$, or the $\mathrm{S} 0$ and $\mathrm{S} 2$ phase shifts above $500 \mathrm{MeV}$ [in particular we disagree by $3 \sigma$ with the value used as input by CGL at the matching point, $\left.\delta_{0}^{(0)}\left((800 \mathrm{MeV})^{2}\right)=82.3 \pm 3.4^{\circ}\right]$.

TABLE IV. Units of $M_{\pi}$. The numbers in the CGL column are as given by CGL in Table II and elsewhere in their text. In PY, the values for the D, F waves parameters are from the Froissart-Gribov representation. The rest are from the fits, improved with dispersion relations, except for $a_{1}$ and $b_{1}$ that have been taken from Eqs. (6.3) and (6.10).

\begin{tabular}{lcccc}
\hline \hline & DFGS & KLL & CGL & PY \\
\hline$a_{0}^{(0)}$ & $0.228 \pm 0.032$ & $0.224 \pm 0.013$ & $0.220 \pm 0.005$ & $0.230 \pm 0.015$ \\
$a_{0}^{(2)}$ & $-0.0382 \pm 0.0038$ & $-0.0343 \pm 0.0036$ & $-0.0444 \pm 0.0010$ & $-0.0480 \pm 0.0046$ \\
{$\left[a_{0}^{(0)}-a_{0}^{(2)}\right]^{2}$} & $0.071 \pm 0.018$ & $0.067 \pm 0.007$ & $0.070 \pm 0.003$ & $0.077 \pm 0.008$ \\
$\delta_{0}^{(0)}\left(m_{K}^{2}\right)-\delta_{0}^{(2)}\left(m_{K}^{2}\right)$ & & $47.7 \pm 1.5^{\circ}$ & $52.9 \pm 1.6^{\circ}$ \\
$b_{0}^{(0)}$ & & $0.252 \pm 0.011$ & $0.280 \pm 0.001$ & $0.312 \pm 0.014$ \\
$b_{0}^{(2)}$ & & $-0.075 \pm 0.015$ & $-0.080 \pm 0.001$ & $-0.090 \pm 0.006$ \\
$a_{1}$ & $39.6 \pm 2.4$ & $37.9 \pm 0.5$ & $38.4 \pm 0.8\left(\times 10^{-3}\right)$ \\
$b_{1}$ & $2.83 \pm 0.67$ & $5.67 \pm 0.13$ & $4.75 \pm 0.16\left(\times 10^{-3}\right)$ \\
$a_{2}^{(0)}$ & & $17.5 \pm 0.3$ & $18.70 \pm 0.41\left(\times 10^{-4}\right)$ \\
$a_{2}^{(2)}$ & & $1.70 \pm 0.13$ & $2.78 \pm 0.37\left(\times 10^{-4}\right)$ \\
$a(0+)$ & & & $10.61 \pm 0.14\left(\times 10^{-4}\right)$ \\
$a(00)$ & & $-3.55 \pm 0.14$ & $16.17 \pm 0.75\left(\times 10^{-4}\right)$ \\
$b_{2}^{(0)}$ & & $-3.26 \pm 0.12$ & $-3.89 \pm 0.28\left(\times 10^{-4}\right)$ \\
$b_{2}^{(2)}$ & & & $-0.183 \pm 0.061\left(\times 10^{-4}\right)$ \\
$b(0+)$ & & & $-7.96 \pm 0.57\left(\times 10^{-4}\right)$ \\
$b(00)$ & & $5.6 \pm 0.2$ & $6.3 \pm 0.4\left(\times 10^{-5}\right)$ \\
$a_{3}$ & & & & \\
\hline \hline
\end{tabular}


That the CGL solution deteriorates as the energy increases is quite transparent if we compare the fulfillment of dispersion relations with the parameters of CGL for the S0, S2, and P waves at low energy, or with our parameters here. This is depicted in Fig. 15, where we show the mismatch between the real part and the dispersive evaluations, that is to say, the differences $\Delta_{i}$,

$$
\begin{gathered}
\Delta_{1} \equiv \operatorname{Re} F^{\left(I_{t}=1\right)}(s, 0)-\frac{2 s-4 M_{\pi}^{2}}{\pi} \text { P.P. } \int_{4 M_{\pi}^{2}}^{\infty} d s^{\prime} \frac{\operatorname{Im} F^{\left(I_{t}=1\right)}\left(s^{\prime}, 0\right)}{\left(s^{\prime}-s\right)\left(s^{\prime}+s-4 M_{\pi}^{2}\right)}, \\
\Delta_{00} \equiv \operatorname{Re} F_{00}(s)-F_{00}\left(4 M_{\pi}^{2}\right)-\frac{s\left(s-4 M_{\pi}^{2}\right)}{\pi} \text { P.P. } \int_{4 M_{\pi}^{2}}^{\infty} d s^{\prime} \frac{\left(2 s^{\prime}-4 M_{\pi}^{2}\right) \operatorname{Im} F_{00}\left(s^{\prime}\right)}{s^{\prime}\left(s^{\prime}-s\right)\left(s^{\prime}-4 M_{\pi}^{2}\right)\left(s^{\prime}+s-4 M_{\pi}^{2}\right)},
\end{gathered}
$$

and

$$
\Delta_{0+} \equiv \operatorname{Re} F_{0+}(s)-F_{0+}\left(4 M_{\pi}^{2}\right)-\frac{s\left(s-4 M_{\pi}^{2}\right)}{\pi} \text { P.P. } \int_{4 M_{\pi}^{2}}^{\infty} d s^{\prime} \frac{\left(2 s^{\prime}-4 M_{\pi}^{2}\right) \operatorname{Im} F_{0+}\left(s^{\prime}\right)}{s^{\prime}\left(s^{\prime}-s\right)\left(s^{\prime}-4 M_{\pi}^{2}\right)\left(s^{\prime}+s-4 M_{\pi}^{2}\right)}
$$

These quantities would vanish, $\Delta_{i}=0$, if the dispersion relations were exactly satisfied.

We include in the comparison of Fig. 15 the errors; in the case of CGL, these errors are as follow from the parametrizations given by these authors in Ref. [2], for $s^{1 / 2} \lesssim 0.8 \mathrm{GeV}$. At higher energies they are taken from experiment via our parametrizations. By comparison, we show the same quantities for our best results in the present paper, that is to say, with amplitudes improved by use of dispersion relations, Eq. (4.1a) and (4.1b). In both cases we have taken the Regge parameters from Appendix B here.

\section{SUMMARY AND CONCLUSIONS}

In the previous sections we have given a representation of the $\pi \pi$ scattering amplitudes obtained fitting experimental data below $1.42 \mathrm{GeV}$, supplemented by standard Regge formulas above this energy. We have shown that our
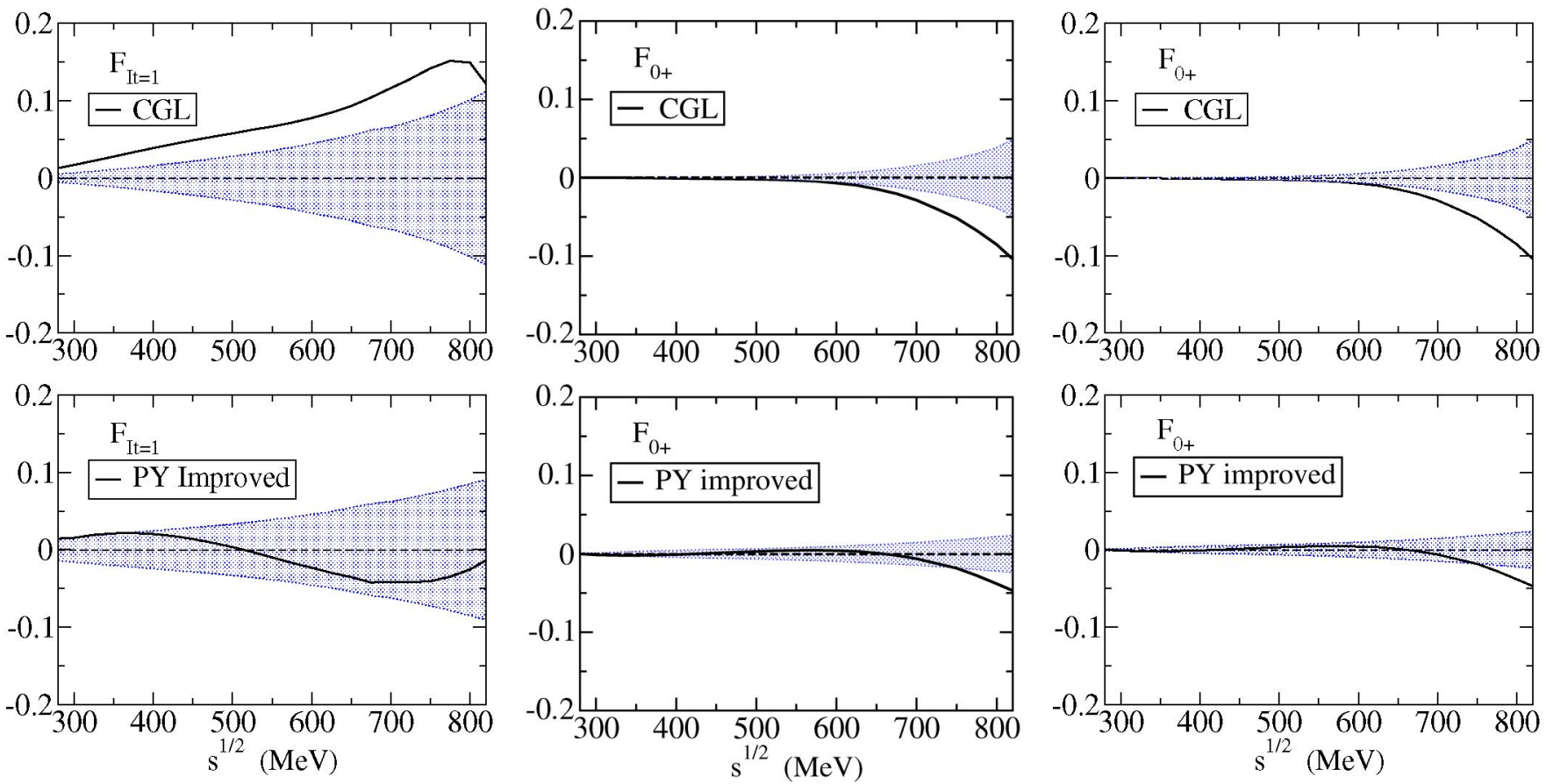

FIG. 15 (color online). Consistency of dispersion relations for the $\pi \pi$ amplitudes of Ref. [2] (CGL) and for our amplitudes, with the parameters in (4.1a) and (4.1b), denoted by PY. We plot the differences $\Delta_{i}$, given in Eqs. (7.1a)-(7.1c), between the results of the calculation of the real parts directly with the various parametrizations given, or from the dispersive formulas. Perfect consistency would occur if the continuous curves coincided with the dotted lines. The error bands are also shown. The progressive deterioration of the CGL results as the energy increases is apparent here. 


\section{J. R. PELÁEZ AND F. J. YNDURÁIN}

representations satisfy reasonably well forward dispersion relations at low energy, as well as crossing sum rules. We have shown that requiring fulfillment of the dispersion relations up to $s^{1 / 2}=0.925 \mathrm{GeV}$ leads to a refinement of the central values and errors for the parameters for the various waves, giving a set of these quantities such that dispersion relations are now satisfied, within acceptable errors, at all energies.

In particular, for the So wave, we have analyzed the results found starting from different sets of data. If we eliminate from those sets the ones that are less consistent with the dispersion relations, it is seen that the remaining determinations converge to a solution, essentially unique, when improved by requiring fulfillment of the dispersion relations. We have, therefore, obtained a complete set of $\pi \pi$ scattering amplitudes that are consistent, with theoretical requirements as well as with experiment: they are collected in Appendix A.

After this, we use these scattering amplitudes to evaluate low energy parameters for P, D0, D2, and F waves in a reliable manner, clearly improving on previous work. $[1,2,8]$ These parameters may then be used to test chiral perturbation theory to one and two loops, or to find quantities relevant for pionium decays or $C P$ violating kaon decays:

$$
\begin{aligned}
{\left[a_{0}^{(0)}-a_{0}^{(2)}\right]^{2} } & =0.077 \pm 0.008 M_{\pi}^{-2}, \\
\delta_{0}^{(0)}\left(m_{K}^{2}\right)-\delta_{0}^{(2)}\left(m_{K}^{2}\right) & =52.9 \pm 1.6^{0} .
\end{aligned}
$$

We may remark here that our errors are, typically, a factor 2 to 3 times larger than those in CGL, [2] at low energy. Given that the scattering amplitudes of this reference show mismatches between high and low energy, for many observables by several standard deviations (cf., for example, Fig. 15), and that our results are of higher precision at intermediate energies for some quantities (compare, for example, the error bars in Figs. 15), we do not consider our results to be inferior. But one certainly can ask the question whether it would be possible to improve on our precision. The answer is no, in the sense that our amplitudes agree, within errors, with theoretical requirements and with data. A sizable improvement would require substantially improved experimental data, certainly for the S0, $\mathrm{S} 2$ waves at low energy, and for the $\mathrm{S} 0$ and $\mathrm{P}$ waves above $\sim 1 \mathrm{GeV}$. One may think that imposing chiral perturbation theory could lead to decreasing the errors of the $\pi \pi$ scattering amplitudes. However, the matter is complicated and will be left for a future publication.

\section{ACKNOWLEDGMENTS}

We are grateful to CICYT, Spain, and to INTAS, for partial financial support. We are also grateful to G. Colangelo and H. Leutwyler, whose questions about the rho residue, $\beta_{\rho}(t)$, prompted us to give the alternate derivation and improvement of its values in Appendix B.
PHYSICAL REVIEW D 71, 074016 (2005)

\section{APPENDIX A: SUMMARY OF LOW ENERGY $\left(s^{1 / 2} \leq 1.42 \mathrm{GeV}\right)$ PARTIAL WAVES}

In this appendix we collect the best values for the parametrizations of the various partial waves. We give the values obtained after improving with the help of dispersion relations, Eqs. (4.1a) and (4.1b). The values of the parameters and errors obtained from raw fits to data are as given in Sec. II.

\section{The $\mathrm{S}$ wave with isospin zero below $950 \mathrm{MeV}$}

We impose the Adler zero at $s=\frac{1}{2} z_{0}^{2}$, with $z_{0}$ fixed, and a "resonance" with mass $\mu_{0}$, a free parameter. After improving the global fit with dispersion relations we have

$$
\begin{gathered}
\cot \delta_{0}^{(0)}(s)=\frac{s^{1 / 2}}{2 k} \frac{M_{\pi}^{2}}{s-\frac{1}{2} z_{0}^{2}} \frac{\mu_{0}^{2}-s}{\mu_{0}^{2}}\left\{B_{0}+B_{1} \frac{\sqrt{s}-\sqrt{s_{0}-s}}{\sqrt{s}+\sqrt{s_{0}-s}}\right\} \\
B_{0}=17.4 \pm 0.5, \quad B_{1}=4.3 \pm 1.4 \\
\mu_{0}=790 \pm 21 \mathrm{MeV} ; \quad z_{0}=195 \pm 21 \mathrm{MeV}[\text { fixed }] .
\end{gathered}
$$

This fit we take to be valid for $s^{1 / 2} \lesssim 0.95 \mathrm{GeV}$. Note that we have fixed the value of $z_{0}$ when evaluating the errors of the other parameters.

This gives

$$
\begin{aligned}
& a_{0}^{(0)}=(0.230 \pm 0.015) M_{\pi}^{-1} \\
& b_{0}^{(0)}=(0.312 \pm 0.014) M_{\pi}^{-3} \\
& \delta_{0}^{(0)}\left(m_{K}\right)=44.4^{\circ} \pm 1.5^{\circ} .
\end{aligned}
$$

\section{The $I=0 \mathrm{~S}$ wave between $950 \mathrm{MeV}$ and $1420 \mathrm{MeV}$}

We write

$$
\begin{aligned}
\cot \delta_{0}^{(0)}(s) & =c_{0} \frac{\left(s-M_{s}^{2}\right)\left(M_{f}^{2}-s\right)}{M_{f}^{2} s^{1 / 2}} \frac{\left|k_{2}\right|}{k_{2}^{2}}, \\
k_{2} & =\frac{\sqrt{s-4 m_{K}^{2}}}{2} ; \\
\eta_{0}^{(0)} & =1-\left(\epsilon_{1} \frac{k_{2}}{s^{1 / 2}}+\epsilon_{2} \frac{k_{2}^{2}}{s}\right) \frac{M^{\prime 2}-s}{s} .
\end{aligned}
$$

Then,

$$
\begin{gathered}
c_{0}=1.3 \pm 0.5, \quad M_{f}=1320 \pm 50 \mathrm{MeV} ; \\
M_{s}=920 \mathrm{MeV}\left(\text { fixed) } ; \quad \epsilon_{1}=6.4 \pm 0.5,\right. \\
\epsilon_{2}=-16.8 \pm 1.6 ; \quad M^{\prime}=1500 \mathrm{MeV} \text { (fixed). }
\end{gathered}
$$

It should be clear that this fit is not very reliable since there are several incompatible sets of experimental data. Thus, the errors are purely nominal. If using $\pi \pi \rightarrow \bar{K} K$ data to fit the inelasticity we would have obtained different values for the $\epsilon_{i}$ : 


$$
\epsilon_{1}=2.4 \pm 0.2, \quad \epsilon_{2}=-5.5 \pm 0.8 .
$$

This fit has not been improved with dispersion relations. For dispersion relations below $0.925 \mathrm{GeV}$, it is irrelevant whether we use (A5) or $\left(\mathrm{A} 5^{\prime}\right)$.

\section{Parametrization of the $S$ wave for $I=2$ below $1 \mathrm{GeV}$}

We fit only the low energy data, $s^{1 / 2}<1.0 \mathrm{GeV}$, taking the inelastic threshold at $s_{0}=1.05^{2} \mathrm{GeV}^{2}$,

$$
\begin{gathered}
\cot \delta_{0}^{(2)}(s)=\frac{s^{1 / 2}}{2 k} \frac{M_{\pi}^{2}}{s-2 z_{2}^{2}}\left\{B_{0}+B_{1} \frac{\sqrt{s}-\sqrt{s_{0}-s}}{\sqrt{s}+\sqrt{s_{0}-s}}\right\}, \\
s^{1 / 2} \leq 1.0 \mathrm{GeV} .
\end{gathered}
$$

The central values and errors are improved with dispersion relations; we get

$$
\begin{aligned}
B_{0} & =-80.8 \pm 1.7, \quad B_{1}=-77 \pm 5, \\
z_{2} & =147 \pm 7 \mathrm{MeV} \quad[\text { fixed] } ; \\
a_{0}^{(2)} & =(-0.0480 \pm 0.0046) \times M_{\pi}^{-1} ; \\
b_{0}^{(2)} & =(-0.090 \pm 0.006) \times M_{\pi}^{-3} ; \\
\delta_{0}^{(2)}\left(m_{K}^{2}\right) & =-8.5 \pm 0.3^{\circ} .
\end{aligned}
$$

\section{Parametrization of the $S$ wave for $I=2$ above $1 \mathrm{GeV}$}

At high energy, we may fit data from $s^{1 / 2}=0.91 \mathrm{GeV}$ up to $s^{1 / 2}=1.42 \mathrm{GeV}$. We require junction with the low energy fit at $1 \mathrm{GeV}$, neglect inelasticity below $1.45 \mathrm{GeV}$, and write

$$
\begin{gathered}
\cot \delta_{0}^{(2)}(s)=\frac{s^{1 / 2}}{2 k} \frac{M_{\pi}^{2}}{s-2 z_{2}^{2}}\left\{B_{0}+B_{1} \frac{\sqrt{s}-\sqrt{s_{0}-s}}{\sqrt{s}+\sqrt{s_{0}-s}}\right\} \\
s_{0}^{1 / 2}=1.45 \mathrm{GeV} ; \quad z_{2}=147 \mathrm{MeV} \text { (fixed) }
\end{gathered}
$$

After improving with dispersion relations we then find

$$
\begin{gathered}
B_{0}=-125 \pm 6, \quad B_{1}=-119 \pm 14 ; \\
s>(1.0 \mathrm{GeV})^{2} .
\end{gathered}
$$

The inelasticity may be described by the empirical fit

$$
\begin{gathered}
\eta_{0}^{(2)}(s)=1-\epsilon(1-\hat{s} / s)^{3 / 2}, \quad \epsilon=0.17 \pm 0.12 \\
\left(\hat{s}^{1 / 2}=1.05 \mathrm{GeV}\right) .
\end{gathered}
$$

These formulas are expected to hold from $1 \mathrm{GeV}$ up to $1.42 \mathrm{GeV}$.

\section{The $P$ wave below $1 \mathrm{GeV}$}

We have

$$
\begin{aligned}
\cot \delta_{1}(s) & =\frac{s^{1 / 2}}{2 k^{3}}\left(M_{\rho}^{2}-s\right)\left\{B_{0}+B_{1} \frac{\sqrt{s}-\sqrt{s_{0}-s}}{\sqrt{s}+\sqrt{s_{0}}-\bar{s}}\right\} ; \\
s_{0}^{1 / 2} & =1.05 \mathrm{GeV} .
\end{aligned}
$$

The best result, from (4.1a) and (4.1b), is

$$
\begin{gathered}
B_{0}=1.064 \pm 0.011, \quad B_{1}=0.170 \pm 0.040, \\
M_{\rho}=773.6 \pm 0.9 ; \quad a_{1}=(38.7 \pm 1.0) \times 10^{-3} M_{\pi}^{-3}, \\
b_{1}=(4.55 \pm 0.21) \times 10^{-3} M_{\pi}^{-5} .
\end{gathered}
$$

Slightly better values for $a_{1}, b_{1}$, improved with sum rules, may be found in Table IV.

\section{The $P$ wave for $1 \mathrm{GeV} \leq s^{1 / 2} \leq 1.42 \mathrm{GeV}$}

For the $\mathrm{P}$ wave between $1 \mathrm{GeV}$ and $1.4 \mathrm{GeV}$ we use an empirical formula, obtained with a linear fit to the phase and inelasticity of Protopopescu et al. [10] and Hyams et al. [11]

$$
\begin{gathered}
\delta_{1}(s)=\lambda_{0}+\lambda_{1}(\sqrt{s / \hat{s}}-1), \\
\eta_{1}(s)=1-\epsilon(\sqrt{s / \hat{s}}-1) ; \quad \epsilon=(0.30 \pm 0.15), \\
\lambda_{0}=2.69 \pm 0.01, \quad \lambda_{1}=1.1 \pm 0.2 .
\end{gathered}
$$

Here $\hat{s}=1 \mathrm{GeV}^{2}$. The value of $\lambda_{0}$ ensures the agreement of the phase with the value given by (A9a) and (A9b) at $s=\hat{s} \equiv 1 \mathrm{GeV}^{2}$. This fit is good; however, it should be remarked that there are other sets of experimental data for this wave disagreeing with the one used here for $s^{1 / 2}>$ $1.15 \mathrm{GeV}$. Hence (A10) may be biased beyond its nominal errors.

This fit has not been improved with dispersion relations.

\section{Parametrization of the $D$ wave for $I=0$}

We fit data on $\delta_{2}^{(0)}$ altogether neglecting inelasticity, which we will then add by hand. The data are scanty, and of poor quality. We impose in the fit the scattering length, as obtained from the Froissart-Gribov representation, and the experimental width of the $f_{2}$. Moreover, we improve the central values with dispersion relations.

We write

$$
\cot \delta_{2}^{(0)}(s)=\frac{s^{1 / 2}}{2 k^{5}}\left(M_{f_{2}}^{2}-s\right) M_{\pi}^{2}\left\{B_{0}+B_{1} w(s)\right\},
$$

and

$$
\begin{aligned}
& w(s)=\frac{\sqrt{s}-\sqrt{s_{0}-s}}{\sqrt{s}+\sqrt{s_{0}-s}}, \quad s_{0}^{1 / 2}=1450 \mathrm{MeV} ; \\
& M_{f_{2}}=1275.4 \mathrm{MeV} \quad \text { [fixed]. }
\end{aligned}
$$

We find,

$$
B_{0}=23.5 \pm 0.7, \quad B_{1}=24.8 \pm 1.0 .
$$


We take into account the inelasticity by writing

$\eta_{2}^{(0)}(s)= \begin{cases}1, & s<4 m_{K}^{2} ; \\ 1-\epsilon \frac{k_{2}(s)}{k_{2}\left(M_{f_{2}}^{2}\right)}, & \epsilon=0.262 \pm 0.030, s>4 m_{K}^{2} .\end{cases}$

(A11c)

Here $k_{2}=\sqrt{s / 4-m_{K}^{2}}$.

The fit returns the values

$$
\begin{aligned}
& a_{2}^{(0)}=(18.4 \pm 3.0) \times 10^{-4} \times M_{\pi}^{-5}, \\
& b_{2}^{(0)}=(-8.6 \pm 3.4) \times 10^{-4} \times M_{\pi}^{-7} .
\end{aligned}
$$

Better values for $a_{2}^{(0)}, b_{2}^{(0)}$ are given in Table IV.

\section{Parametrization of the $D$ wave for $I=2$}

For isospin equal 2, there are no resonances in the D wave. If we want a parametrization that applies down to threshold, we must incorporate the zero of the corresponding phase shift. So we write

$$
\begin{aligned}
\cot \delta_{2}^{(2)}(s)= & \frac{s^{1 / 2}}{2 k^{5}}\left\{B_{0}+B_{1} w(s)+B_{2} w(s)^{2}\right\} \\
& \times \frac{M_{\pi}^{4} s}{4\left(M_{\pi}^{2}+\Delta^{2}\right)-s}, \\
s^{1 / 2} \leq & 1.05 \mathrm{GeV} .
\end{aligned}
$$

with $\Delta$ a free parameter and

$$
w(s)=\frac{\sqrt{s}-\sqrt{s_{0}-s}}{\sqrt{s}+\sqrt{s_{0}-s}}, \quad s_{0}^{1 / 2}=1450 \mathrm{MeV} .
$$

Moreover, we impose the value for the scattering length that follows from the Froissart-Gribov representation. We assume negligible inelasticity and find, after improvement with dispersion relations,

$$
\begin{array}{ll}
B_{0}=(2.9 \pm 0.2) \times 10^{3}, & B_{1}=(7.3 \pm 0.8) \times 10^{3}, \\
B_{2}=(25.4 \pm 3.6) \times 10^{3}, & \Delta=212 \pm 19 \mathrm{MeV} .
\end{array}
$$

The fit returns reasonable values for the scattering length and effective range parameter, $b_{2}^{(2)}$ :

$$
\begin{aligned}
& a_{2}^{(2)}=(2.4 \pm 0.7) \times 10^{-4} M_{\pi}{ }^{-5} \\
& b_{2}^{(2)}=(-2.5 \pm 0.6) \times 10^{-4} M_{\pi}{ }^{-7} .
\end{aligned}
$$

Better values for $a_{2}^{(2)}, b_{2}^{(2)}$ are given in Table IV.

\section{The $\mathbf{F}$ wave}

For the $\mathrm{F}$ wave below $s^{1 / 2}=1.42 \mathrm{GeV}$ we fit the phase shifts of Protopopescu et al. [10] and those of Hyams et al. [11(b)], plus the scattering length as given by the FroissartGribov representation. After honing the central values of the parameters with the help of dispersion relations we find

$$
\begin{aligned}
\cot \delta_{3}(s) & =\frac{s^{1 / 2}}{2 k^{7}} M_{\pi}^{6}\left\{B_{0}+B_{1} \frac{\sqrt{s}-\sqrt{s_{0}-s}}{\sqrt{s}+\sqrt{s_{0}-s}}\right\} \\
B_{0} & =(1.09 \pm 0.03) \times 10^{5} \\
B_{1} & =(1.41 \pm 0.04) \times 10^{5}
\end{aligned}
$$

This implies $a_{3}=(7.0 \pm 0.8) \times 10^{-5} M_{\pi}^{-7}$, in agreement (within errors) with the result of the Froissart-Gribov calculation (Table IV).

The contribution of the $\mathrm{F}$ wave to all our sum rules is very small (but not always negligible); the interest of calculating it lies in that it provides a test (by its very smallness) of the convergence of the partial wave expansions.

\section{The $\mathbf{G}$ waves}

For the G0 wave, we take its imaginary part to be given by the tail of the $f_{4}(2050)$ resonance, with its properties as given in the particle data tables:

$$
\begin{gathered}
\operatorname{Im} \hat{f}_{4}^{(0)}(s)=\left(\frac{k(s)}{k\left(M_{f_{4}}^{2}\right)}\right)^{18} \mathrm{BR} \frac{M_{f_{4}}^{2} \Gamma^{2}}{\left(s-M_{f_{4}}^{2}\right)^{2}+M_{f_{4}}^{2} \Gamma^{2}\left[k(s) / k\left(M_{f_{4}}^{2}\right)\right]^{18}} ; \quad s^{1 / 2} \geq 1 \mathrm{GeV} ; \quad \mathrm{BR}=0.17 \pm 0.02, \\
M_{f_{4}}=2025 \pm 8 \mathrm{MeV}, \quad \Gamma=194 \pm 13 \mathrm{MeV} .
\end{gathered}
$$

For the wave G2, we can write, neglecting its eventual inelasticity,

$$
\begin{gathered}
\cot \delta_{4}^{(2)}(s)=\frac{s^{1 / 2} M_{\pi}^{8}}{2 k^{9}} B, \quad B=(-9.1 \pm 3.3) \times 10^{6} ; \\
s^{1 / 2} \geq 1 \mathrm{GeV} .
\end{gathered}
$$

It should be noted that the expressions for the G0, G2 waves, are little more than order of magnitude estimates.
Moreover, at low energies they certainly fail; below $1 \mathrm{GeV}$, expressions in terms of the scattering length approximation, with

$$
\begin{aligned}
& a_{4}^{(0)}=(8.0 \pm 0.4) \times 10^{-6} M_{\pi}^{-9}, \\
& a_{4}^{(2)}=(4.5 \pm 0.2) \times 10^{-6} M_{\pi}^{-9},
\end{aligned}
$$

are more appropriate. If, in a given calculation, the contribution of either of the $2 \mathrm{G}$ waves is important, it means 
that the calculation will have a large error. We have checked in a representative number of our calculations that the contributions of the G0, G2 waves are totally negligible.

\section{APPENDIX B: THE REGGE AMPLITUDE $\left(s^{1 / 2} \geq 1.42 \mathrm{GeV}\right)$}

In the calculations of the various sum rules and dispersion relations we require the imaginary part of the scattering amplitudes at high energy. For this we use Regge formulas, applicable when $s \gg \Lambda^{2}$, with $\Lambda \simeq 0.3 \mathrm{GeV}$ the QCD parameter, and $s \gg|t|$. Specifically, we use them above $s^{1 / 2}=1.42 \mathrm{GeV}$ and expect them to be valid at least for $|t| \lesssim 4 M_{\pi}^{2} \simeq 0.08 \mathrm{GeV}^{2}$. These formulas are obtained from $N N, \pi N$ scattering, using factorization, and from direct fits to $\pi \pi$ data. We will not include refinements that take into account the logarithmic increase of total cross sections at ultrahigh energies (that may be found in Ref. [5]), superfluous for applications to $\pi \pi$ scattering. We give here the relevant formulas, for ease of reference, for Pomeron and $P^{\prime}$ Regge poles, and will only discuss in some detail (and even improve slightly) the parameters of the rho residue.

We consider three Regge poles: Pomeron $(P)$, the $P^{\prime}$ [associated with the $f_{2}(1270)$ resonance] and the rho. For the first two,

$$
\begin{aligned}
\operatorname{Im} F_{\pi \pi}^{\left(I_{t}=0\right)}(s, t) \underset{\substack{s \rightarrow \infty \\
t \text { fixed }}}{\simeq} P(s, t)+P^{\prime}(s, t), \\
P(s, t)=\beta_{P} \alpha_{P}(t) \frac{1+\alpha_{P}(t)}{2} e^{b t}(s / \hat{s})^{\alpha_{P}(t)}, \\
P^{\prime}(s, t)=\beta_{P^{\prime}} \frac{\alpha_{P^{\prime}}(t)\left[1+\alpha_{P^{\prime}}(t)\right]}{\alpha_{P^{\prime}}(0)\left[1+\alpha_{P^{\prime}}(0)\right]} e^{b t}(s / \hat{s})^{\alpha_{P^{\prime}}(t)}, \\
\alpha_{P^{\prime}}(t)=\alpha_{\rho}(t) .
\end{aligned}
$$

For exchange of unit isospin, we write

$$
\operatorname{Im} F_{\pi \pi}^{\left(I_{t}=1\right)}(s, t) \underset{\substack{s \rightarrow \infty \\ t \text { fixed }}}{\simeq} \rho(s, t) ; \quad \rho(s, t)=\beta_{\rho}(t)(s / \hat{s})^{\alpha_{\rho}(t)} .
$$

In the present paper we have taken a formula slightly different from that in Ref. [5], viz.,

$$
\begin{aligned}
\beta_{\rho}(t) & =\beta_{\rho}(0) \frac{1+\alpha_{\rho}(t)}{1+\alpha_{\rho}(0)}\left[1+d_{\rho} t\right] e^{b t} \underset{t \rightarrow 0}{\simeq} \beta_{\rho}(0)\left[1+c_{\rho} t\right] \\
c_{\rho} & =b+d_{\rho}+\frac{\alpha_{\rho}^{\prime}(0)}{1+\alpha_{\rho}(0)}
\end{aligned}
$$

In Ref. [5], where we took an expression from Rarita et al. [18], solution 1a, assuming the $t$ dependence to be equal for $\pi \pi$ and $\pi N$ scattering [see below, Eq. (B7)]. Our expression now, (B2b), is simpler. As we will show below, we can fix its parameters (for small values of $t$ ) without assuming the equality of $\pi \pi$ and $\pi N$.

For exchange of isospin two,

$$
\operatorname{Im} F_{\pi \pi}^{\left(I_{t}=2\right)}(s, t) \underset{\substack{s \rightarrow \infty \\ t \text { fixed }}}{\simeq} R_{2}(s, t) \equiv \beta_{2} e^{b t}(s / \hat{s})^{\alpha_{\rho}(t)+\alpha_{\rho}(0)-1} .
$$

This last amplitude corresponds to double rho exchange; the expression we use for it at $t \neq 0$ is rather arbitrary, since little is known about it. Fortunately, it has almost no influence in the calculations.

Following the analysis of Ref. [5], the trajectories are taken as follows:

$\alpha_{P}(t) \underset{t \sim 0}{\simeq} \alpha_{P}(0)+\alpha_{P}^{\prime} t, \quad \alpha_{\rho}(t) \underset{t \sim 0}{\simeq} \alpha_{\rho}(0)+\alpha_{\rho}^{\prime} t+\frac{1}{2} \alpha_{\rho}^{\prime \prime} t^{2}$,

and one has

$$
\begin{gathered}
\alpha_{\rho}(0)=0.52 \pm 0.02, \quad \alpha_{\rho}^{\prime}=0.90 \mathrm{GeV}^{-2}, \\
\alpha_{\rho}^{\prime \prime}=-0.3 \mathrm{GeV}^{-4} ; \quad \alpha_{P}(0)=1, \\
\alpha_{P}^{\prime}=(0.20 \pm 0.10) \mathrm{GeV}^{-2} .
\end{gathered}
$$

Moreover, in the present paper, we have taken the following values for the parameters of the residues:

$$
\begin{gathered}
\beta_{P}=2.54 \pm 0.03, \quad \beta_{P^{\prime}}=1.05 \pm 0.02, \\
\beta_{2}=0.2 \pm 0.2, \quad b=(2.4 \pm 0.2) \mathrm{GeV}^{-2} ; \\
\beta_{\rho} \equiv \beta_{\rho}(0)=1.02 \pm 0.11, \quad d_{\rho}=(2.4 \pm 0.9) \mathrm{GeV}^{-2} .
\end{gathered}
$$

The parameters for $P, P^{\prime}$ are like in Ref. [5]. Those for the rho trajectory are as improved below.

The Pomeron and $P^{\prime}$ parameters are well fixed from factorization and (besides $\pi \pi$ cross sections) by $\pi N, N N$ data, down to kinetic energies $\simeq 1 \mathrm{GeV}$; but some extra words will be added on the rho residue, $\beta_{\rho}(t)$. This quantity was obtained in Ref. [5] in a mixed manner: $\beta_{\rho}=$ $\beta_{\rho}(0)$ was found fitting high energy $\pi \pi$ data, improving the error with the help of the sum rule (5.2). The $t$ dependence of $\beta_{\rho}(t)$ was taken as in solution 1a of Rarita et al. [18] assuming it to be identical for $\pi \pi$ and $\pi N$ scattering. Thus, we wrote

$$
\begin{aligned}
\beta_{\rho}(t)= & \beta_{\rho}(0)\left[(1.5+1) e^{b t}-1.5\right] \\
& \times \frac{1+\alpha_{\rho}(t)}{1+\alpha_{\rho}(0)} \underset{t \rightarrow 0}{\simeq} \beta_{\rho}(0)\left[1+c_{\rho} t\right], \\
c_{\rho}= & 2.5 b+\frac{\alpha_{\rho}^{\prime}(0)}{1+\alpha_{\rho}(0)} .
\end{aligned}
$$

This, in fact, is similar to (B2b) and (B6) for small values of $|t|$, as we will check below.

There are other possibilities for the $t$ dependence of the rho residue. We could have chosen solution 1 of Rarita $e t$ 
al. [18] or we could have taken a Veneziano-type formula with a diffractive factor included, writing

$$
\begin{aligned}
\rho(s, t)= & \beta_{\rho}(t)(s / \hat{s})^{\alpha_{\rho}(t)} ; \\
\beta_{\rho}(t)= & \beta_{\rho}(0) \frac{\Gamma\left[1-\alpha_{\rho}(t)\right] \sin \pi \alpha_{\rho}(t)}{\Gamma\left[1-\alpha_{\rho}(0)\right] \sin \pi \alpha_{\rho}(0)} \\
& \times e^{b t} \underset{t \rightarrow 0}{\simeq} \beta_{\rho}(0)\left[1+c_{\rho} t\right], \\
c_{\rho} \simeq & b+\gamma_{\mathrm{E}} \alpha_{\rho}^{\prime}(0) .
\end{aligned}
$$

Another possibility is afforded by the $t$ dependence obtained by Froggatt and Petersen [19] from an analysis of $\pi^{+} \pi^{-}$dispersion relations at fixed $t$. These authors take

$$
\beta_{\rho}(t)=\beta_{\rho}(0) \frac{\sin \pi \alpha_{\rho}(t)}{\sin \pi \alpha_{\rho}(0)}\left[1-t / t_{\rho}\right] e^{b_{\mathrm{FP}} t},
$$

with $t_{\rho}^{-1}=-2.2 \mathrm{GeV}^{-2}, b_{\mathrm{FP}}=0.8 \mathrm{GeV}^{-2}$. This gives a slope for the residue of about half the value of solution $1 \mathrm{a}$ of Rarita et al.

The values of $\beta_{\rho}(t)$, both $\beta_{\rho}(0)$ and $t$ dependence, are given by Regge fits with less precision than other quantities; a few determinations (including the ones mentioned here) are shown in Fig. 16. The variation from one estimate to another in the range necessary for Roy equations, $-t \simeq$ $0.6 \mathrm{GeV}^{2}$, is very large, and even at $|t| \simeq 0.1 \mathrm{GeV}^{2}$ there are noticeable differences.

Fortunately, we can at least check the correctness (within errors) of the values of $\beta_{\rho}(t)$ given in Ref. [5] at $t=0$ and $t=4 M_{\pi}^{2} \simeq 0.08 \mathrm{GeV}^{2}$, and even improve them. In fact, apart from the sum rule (5.2), the fulfillment of the dispersion relation (3.5) fixes with good accuracy $\beta_{\rho}(0)$, and combinations of both values $\beta_{\rho}(0)$ and $\beta_{\rho}\left(4 M_{\pi}^{2}\right)$ enter in the evaluations, using the FroissartGribov representation, of two quantities, $a_{1}, b_{1}$, that can also be determined by other methods, independent of the Regge assumptions. The ensuing equality of the two estimates each for $a_{1}, b_{1}$ given in Eqs. (6.2) and (6.4) fixes $c_{\rho}$, as we will see below. Thus, if, for example, we had taken the Veneziano-type $t$ dependence of Palou and Ynduráin [19] [i.e., as in (B8) without the exponential factor $e^{b t}$ ) we would have obtained $b_{1} \sim 3 \times 10^{-3} M_{\pi}^{-5}$, quite incompatible with determinations from the pion form factor or the sum rule (6.8). Even if we include a diffraction factor as in (B8), the values of $a_{1}, b_{1}$ following from the Froissart-Gribov representation vary by more than $1 \sigma$.

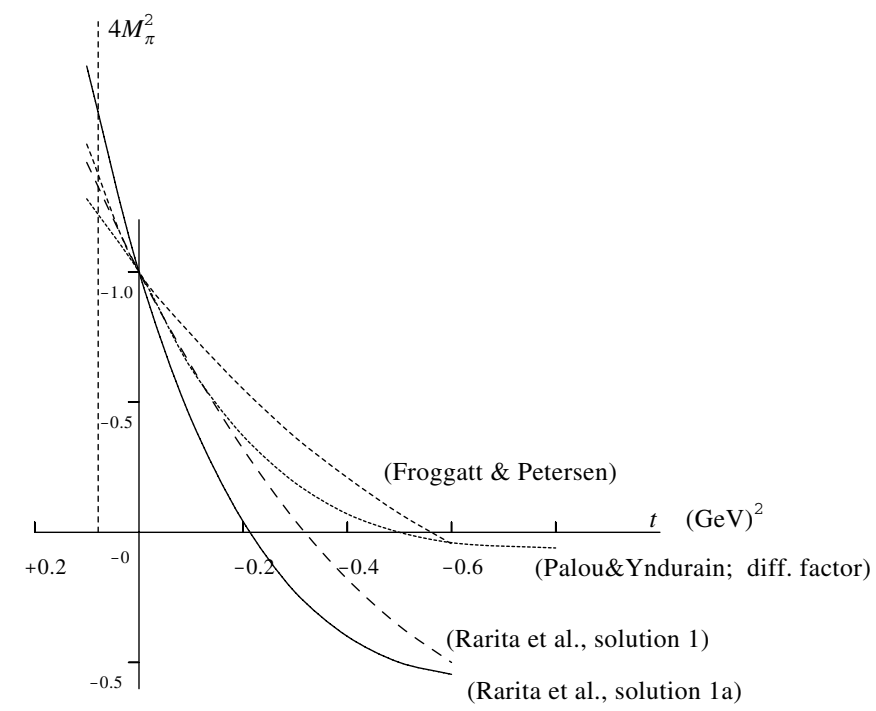

FIG. 16. The $t$ dependence of the different Regge residues, $\beta(t) / \beta(0)$ (Refs. $[18,19]$ ): Rarita et al., solution 1 and solution 1a: the fits from Rarita et al. (assuming equal slope for $\pi N$ and $\pi \pi)$. Froggatt and Petersen: from fits to $\pi \pi$ dispersion relations at fixed $t$. Palou and Yndurain; diffraction factor: actually, from the Veneziano model, including a diffraction factor $e^{b t}, b=$ $2.4 \mathrm{GeV}$, as in Eq. (B7). The residue given in (B6) would lie between "Palou and Yndurain diffraction factor" and "Rarita et al. solution 1a" (closer to the second).

One can in principle fix the parameters for $\pi \pi$ scattering from other processes profiting from factorization, which allows one to improve the poor precision of the $\pi \pi$ data. This is all right for the $P, P^{\prime}$ poles because here we can use data from $\pi N$ and $\bar{p} p+p p$, scattering which are very precise and to which only these two poles, plus the rho pole for $\pi N$, contribute. The situation is different for the coupling of the rho pole to $\pi \pi$. Direct fits to $\pi \pi$ data are not precise and, to use factorization, one has to incorporate (besides $\pi N$ ) proton, antiproton and neutron scattering data. The last presents important systematic errors because the data have to be extracted from scattering on deuterons. The large number of trajectories that contribute to these processes also make the analysis unreliable; for example, some existing fits have very likely their rho trajectory contaminated by pion exchange, which is not negligible at the lower energy range. Because of this, we will now give a new determination of $\beta_{\rho}(0)$ and $d_{\rho}$ independent of Regge fits.

To obtain $\beta_{\rho}(0)$, we rewrite the dispersion relation (3.7) as

$\operatorname{Re} F^{\left(I_{t}=1\right)}(s, 0)-\frac{2 s-4 M_{\pi}^{2}}{\pi}$ P.P. $\int_{4 M_{\pi}^{2}}^{1.42^{2} \mathrm{GeV}^{2}} d s^{\prime} \frac{\operatorname{Im} F^{\left(I_{t}=1\right)}\left(s^{\prime}, 0\right)}{\left(s^{\prime}-s\right)\left(s^{\prime}+s-4 M_{\pi}^{2}\right)}=\frac{2 s-4 M_{\pi}^{2}}{\pi} \int_{1.42^{2} \mathrm{GeV}^{2}}^{\infty} d s^{\prime} \frac{\operatorname{Im} F^{\left(I_{t}=1\right)}\left(s^{\prime}, 0\right)}{\left(s^{\prime}-s\right)\left(s^{\prime}+s-4 M_{\pi}^{2}\right)}$

We evaluate the left hand side for values of $s^{1 / 2}$ from threshold to $925 \mathrm{MeV}$, at intervals of $25 \mathrm{MeV}$, using low energy 
experimental data, via the parametrizations ${ }^{6}$ given in Sec. II. In this region, $s^{1 / 2} \leq 0.925 \mathrm{GeV}$, the uncertainties due to the middle energy S0 and P waves are not very important. If we write in the right hand side of (B10)

$$
\operatorname{Im} F^{\left(I_{t}=1\right)}\left(s^{\prime}, 0\right)=\beta_{\rho}(0)\left(s^{\prime} / \hat{s}\right)^{\alpha_{\rho}(0)}, \quad s \geq 1.42^{2} \mathrm{GeV}^{2},
$$

and fix $\alpha_{\rho}(0)=0.52$ from factorization or deep inelastic scattering, we may fit $\beta_{\rho}(0)$ and find a very precise number,

$$
\beta_{\rho}(0)=1.06 \pm 0.10
$$

This is quite compatible with the value $\beta_{\rho}(0)=0.94 \pm 0.14$ that we obtained from fits to $\pi \pi$ cross sections in Ref. [5]. The result (B11) is robust; if we double the errors of the parameters for the $\mathrm{P}$ and $\mathrm{S} 0$ waves between 1 and $1.42 \mathrm{GeV}$, where (as discussed in the main text) one has larger uncertainties, and repeat the fit, we get the same value.

As for $c_{\rho}$, we can use the values of $a_{1}, b_{1}$ obtained from the analysis of the pion form factor [9], together with the Froissart-Gribov representation of the same quantities. We rewrite Eq. (6.1) as

$$
a_{1}-\frac{\sqrt{\pi} \Gamma(2)}{4 M_{\pi} \Gamma(1+3 / 2)} \int_{4 M_{\pi}^{2}}^{1.42^{2} \mathrm{GeV}^{2}} d s \frac{\operatorname{Im} F^{\left(I_{t}=1\right)}\left(s, 4 M_{\pi}^{2}\right)}{s^{3}}=\frac{\sqrt{\pi} \Gamma(2)}{8 M_{\pi} \Gamma(1+3 / 2)} \int_{1.42^{2} \mathrm{GeV}^{2}}^{\infty} d s \frac{\operatorname{Im} F^{\left(I_{t}=1\right)}\left(s, 4 M_{\pi}^{2}\right)}{s^{3}} .
$$

For the $b_{l}, l=$ odd,

$$
\begin{aligned}
& b_{l}-\frac{\sqrt{\pi} \Gamma(l+1)}{4 M_{\pi} \Gamma(l+3 / 2)} \int_{4 M_{\pi}^{2}}^{1.42^{2} \mathrm{GeV}^{2}} d s\left\{\frac{4 \operatorname{Im} F^{\left(I_{t}=1\right)^{\prime}}\left(s, 4 M_{\pi}^{2}\right)}{\left(s-4 M_{\pi}^{2}\right) s^{l+1}}-\frac{(l+1) \operatorname{Im} F\left(s, 4 M_{\pi}^{2}\right)}{s^{l+2}}\right\} \\
& =\frac{\sqrt{\pi} \Gamma(l+1)}{4 M_{\pi} \Gamma(l+3 / 2)} \int_{1.42^{2} \mathrm{GeV}^{2}}^{\infty} d s\left\{\frac{4 \operatorname{Im} F^{\left(I_{t}=1\right)^{\prime}}\left(s, 4 M_{\pi}^{2}\right)}{\left(s-4 M_{\pi}^{2}\right) s^{l+1}}-\frac{(l+1) \operatorname{Im} F\left(s, 4 M_{\pi}^{2}\right)}{s^{l+2}}\right\} ; \\
& \operatorname{Im} F^{\left(I_{t}=1\right)^{\prime}}(s, t) \equiv \partial \operatorname{Im} F^{\left(I_{t}=1\right)}(s, t) / \partial \cos \theta .
\end{aligned}
$$

The left hand sides in (B12a) and (B12b) are evaluated using also the experimental phase shifts and inelasticities as fitted in Sec. II of the present paper and we take, in the right hand side of (B12a),

$$
\begin{aligned}
\operatorname{Im} F^{\left(I_{t}=1\right)}\left(s, 4 M_{\pi}^{2}\right) & =\beta_{\rho}\left(4 M_{\pi}^{2}\right)(s / \hat{s})^{\alpha_{\rho}\left(4 M_{\pi}^{2}\right),} \\
s & \geq 1.42^{2} \mathrm{GeV}^{2},
\end{aligned}
$$

$\beta_{\rho}(t) \simeq \beta_{\rho}(0)\left[1+c_{\rho} t\right]$. An analogous formula may be written for the right hand side of $(\mathrm{B} 12 \mathrm{~b})$. The sum rule for $b_{1}$ gives the more precise constraint for $c_{\rho}$ for two reasons. First, it depends directly on the derivative of $\operatorname{Im} F^{\left(I_{t}=1\right)}(s, t)$ with respect to $t(\cos \theta)$, hence $c_{\rho}$ appears explicitly; and, second, the low energy contributions to the left hand side in (B12b) cancel to a large extent, so $b_{1}$ is given almost exclusively by the high energy, Regge formulas; cf. Ref. [6].

\footnotetext{
${ }^{6}$ Of course, before improving with dispersion relations, or the reasoning would be somewhat circular. Alternatively, we could fit simultaneously the parameters for phase shifts and inelasticities and the Regge parameters of the rho, using all three dispersion relations; the method, quite complicated, would not give results substantially different from what we get making independent fits.
}

We can fit, with these sum rules for $a_{1}, b_{1}$, plus the dispersion relation for $I_{t}=1$, the parameters $\beta_{\rho}(0), c_{\rho}$ simultaneously. As was to be expected, the value of $\beta_{\rho}(0)$ is unchanged and, for $c_{\rho}$, we find the values

$$
c_{\rho}= \begin{cases}(6.0 \pm 2.8) \mathrm{GeV}^{-2} & \text { from } a_{1} \\ (4.7 \pm 0.8) \mathrm{GeV}^{-2} & \text { from } b_{1}\end{cases}
$$

This is compatible with what one has from solution 1a of Rarita et al. [18], $c_{\rho}=(6.6 \pm 0.5) \mathrm{GeV}^{-2}$ in the sense that they produce similar values for $\beta_{\rho}\left(4 M_{\pi}^{2}\right)$ : the decrease in $c_{\rho}$ is compensated (within errors) by the increase in $\beta_{\rho}(0)$, Eq. (B1), compared with what we used in Ref. [5]. Our results for $c_{\rho}$ thus justify the use of the formulas of solution 1a of Rarita et al. [18], for small values of $|t| \lesssim 0.1 \mathrm{GeV}$, as was done in Ref. [6], and the assumption that (in that range) they are also valid for $\pi \pi$ scattering, within errors.

We can also include in the fit the information from $\pi \pi$ scattering data (as discussed in Ref. [5]), since it is compatible with what we found now, and the sum rule (5.2), evaluated with the parameters found in Sec. II here for the low energy piece. In this case we find what we consider the best results, 


$$
\beta_{\rho}(0)=1.02 \pm 0.11, \quad c_{\rho}=(5.4 \pm 0.9) \mathrm{GeV}^{-2} .
$$

This implies

$$
d_{\rho}=(2.4 \pm 0.9) \mathrm{GeV}^{-2} .
$$

Equations (B14a) and (B14b) provide us with the central values given in (B6) for $d_{\rho}, \beta_{\rho}(0)$.

\section{APPENDIX C: ON EXPERIMENTAL PHASE SHIFTS IN THE RANGE $1.4 \mathrm{GeV} \simeq s^{1 / 2} \simeq 2 \mathrm{GeV}$}

As is well known, as soon as the center of mass kinetic energy in a hadronic reaction, $E_{\text {kin,c.m. }}$, increases beyond $1 \mathrm{GeV}$, inelastic processes become important and, for $E_{\text {kin,c.m. }} \gtrsim 1.3 \mathrm{GeV}$, they dominate elastic ones. This is easily understandable in the QCD, ladder version of the Regge picture, as discussed in Ref. [6]; and indeed, it is verified experimentally in the hadronic processes $\pi N, K N$, and $N N$ where, for $E_{\text {kin,c.m. }}>1.3 \mathrm{GeV}$, the elastic cross section is smaller than the inelastic one and, for $E_{\text {kin,c.m. }}>$ $1.5 \mathrm{GeV}$, the elastic cross section is less than or about one half of the inelastic one. There is no reason to imagine that $\pi \pi$ scattering would follow a different pattern. In this case (large inelasticity), and again as mentioned in Refs. [6,7], it can be proved theoretically [20] that there is not a unique solution to the phase shift analysis: some sets of $\eta$ s and $\delta \mathrm{s}$ may fit the data; but so would others.

In spite of this, the Cern-Munich experiments ${ }^{7}$ have produced a set of phase shifts and inelasticities which go up to $s^{1 / 2} \simeq 2 \mathrm{GeV}$. These have been used as input in certain theoretical analyses, notably in those of Refs. [1,2]. Unfortunately, such phase shifts and inelasticities are very likely to diverge more and more from reality as $s^{1 / 2}$ becomes larger and larger than $1.3 \mathrm{GeV} \simeq 2 M_{\pi}+$ $1 \mathrm{GeV}$. This is suggested, besides the theoretical reasons just mentioned, by the fact that the Cern-Munich phase shifts and inelasticities contradict a number of physical properties they should fulfill: we will here mention a few.

As we have remarked above, one would expect dominant inelastic cross sections above $s^{1 / 2} \sim 1.5 \mathrm{GeV}$; but the Cern-Munich elastic cross sections are larger or comparable to the inelastic ones up to $s^{1 / 2}=2 \mathrm{GeV}$. What is worse, the $\pi^{+} \pi^{-}$inelastic cross section of Cern-Munich, alone of all hadronic cross sections, decreases as the kinetic energy increases between 1 and $1.7 \mathrm{GeV}$, as shown, for example, in Fig. 7 in the paper of Hyams et al. [11(a)]. As happens for $\gamma N, \pi N, K N$, or $N N$ scattering, and indeed as is also seen in other experimental analyses for $\pi \pi$ scattering [4], we expect a leveling off of the total cross section for $E_{\text {kin,c.m. }}>1.3 \mathrm{GeV}$ : but the Cern-Munich total cross section for $\pi^{+} \pi^{-}$scattering decreases roughly like

\footnotetext{
${ }^{7}$ Hyams et al. and Grayer et al., Refs. [11(a),11(b)].
}

$1 / s$ up to $2 \mathrm{GeV}$. In fact, the Cern-Munich elastic cross section agrees well with that found in other experiments (such as those in Ref. [4]), but their total cross section is smaller by a factor $\sim 2$ than those in Refs. [4], at the higher part of the energy range: the Cern-Munich results certainly misrepresent the total cross section. From all this it follows that the Cern-Munich phases and inelasticities are not reliable in that energy region.

Second, the combination of $\delta$ and $\eta$ for both $\mathrm{P}$ and $\mathrm{S} 0$ waves at energy $\gtrsim 1.8 \mathrm{GeV}$ is incompatible with QCD results for the electromagnetic form factor of the pion, and also with Regge behavior, which requires all phases to go to a multiple of $\pi$ at high energy, and the real part of the partial wave amplitudes for isospin 0,1 , to be positive. The phase $\delta_{1}$ is also incompatible with QCD results for the electromagnetic form factor of the pion. In fact, the phase of this form factor behaves like

$$
\delta_{\text {form factor }}(s) \simeq \pi\left(1+\frac{1}{\log s / \hat{t}}\right), \quad s \gg \Lambda^{2} ; \quad \hat{t} \sim \Lambda^{2} ;
$$

$\Lambda$ is the QCD parameter (see, e.g., Ref. [21]). One may take this to hold for $s \geqslant 3 \mathrm{GeV}^{2}\left(s^{1 / 2}>1.6 \mathrm{GeV}\right)$. If one had negligible inelasticity for the $\mathrm{P}$ wave somewhere in the region $1.6 \mathrm{GeV} \lesssim s^{1 / 2} \lesssim 2 \mathrm{GeV}$, as the Cern-Munich data seem to imply, the form factor and partial wave would have the same phase at such energies, and thus the same behavior should hold for $\delta_{1}(s), \delta_{\text {form factor }}(s)$. But the phase which the Cern-Munich experiment gives clearly contradict this behavior around $1.8 \mathrm{GeV}$; there the Cern-Munich phase $\delta_{1}$ stays consistently below $\pi$, while, as just shown, it should be above.

Third, for the process $\pi^{ \pm} \pi^{ \pm} \rightarrow \pi^{ \pm} \pi^{ \pm}$, we would expect large inelasticity as soon as the production $\pi^{ \pm} \pi^{ \pm} \rightarrow$ $\rho^{ \pm} \rho^{ \pm}$is energetically possible, and therefore large inelasticity for the S2, D2 waves for $s^{1 / 2} \gg 2 M_{\rho}$. This, in particular, occurs in any theoretical model. It is therefore not possible to extrapolate these phases above $\sim 1.45 \mathrm{GeV}$ as being elastic. In fact, the extrapolation used in Refs. [1,2] for the D2 wave is clearly incorrect above $\sim 1.4 \mathrm{GeV}$ as the corresponding $\left|\delta_{2}^{(2)}(s)\right|$ grows linearly with $s$ while, from Regge theory (and also from low energy fits, see Fig. 7), one expects it to go to zero. Thus, besides the general problem for the cross sections we have individual problems for each of the S0, S2, P, and D2 phases.

Finally we would like to mention that both the Regge picture and the values of the experimental cross sections for all hadronic processes indicate that the number of waves that contribute effectively to the imaginary part (say) of the scattering amplitudes grows with the kinetic energy as $E_{\text {kin }} / \Lambda$, for $E_{\text {kin }}$ upwards of $1 \mathrm{GeV}$. We thus expect 2 to 3 waves (for fixed isospin) at $E_{\text {kin }} \sim 1 \mathrm{GeV}$, and almost double this, 4 or 5 waves, at $E_{\text {kin }} \sim 1.7 \mathrm{GeV}$. In fact, for $\pi \pi$ scattering at this energy, the contribution to the total cross section of the $\mathrm{F}$ wave is as large as that of the $\mathrm{P}$ 
wave, the D0 wave is as large as the S0 wave and the contribution of the D2 wave is actually larger than that of the S2 wave. The partial wave series with only two waves per isospin channel is not convergent, and the approximations, like those of Refs. $[1,2,11]$, that neglect higher waves at such energies have yet another reason for being unrealistic [14].

Because of all this, it follows that use of the CernMunich phases and inelasticities must lead to a rather distorted imaginary part of the $\pi \pi$ scattering amplitude above $s^{1 / 2} \sim 1.4 \mathrm{GeV}$. It is thus not surprising that authors like those in Refs. [1,2,15], who fix their Regge parameters by balancing them above $2 \mathrm{GeV}$ with Cern-Munich phase shift analyses below $2 \mathrm{GeV}$, get incorrect values for the first. $^{8}$

\footnotetext{
${ }^{8}$ We would like to emphasize that what has been said should not be taken as implying criticism of the Cern-Munich experiment which, for $s^{1 / 2} \lesssim 1.4 \mathrm{GeV}$, produced what are probably the best determinations of phase shifts and inelasticities. Above $1.4 \mathrm{GeV}$, they did what they could: it is for theorists to realize that this was not enough to produce acceptable phase shifts and inelasticities at these higher energies.
}

[1] B. Ananthanarayan et al., Phys. Rep. 353, 207 (2001).

[2] G. Colangelo, J. Gasser, and H. Leutwyler, Nucl. Phys. B603, 125 (2001).

[3] S. M. Roy, Phys. Lett.36B, 353 (1971).

[4] N. N. Biswas et al., Phys. Rev. Lett. 18, 273 (1967) $\left[\pi^{-} \pi^{-}, \pi^{+} \pi^{-}\right.$, and $\pi^{0} \pi^{-}$]; D. Cohen et al., Phys. Rev. D 7, 661 (1973) [ $\pi^{-} \pi^{-}$]; W. J. Robertson, W. D. Walker, and J. L. Davis, Phys. Rev. D 7, 2554 (1973) [ $\left.\pi^{+} \pi^{-}\right]$; W. Hoogland et al., Nucl. Phys. B126, 109 (1977) $\left[\pi^{-} \pi^{-}\right]$; J. Hanlon et al., Phys. Rev. Lett. 37, 967 (1976) $\left[\pi^{+} \pi^{-}\right]$; H. Abramowicz et al., Nucl. Phys. B166, 62 (1980) [ $\left.\pi^{+} \pi^{-}\right]$. These references cover the region between 1.35 and $16 \mathrm{GeV}$, and agree within errors in the regions where they overlap (with the exception of $\pi^{-} \pi^{-}$below $2.3 \mathrm{GeV}$, discussion in Ref. [5]).

[5] J. R. Peláez and F. J. Ynduráin, Phys. Rev. D 69, 114001 (2004).

[6] J. R. Peláez and F. J. Ynduráin, Phys. Rev. D 68, 074005 (2003).

[7] F. J. Ynduráin, hep-ph/0310206.

[8] S. Descotes, N. H. Fuchs, L. Girlanda, and J. Stern, Eur. Phys. J. C, 24, 469 (2002); R. Kamiński, L. Leśniak, and B. Loiseau, Phys. Lett. B 551, 241 (2003).

[9] J.F. de Trocóniz and F. J. Ynduráin, Phys. Rev. D 65, 093001 (2002); hep-ph/0402285 [Phys. Rev. D (to be published)]. When quoting numbers, we will quote from this last paper.

[10] S. D. Protopopescu et al., Phys. Rev. D 7, 1279 (1973).

[11] The Cern-Munich experiments ( $\pi \pi$ scattering). (a) B. Hyams, Nucl. Phys. B64, 134 (1973); G. Grayer et al., Nucl. Phys. B75, 189 (1974); see also the analysis of the same experimental data in P. Estabrooks and A. D. Martin, Nucl. Phys. B79, 301 (1974). (b) B. Hyams et al., Nucl. Phys. B100, 205 (1975). (c) R. Kamiński, L. Lesniak, and K. Rybicki, Z. Phys. C 74, 79 (1997); Eur. Phys. J. direct C 4, 4 (2002). $\pi \pi \rightarrow \bar{K} K$ scattering: (d) W. Wetzel et al., Nucl. Phys. B115, 208 (1976); V. A. Polychromatos et al., Phys. Rev. D 19, 1317 (1979); D. Cohen et al., Phys. Rev. D 22, 2595 (1980); E. Etkin et al., Phys. Rev. D 25, 1786 (1982).

[12] M. J. Losty et al., Nucl. Phys. B69, 185 (1974); W. Hoogland et al., Nucl. Phys. B126, 109 (1977); N. B. Durusoy et al., Phys. Lett. B 45, 517 (1973).

[13] L. Rosselet et al., Phys. Rev. D 15, 574 (1977); S. Pislak et al., Phys. Rev. Lett. 87, 221801 (2001).

[14] For the more recent determination, see A. Aloisio et al., Phys. Lett. B 538, 21 (2002); an older one is P. Pascual and F. J. Ynduráin, Nucl. Phys. B83, 362, (1974).

[15] M. R. Pennington, Ann. Phys. (N.Y.) 92, 164 (1975).

[16] B. R. Martin, D. Morgan, and G. Shaw, Pion-Pion Interactions in Particle Physics (Academic Press, New York, 1976).

[17] F. P. Palou, J.L. Sánchez-Gómez, and F. J. Ynduráin, Z. Phys. A 274, 161 (1975).

[18] W. Rarita et al., Phys. Rev. 165, 1615 (1968).

[19] C. D. Froggatt and J. L. Petersen, Nucl. Phys. B129, 89 (1977); F. P. Palou and F. J. Ynduráin, Nuovo Cimento 19A, 245 (1974).

[20] D. Atkinson et al., Nucl. Phys. B7, 375 (1968); Nucl. Phys. B23, 397 ( 1970).

[21] F. J. Ynduráin, Phys. Lett. B 578, 99 (2004); Phys. Lett. B 586, 439(E) (2004); hep-ph/0501104 [Phys. Lett. B (to be published)]. 